cond-mat/9411022

\title{
Lectures on Directed Paths in Random Media
}

\author{
by \\ Mehran Kardar \\ Department of Physics \\ Massachusetts Institute of Technology \\ Cambridge, Massachusetts 02139, USA
}

Presented at:

\section{Les Houches Summer School}

on

Fluctuating Geometries in

Statistical Mechanics and Field Theory

August 1994 


\section{Table of Contents}

A. Introduction . . . . . . . . . . . . . . . . . . . . . . . . . . . . 2

B. High Temperature Expansions for the Ising Model . . . . . . . . . . . . . . 囵

C. Characteristic Functions and Cumulants . . . . . . . . . . . 6

D. The One Dimensional Chain . . . . . . . . . . . . . . . . . . . . . . . 9

E. Directed Paths and the Transfer Matrix . . . . . . . . . . . . . . . 13

F. Moments of the Correlation Function . . . . . . . . . . . . . . . . . . . 18

G. The Probability Distribution in Two Dimensions . . . . . . . . . . . . . 22

H. Higher Dimensions . . . . . . . . . . . . . . . . . . . . . . . . 25

Ф. Random Signs . . . . . . . . . . . . . . . . . . . . . . . . . . . . . . . 28

Ð. Other Realizations of DPRM . . . . . . . . . . . . . . . . . . 32

K. Quantum Interference of Strongly Localized Electrons . . . . . . . . . . . . 34

L. The Locator Expansion and Forward Scattering Paths . . . . . . . . . . . . 37

M. Magnetic Field Response . . . . . . . . . . . . . . . . . . . . . . . . 39

N. Unitary Propagation . . . . . . . . . . . . . . . . . . . . . . . . . . 44

O. Unitary Averages . . . . . . . . . . . . . . . . . . . . . . . . . . . . 47

P. Summing all Paths in High Dimensions . . . . . . . . . . . . . . . . . . 52

Q. The Ising Model on a Square Lattice . . . . . . . . . . . . . . . . . . . 57

R. Singular Behavior . . . . . . . . . . . . . . . . . . . . . . . . . . . 62

S. The Two Dimensional Spin Glass . . . . . . . . . . . . . . . . . . . . . 64

T. Results for the Two Dimensional Spin Glass . . . . . . . . . . . . . . . . 67

References . . . . . . . . . . . . . . . . . . . . . . . . . . . . 70 


\section{A. Introduction}

Many physical problems involve calculating sums over paths. Each path could represent one possible physical realization of an object such as a polymer, in which case the weight of the path is the probability of that configuration. The weights themselves could be imaginary as in the case of Feynman paths describing the amplitude for the propagation of a particle. Path integral calculations are now a standard tool of the theoretical physicist, with many excellent books devoted to the subject [1,2].

What happens to sums over paths in the presence of quenched disorder in the medium? Individual paths are no longer weighted simply by their length, but are influenced by the impurities along their route. The sum may be dominated by "optimal" paths pinned to the impurities; the optimal paths usually forming complex hierarchical structures. Physical examples are provided by the interface of the random bond Ising model in two dimensions, and by magnetic flux lines in superconductors. The actual value of the sum naturally depends on the particular realization of randomness and varies from sample to sample. I shall initially describe the problem in the context of the high temperature expansion for the random bond Ising model. Introducing the sums over paths for such a lattice model avoids the difficulties associated with short distance cutoffs. Furthermore, the Ising model is sufficiently well understood to make the nature of various approximations more evident.

The high temperature correlation functions of the Ising model are dominated by the shortest paths connecting the spins. Such configurations, that exclude loops and overhangs, are referred to as directed paths. They dominate the asymptotic behavior of the sum over distances that are much longer than the correlation length. Most of the lectures are devoted to describing the statistical properties of sums over such directed paths. As in all multiplicative noise processes, the probability distribution for the sum is broad. Hence Monte Carlo simulations may not be an appropriate tool for numerical studies; failing to find typical members of the ensemble. Instead, we shall present a transfer matrix method that allows a numerical evaluation of the sum in polynomial time in the length of the path. The results indeed show that the sum has a broad probability distribution that resembles (but is not quite) log-normal.

To obtain analytical information about this probability distribution we shall introduce the replica method for examining the moments. A brief review explains the relationship between the moments, the characteristic function, and cumulants. It can be shown easily that the one dimensional sum has a log-normal distribution. The moments of the sum 
over directed paths in two dimensions can be obtained by using a simple Bethe Ansatz. The implications and limitations of this approach are discussed. There is little analytical information in three and higher dimensions, but a variety of numerical results are available, mostly by taking advantage of a mapping to growing surfaces.

The spin glass problem describes a mixture of ferromagnetic and antiferromagnetic bonds. The resulting sums in the high temperature expansion involve products over a random mixture of positive and negative factors. The calculation of moments is somewhat different from the case of purely positive random bonds. However, we shall demonstrate that the scaling behavior of the distribution is unchanged. A similar sum involving products of random signs is encountered in calculating the probability of an electron tunneling under a random potential. In the strongly localized limit, it is again sufficient to focus on the interference of the forward-scattering (directed) paths. A magnetic field introduces random phases in the sum; while to describe the tunneling of an electron in the presence of spin-orbit scattering requires examining the evolution of a two component spinor and keeping track of products of random matrices. We shall argue that all these cases are in fact described by the same universal probability distribution which, however, does retain some remnant of the underlying symmetries of the original electronic Hamiltonian.

Yet another class of directed paths has been introduced in the context of light scattering in turbulent media. Assuming that inelastic scattering can be neglected, the intensity of the beam is left unchanged, and the evolution is unitary. Due to the constraint of unitarity the resulting directed paths are described by a probability distribution belonging to a new universality class. We shall introduce a discrete matrix model that explicitly takes care of the unitarity constraint. In this model, several properties of the resulting sum over paths can be calculated exactly.

In the last sections of the course we shall go beyond the limitations of directed paths. In a uniform system the sum over all paths is calculated approximately, reproducing meanfield critical behavior. In two dimensions, the sum can be performed exactly for the Ising model. This leads to exact solutions for the pure Ising model, or other uniformly frustrated two dimensional lattices. We shall use this method of exact summation to develop an integer algorithm for obtaining exact partition functions for two dimensional random lattices in polynomial time. Some results are described for the two dimensional spin glass, and contrasted with those obtained from Monte Carlo or transfer matrix methods.

The results described in these notes were the outcome of many collaborations. In particular, I would like to express my thanks to E. Medina, L. Saul, Y. Shapir, and Y.-C. 
Zhang. I am grateful to F. David, P. Ginsparg, and J. Zinn-Justin for organizing the Les Houches summer school, and to the many students who helped me with correcting the lecture notes. The work at MIT was supported by the NSF through grants DMR-93-03667 and PYI/DMR-89-58061.

\section{B. High Temperature Expansions for the Ising Model}

Consider a $d$ dimensional hypercubic lattice of $N$ sites. On each site there is an Ising variable $\sigma_{i}= \pm 1$, and the spins interact through a Hamiltonian

$$
\mathcal{H}=-\sum_{<i j>} J_{i j} \sigma_{i} \sigma_{j}
$$

The symbol $\langle i j\rangle$ implies that the sum is restricted to the $d N$ nearest neighbor bonds on the lattice. The bonds $\left\{J_{i j}\right\}$ are quenched random variables, independently chosen from a probability distribution $p(J)$. For each realization of random bonds, the partition function is computed as

$$
Z\left[J_{i j}\right]=\sum_{\left\{\sigma_{i}\right\}} \exp (-\beta \mathcal{H})=\sum_{\left\{\sigma_{i}\right\}} \prod_{<i j>} e^{K_{i j} \sigma_{i} \sigma_{j}}
$$

where the sums are over the $2^{N}$ possible configurations of spins, $\beta=1 /\left(k_{B} T\right)$ and $K_{i j}=$ $\beta J_{i j}$. To obtain a high temperature expansion, it is more convenient to organize the partition function in powers of $\tanh K_{i j}$. Since $\left(\sigma_{i} \sigma_{j}\right)^{2}=1$, the Boltzmann factor for each bond can be written as

$$
e^{K_{i j} \sigma_{i} \sigma_{j}}=\frac{e^{K_{i j}}+e^{-K_{i j}}}{2}+\frac{e^{K_{i j}}-e^{-K_{i j}}}{2} \sigma_{i} \sigma_{j}=\cosh K_{i j}\left(1+\tau_{i j} \sigma_{i} \sigma_{j}\right),
$$

where $\tau_{i j} \equiv \tanh K_{i j}$ is a good high temperature expansion parameter. Applying this transformation to each bond of the lattice results in

$$
Z\left[J_{i j}\right]=\sum_{\left\{\sigma_{i}\right\}} e^{\sum_{\langle i j\rangle} K_{i j} \sigma_{i} \sigma_{j}}=\bar{C}^{d N} \sum_{\left\{\sigma_{i}\right\}} \prod_{\langle i j\rangle}\left(1+\tau_{i j} \sigma_{i} \sigma_{j}\right)
$$

where

$$
\bar{C}^{d N} \equiv\left(\prod_{\langle i j\rangle} \cosh K_{i j}\right) \quad
$$

The term $\bar{C}^{d N}$ is non-singular, and will be mostly ignored henceforth. The final product in eq. (四) generates $2^{d N}$ terms which can be represented diagrammatically by 
drawing a line connecting sites $i$ and $j$ for each factor of $\tau_{i j} \sigma_{i} \sigma_{j}$. Each site now obtains a factor of $\sigma_{i}^{p_{i}}$, where $0 \leq p_{i} \leq 2 d$ is the number of bonds emanating from $i$. Summing over the two possible values $\sigma_{i}= \pm 1$, gives a factor of 2 if $p_{i}$ is even and 0 is $p_{i}$ is odd. Thus the only graphs that survive the sum have an even number of lines passing through each site. The resulting graphs are collections of closed paths $\mathcal{G}$ on the lattice. The contribution of each graph is the product of $\tau_{i j}$ for the bonds making up the graph, resulting in

$$
Z\left[J_{i j}\right]=2^{N} \times \bar{C}^{d N} \sum_{\mathcal{G}}\left(\prod_{\langle i j\rangle \in \mathcal{G}} \tau_{i j}\right)
$$

For a $d$-dimensional hypercubic lattice the smallest closed graph is a square of 4 bonds and the next graph has 6 bonds. Thus,

$$
Z\left[J_{i j}\right]=2^{N} \times \bar{C}^{d N}\left[1+\sum_{P} \tau_{P 1} \tau_{P 2} \tau_{P 3} \tau_{P 4}+\mathcal{O}\left(\tau^{6}\right)+\cdots\right],
$$

where the sum runs over the $N d(d-1) / 2$ plaquettes on the lattice and $\tau_{P \alpha}$ indicate the four bonds along each plaquette. A quench averaged free energy is now obtained as

$$
\overline{\frac{\ln Z}{N}}=\ln 2+d \overline{\ln \cosh K}+\frac{d(d-1)}{2} \bar{\tau}^{4}+\cdots,
$$

where the over-lines indicate averages over the probability distribution $p(J)$.

The same method can be used to obtain expansions for various spin operators. For example the two spin correlation function is given by

$$
\left\langle\sigma_{m} \sigma_{n}\right\rangle=\sum_{\left\{\sigma_{i}\right\}} \frac{e^{\sum_{\langle i j\rangle} K_{i j} \sigma_{i} \sigma_{j}}}{Z} \sigma_{m} \sigma_{n}=\frac{\bar{C}^{d N}}{Z} \sum_{\left\{\sigma_{i}\right\}} \sigma_{m} \sigma_{n} \prod_{\langle i j\rangle}\left(1+\tau_{i j} \sigma_{i} \sigma_{j}\right)
$$

The terms in the numerator involve an additional factor of $\sigma_{m} \sigma_{n}$. To get a finite value after summing over $\sigma_{m}= \pm 1$ and $\sigma_{n}= \pm 1$ we have to examine graphs with an odd number of bonds emanating from these external sites. After cancelling the common factors between the numerator and denominator, we obtain

$$
\left\langle\sigma_{m} \sigma_{n}\right\rangle=\frac{\sum_{\mathcal{G}_{m n}}\left(\prod_{\langle i j\rangle \in \mathcal{G}_{m n}} \tau_{i j}\right)}{\sum_{\mathcal{G}}\left(\prod_{\langle i j\rangle \in \mathcal{G}} \tau_{i j}\right)} .
$$

Whereas the graphs in $\mathcal{G}$ have an even number of bonds going through each site, those of $\mathcal{G}_{m n}$ have an odd number of bonds going through the external points $m$ and $n$. This procedure can be generalized to multiple spin correlation functions. 


\section{Characteristic Functions and Cumulants}

As equations (6) and (8) indicate, the partition function and correlation functions of the random system are themselves random quantities, dependent on all the bonds $K_{i j}$. It may not be sufficient to just characterize the mean value of $Z$ (or $\ln Z$ ), since the full information about these fluctuating quantities is only contained in their respective probability distributions $p(Z)$ and $p\left(\left\langle\sigma_{m} \sigma_{n}\right\rangle\right)$. It is thus important to learn to characterize and manipulate probability distributions, necessitating the short digression taken in this section to define various notations I shall use in describing such random quantities.

Consider a continuous random variable $x$, whose outcome is a real number $E$.

- The cumulative probability function $(\mathrm{CPF}) P(x)$, is the probability of an outcome with any value less than $x$, i.e. $P(x)=$ prob. $(E \leq x)$. $P(x)$ must be a monotonically increasing function of $x$, with $P(-\infty)=0$ and $P(+\infty)=1$.

- The probability density function (PDF), is defined by $p(x) \equiv d P(x) / d x$. Hence, $p(x) d x=$ prob. $(x<E<x+d x)$. As a probability density, it is positive, and normalized such that

$$
P(\infty)=\int_{-\infty}^{\infty} d x p(x)=1
$$

- The expectation value of any function $F(x)$, of the random variable is

$$
\langle F(x)\rangle=\int_{-\infty}^{\infty} d x p(x) F(x)
$$

The function $F(x)$ is itself a random variable, with an associated PDF of $p_{F}(f) d f=$ prob. $(f<F(x)<f+d f)$. There may be multiple solutions $x_{i}$, to the equation $F(x)=f$, and

$$
p_{F}(f) d f=\sum_{i} p\left(x_{i}\right) d x_{i} \quad \Rightarrow \quad p_{F}(f)=\sum_{i} p\left(x_{i}\right)\left|\frac{d x}{d F}\right|_{x=x_{i}} .
$$

The factors of $|d x / d F|$ are the Jacobians associated with the change of variables from $x$ to $F$.

- Moments of the PDF are expectation values for powers of the random variable. The $n^{\text {th }}$ moment is

$$
\left\langle x^{n}\right\rangle=\int d x p(x) x^{n} .
$$

- The characteristic function, is the generator of moments of the distribution. It is simply the Fourier transform of the PDF, defined by

$$
\tilde{p}(k)=\left\langle e^{-i k x}\right\rangle=\int d x p(x) e^{-i k x} .
$$


Moments of the distribution can be obtained by expanding $\tilde{p}(k)$ in powers of $k$,

$$
\tilde{p}(k)=\left\langle\sum_{n=0}^{\infty} \frac{(-i k)^{n}}{n !} x^{n}\right\rangle=\sum_{n=0}^{\infty} \frac{(-i k)^{n}}{n !}\left\langle x^{n}\right\rangle
$$

- The cumulant generating function is the logarithm of the characteristic function. Its expansion generates the cumulants of the distribution defined through

$$
\ln \tilde{p}(k)=\sum_{n=1}^{\infty} \frac{(-i k)^{n}}{n !}\left\langle x^{n}\right\rangle_{c}
$$

Relations between moments and cumulants can be obtained by expanding the logarithm of $\tilde{p}(k)$ in eq. (15), and using

$$
\ln (1+\epsilon)=\sum_{n=1}^{\infty}(-1)^{n+1} \frac{\epsilon^{n}}{n}
$$

The first four cumulants are called the mean, variance, skewness, and curtosis of the distribution respectively, and are obtained from the moments as

$$
\begin{aligned}
\langle x\rangle_{c} & =\langle x\rangle, \\
\left\langle x^{2}\right\rangle_{c} & =\left\langle x^{2}\right\rangle-\langle x\rangle^{2}, \\
\left\langle x^{3}\right\rangle_{c} & =\left\langle x^{3}\right\rangle-3\left\langle x^{2}\right\rangle\langle x\rangle+2\langle x\rangle^{3}, \\
\left\langle x^{4}\right\rangle_{c} & =\left\langle x^{4}\right\rangle-4\left\langle x^{3}\right\rangle\langle x\rangle-3\left\langle x^{2}\right\rangle^{2}+12\left\langle x^{2}\right\rangle\langle x\rangle^{2}-6\langle x\rangle^{4} .
\end{aligned}
$$

The cumulants are usually the most compact way of describing a PDF. An important theorem allows easy computation of moments in terms of the cumulants: Represent the $n^{\text {th }}$ cumulant graphically as a connected cluster of $n$ points. The $m^{\text {th }}$ moment is then obtained by summing all clusters (connected or disconnected) of $n$ points; the contribution of each cluster being the product of the connected cumulants that it represents. Using this result the first four moments are easily computed as

$$
\begin{aligned}
\langle x\rangle & =\langle x\rangle_{c} \\
\left\langle x^{2}\right\rangle & =\left\langle x^{2}\right\rangle_{c}+\langle x\rangle_{c}^{2} \\
\left\langle x^{3}\right\rangle & =\left\langle x^{3}\right\rangle_{c}+3\left\langle x^{2}\right\rangle_{c}\langle x\rangle_{c}+\langle x\rangle_{c}^{3} \\
\left\langle x^{4}\right\rangle & =\left\langle x^{4}\right\rangle_{c}+4\left\langle x^{3}\right\rangle_{c}\langle x\rangle_{c}+3\left\langle x^{2}\right\rangle_{c}^{2}+6\left\langle x^{2}\right\rangle_{c}\langle x\rangle_{c}^{2}+\langle x\rangle_{c}^{4} .
\end{aligned}
$$


- The normal (Gaussian) distribution describes a continuous real random variable $x$, with

$$
p(x)=\frac{1}{\sqrt{2 \pi \sigma^{2}}} \exp \left[-\frac{(x-\lambda)^{2}}{2 \sigma^{2}}\right] .
$$

The corresponding characteristic function also has a Gaussian form,

$$
\tilde{p}(k)=\int_{-\infty}^{\infty} d x \frac{1}{\sqrt{2 \pi \sigma^{2}}} \exp \left[-\frac{(x-\lambda)^{2}}{2 \sigma^{2}}-i k x\right]=\exp \left[-i k \lambda-\frac{k^{2} \sigma^{2}}{2}\right] .
$$

Cumulants of the distribution can be identified from $\ln \tilde{p}(k)=-i k \lambda-k^{2} \sigma^{2} / 2$, using eq. (16), as

$$
\langle x\rangle_{c}=\lambda \quad, \quad\left\langle x^{2}\right\rangle_{c}=\sigma^{2} \quad, \quad\left\langle x^{3}\right\rangle_{c}=\left\langle x^{4}\right\rangle_{c}=\cdots=0 \quad .
$$

The normal distribution is thus completely specified by its two first cumulants. This makes the computation of moments using the cluster expansion (eqs. (19)) particularly simple, and

$$
\begin{aligned}
\langle x\rangle & =\lambda, \\
\left\langle x^{2}\right\rangle & =\sigma^{2}+\lambda^{2}, \\
\left\langle x^{3}\right\rangle & =3 \sigma^{2} \lambda+\lambda^{3}, \\
\left\langle x^{4}\right\rangle & =3 \sigma^{4}+6 \sigma^{2} \lambda^{2}+\lambda^{4} \quad, \quad \cdots \quad .
\end{aligned}
$$

- The central limit theorem describes the probability distribution for a sum $\mathcal{S}=\sum_{i=1}^{N} x_{i}$ over a large number of random variables. Cumulants of the sum are given by,

$$
\langle\mathcal{S}\rangle_{c}=\sum_{i=1}^{N}\left\langle x_{i}\right\rangle_{c} \quad, \quad\left\langle\mathcal{S}^{2}\right\rangle_{c}=\sum_{i, j}^{N}\left\langle x_{i} x_{j}\right\rangle_{c} \quad, \quad \cdots
$$

If the random variables are independent, $p(\mathbf{x})=\prod p_{i}\left(x_{i}\right)$, and $\tilde{p}_{\mathcal{S}}(k)=\prod \tilde{p}_{i}(k)$. The crosscumulants in eq. (24) vanish, and the $n^{\text {th }}$ cumulant of $\mathcal{S}$ is simply the sum of the individual cumulants, $\left\langle\mathcal{S}^{n}\right\rangle_{c}=\sum_{i=1}^{N}\left\langle x_{i}^{n}\right\rangle_{c}$. When all the $N$ random variables are independently taken from the same distribution $p(x)$, this implies $\left\langle\mathcal{S}^{n}\right\rangle_{c}=N\left\langle x^{n}\right\rangle_{c}$. For large values of $N$, the average value of the sum is proportional to $N$ while fluctuations around the mean, as measured by the standard deviation, grow only as $\sqrt{N}$. The random variable $y=\left(\mathcal{S}-N\langle x\rangle_{c}\right) / \sqrt{N}$, has zero mean, and cumulants that scale as $\left\langle y^{m}\right\rangle_{c} \propto N^{1-m / 2}$. As $N \rightarrow \infty$, only the second cumulant survives and the PDF for $y$ converges to the normal distribution,

$$
\lim _{N \rightarrow \infty} p\left(y=\frac{\sum_{i=1}^{N} x_{i}-N\langle x\rangle_{c}}{\sqrt{N}}\right)=\frac{1}{\sqrt{2 \pi\left\langle x^{2}\right\rangle_{c}}} \exp \left(-\frac{y^{2}}{2\left\langle x^{2}\right\rangle_{c}}\right) .
$$


The convergence of the PDF for the sum of many random variables to a normal distribution is a most important result in the context of statistical mechanics where such sums are frequently encountered. The central limit theorem proves a more general form of this result: It is not necessary for the random variables to be independent, as the condition $\sum_{i_{1}, \cdots, i_{m}}^{N}\left\langle x_{i_{1}} \cdots x_{i_{m}}\right\rangle_{c} \ll \mathcal{O}\left(N^{m / 2}\right)$, is sufficient for the validity of eq. (25).

\section{The One Dimensional Chain}

The graphical method provides a rapid way of solving the Ising model at zero field in $d=1$. We can compare and contrast the solutions on chains with open and closed (periodic) boundary conditions.

1. An open chain of $N$ sites has $N-1$ bonds. It is impossible to draw any closed graphs on such a lattice, and hence

$$
Z=2^{N} \prod_{\alpha=1}^{N-1} \cosh K_{\alpha} \times 1 \Longrightarrow \frac{\overline{\ln Z}}{N}=\overline{\ln [2 \cosh K]}-\frac{\overline{\ln [\cosh K]}}{N}
$$

where $K_{\alpha} \equiv K_{\alpha \alpha+1}$. There is also only one graph that contributes to the two point correlation function,

$$
\left\langle\sigma_{m} \sigma_{n}\right\rangle=\sum_{\left\{\sigma_{i}\right\}} \frac{e^{\sum_{i} K_{i} \sigma_{i} \sigma_{i+1}}}{Z} \sigma_{m} \sigma_{n}=\prod_{\alpha=m}^{n-1} \tau_{\alpha}
$$

2. A closed chain has the same number of sites and bonds, $N$. It is now possible to draw a closed graph that circles the whole chain, and

$$
\begin{aligned}
& Z=2^{N}\left(\prod_{\alpha=1}^{N} \cosh K_{\alpha}\right)\left[1+\left(\prod_{\alpha=1}^{N} \tau_{\alpha}\right)\right] \quad \Longrightarrow \\
& \frac{\overline{\ln Z}}{N} \approx \overline{\ln [2 \cosh K]}+\frac{1}{N} \bar{\tau}^{N}
\end{aligned}
$$

The difference between the quenched free energies of closed and open chains is a surface term of the order of $1 / N$, and an exponential decay reflecting the interaction between edges, both vanishing in the thermodynamic limit of $N \rightarrow \infty$. The correlation function can again be calculated from eq. (8). There are two paths connecting the points $m$ and $n$, along the two possible directions on the chain, giving

$$
\left\langle\sigma_{m} \sigma_{n}\right\rangle=\frac{\left[\prod_{\alpha=m}^{n-1} \tau_{\alpha}+\prod_{\alpha=n}^{m-1} \tau_{\alpha}\right]}{\left[1+\left(\prod_{\alpha=1}^{N} \tau_{\alpha}\right)\right]}
$$


Since the partition function of the open chain is the sum of $N-1$ independent variables,

$$
\frac{\ln Z}{N}=\ln 2+\sum_{\alpha=1}^{N-1} \frac{\ln \cosh K_{\alpha}}{N},
$$

we can use the central limit theorem to conclude that as $N \rightarrow \infty$ the probability distribution $p(\ln Z / N)$, is a gaussian with mean

$$
\overline{\ln Z}=N(\ln 2+\overline{\ln \cosh K})
$$

and variance

$$
\overline{(\ln Z)_{c}^{2}} \equiv \overline{(\ln Z)^{2}}-\overline{(\ln Z)}^{2}=N \overline{(\ln \cosh K)_{c}^{2}}
$$

(Note that I have ignored the small difference between $N$ and $N-1$ in the thermodynamic limit.) Similarly, for the correlation function of two points separated by a distance $t$, we have

$$
\ln \left\langle\sigma_{0} \sigma_{t}\right\rangle=\sum_{\alpha=0}^{t-1} \ln \tau_{\alpha} .
$$

As long as the random variables on the bonds are independently distributed, the cumulants of $\ln \left\langle\sigma_{0} \sigma_{t}\right\rangle$ are given by,

$$
\left\{\begin{array}{rl}
\overline{\ln \left\langle\sigma_{0} \sigma_{t}\right\rangle} & =t \overline{\ln \tanh K} \\
\overline{\left(\ln \left\langle\sigma_{0} \sigma_{t}\right\rangle\right)_{c}^{2}} & =t \overline{(\ln \tanh K)_{c}^{2}} \\
\vdots & \vdots \\
\overline{\left(\ln \left\langle\sigma_{0} \sigma_{t}\right\rangle\right)_{c}^{p}} & =t \overline{(\ln \tanh K)_{c}^{p}}
\end{array} .\right.
$$

In the following sections we shall try to obtain similar information about probability distribution functions for the partition and correlation functions in higher dimensions. To do this we shall employ the replica method for calculating the moments of the distribution. For example, the cumulants of the free energy are given by

$$
\overline{Z^{n}}=\overline{e^{n \ln Z}}=\exp \left[n \overline{\ln Z}+\frac{n^{2}}{2} \overline{(\ln Z)_{c}^{2}}+\cdots+\frac{n^{p}}{p !} \overline{(\ln Z)_{c}^{p}}+\cdots\right],
$$

where we have taken advantage of eq. (16), replacing $(-i k)$ with $n$. Usually, the moments on the left hand side of the above equation are known only for integer $n$, while the evaluation of the cumulants on the right hand side relies on an expansion around $n=0$. This is one of the difficulties associated with the problem of deducing a probability distribution $p(x)$, from the knowledge of its moments $\overline{x^{n}}$. There is in fact a rigorous theorem that 
the probability distribution cannot be uniquely inferred if its $n^{\text {th }}$ moment increases faster than $n$ ! [3]. Most of the distributions of interest to us (such as the above log-normal) do not satisfy this condition! Similar problems are encountered in the replica studies of spin glasses [4]. It turns out that many of the difficulties associated with a rigorous inversion are related to the tail of the distribution. Most of the information of interest to us is contained in the "bulk" of the distribution which is easier to investigate. Rather than taking a rigorous approach to the problem, we shall illustrate the difficulties and their resolution by examining the one dimensional case in detail since it actually presents the worst case scenario for the inverting of moments.

We used the central limit theorem to deduce that the probability distribution for $\left\langle\sigma_{0} \sigma_{t}\right\rangle$ is log-normal. Its moments are computed from,

$$
\overline{\left\langle\sigma_{0} \sigma_{t}\right\rangle^{n}}=\prod_{\alpha=0}^{t-1} \overline{\tau_{\alpha}^{n}}=\left(\overline{e^{n \ln \tau}}\right)^{t}=\exp \left[t \sum_{p} \frac{n^{p}}{p !} \overline{(\ln \tau)_{c}^{p}}\right] .
$$

Let us consider a binary distribution in which $\tau$ takes two positive values of $\tau_{1}$ and $\tau_{2}>\tau_{1}$ with equal probability. Then

$$
\overline{\tau^{n}}=\frac{\tau_{1}^{n}+\tau_{2}^{n}}{2}
$$

and the generating function for the cumulants of the correlation function is

$$
\begin{array}{rl}
\ln \overline{\tau^{n}} & =n \ln \tau_{1}+\ln \left(\frac{1+\left(\tau_{2} / \tau_{1}\right)^{n}}{2}\right) \\
& =n \ln \tau_{1}+\ln \left(\frac{1+1+n \ln \left(\tau_{2} / \tau_{1}\right)+n^{2} / 2 \ln ^{2}\left(\tau_{2} / \tau_{1}\right)+\cdots}{2}\right) \\
n \rightarrow 0 & n \ln \tau_{1}+\ln \left[1+\frac{n}{2} \ln \left(\frac{\tau_{2}}{\tau_{1}}\right)+\frac{n^{2}}{4} \ln ^{2}\left(\frac{\tau_{2}}{\tau_{1}}\right)+\cdots\right] \\
& =n \ln \tau_{1}+\frac{n}{2} \ln \left(\frac{\tau_{2}}{\tau_{1}}\right)+\frac{n^{2}}{8} \ln ^{2}\left(\frac{\tau_{2}}{\tau_{1}}\right)+\cdots \\
& =n \ln \left(\sqrt{\tau_{1} \tau_{2}}\right)+\frac{n^{2}}{8} \ln ^{2}\left(\frac{\tau_{2}}{\tau_{1}}\right)+\cdots
\end{array}
$$

Combining eqs. (36) and (38), the cumulants of the correlation function are given by

$$
\left\{\begin{array}{rl}
\overline{\ln \left\langle\sigma_{0} \sigma_{t}\right\rangle} & =t \ln \left(\sqrt{\tau_{1} \tau_{2}}\right) \\
\overline{\ln \left\langle\sigma_{0} \sigma_{t}\right\rangle_{c}^{2}} & =\frac{t}{4} \ln ^{2}\left(\tau_{2} / \tau_{1}\right) \\
& \vdots
\end{array} .\right.
$$


While it is true that $\ln \left\langle\sigma_{0} \sigma_{t}\right\rangle$ is normally distributed for large $t$, we should be careful about the order of limits in terminating the power series in the exponent at the second order. If we do so, from

$$
\overline{\left\langle\sigma_{0} \sigma_{t}\right\rangle^{n}} \approx \exp \left[t\left(n a_{1}+n^{2} a_{2}\right)\right],
$$

we should not infer anything about the high moments $(n \rightarrow \infty)$ and the tail of the distribution. Otherwise (since $a_{2}>0$ ), we would conclude that sufficiently large moments of $\left\langle\sigma_{0} \sigma_{t}\right\rangle$ diverge with separation; a clearly false conclusion as $\left\langle\sigma_{0} \sigma_{t}\right\rangle$ is bounded by unity! The exact result is that

$$
\lim _{n \rightarrow \infty}\left\langle\sigma_{0} \sigma_{t}\right\rangle^{n}=\frac{\tau_{2}^{n t}}{2^{t}}
$$

i.e., the high moments are almost entirely dominated by the one exceptional sample in which all bonds are equal to $\tau_{2}$. We can summarize the situation as follows: The "bulk" of the probability distribution for $\ln \left\langle\sigma_{0} \sigma_{t}\right\rangle$ is described by the small moments $(n \rightarrow 0)$, while the tail of the distribution is governed by the large moments $(n \rightarrow \infty)$. We should have a clear idea of the crossover point $n^{*}$ in applying the replica method. For the above one dimensional example, an estimate of $n^{*}$ is given by the ratio of the successive terms in the expansion, i.e.

$$
n^{*}=\frac{1}{\ln \left(\tau_{2} / \tau_{1}\right)} \text {. }
$$

Note that as $\tau_{2} / \tau_{1}$ becomes large, $n^{*}$ decreases, possibly becoming smaller than unity. This does not imply that we should conclude that $\ln \left\langle\sigma_{0} \sigma_{t}\right\rangle$ is not normally distributed, just that the tail of the distribution is more prominent. Failure to appreciate this point is the source of some misunderstandings on the use of the replica method [5].

Clearly, it is possible to come up with many different microscopic distributions $p(\tau)$, which result in the same first two cumulants in eqs. (39), but different higher cumulants. All these cases lead to the same universal bulk probability distribution for $\ln \left\langle\sigma_{0} \sigma_{t}\right\rangle$ at large $t$, but very different tails. Thus the non-uniqueness of the overall probability in this example has to do with the rather uninteresting (and nonuniversal) behavior of the tail of the distribution. The correct interpretation of eqs. (39) is that the mean value for the logarithm of the correlation function grows linearly with the separation $t$. In analogy with pure systems, we can regard the coefficient of this decay as the inverse correlation length, i.e. $\xi^{-1}=-\ln \sqrt{\tau_{1} \tau_{2}}$. However, due to randomness in the medium, correlations have different decays between different realizations (and between different points in the same realization). The variations in this "inverse correlation length" are scale dependent and fall off as $1 / \sqrt{t}$. In the next sections we shall attempt to generalize these results to higher dimensions. 


\section{E. Directed Paths and the Transfer Matrix}

Calculation of the correlation function in higher dimensions is complicated by the presence of an exponentially large number of paths connecting any pair of points. On physical grounds we expect the high temperature phase to be disordered, with correlations that decay exponentially as a function of the separation $t$. The essence of this exponential decay is captured by the lowest order terms in the high temperature expansion. The first term in the series comes from the shortest path connecting the two points. Actually, along a generic direction on a hypercubic lattice there are many paths that have the same shortest length. (In two dimensions, the length of the shortest path connecting $(0,0)$ to $(t, x)$ is the "Manhattan" distance $|t|+|x|$.) The number of paths grows from a minimum of 1 along a lattice direction to a maximum of $d$ per step along the diagonal. (The number of paths on the square lattice is $(t+x) ! /(t ! x !)$.) Thus the decay of correlations depends on orientation, a consequence of the anisotropy of the hypercubic lattice. (Note that this anisotropy is absent at distances less than the correlation length. We thus don't have to worry about anisotropy effects in discretizing critical (massless) theories on a lattice.)

In a uniform system these shortest paths are sufficient to capture the essence of correlation functions of the high temperature phase: An exponential decay with separation which is generic to all spin systems. As temperature is reduced, more complicated paths (e.g. with loops and overhangs) start contributing to the sum. Although the contribution of these paths decays exponentially in their length, their number grows exponentially. Ultimately at the critical point this "entropic" increase in the number of paths overcomes the "energetic" decrease due to the factors of $\tau<1$, and paths of all length become important below $T_{c}$. However, throughout the high temperature phase, it is possible to examine the paths at the coarse grained scale where no loops and overhangs are present. The scale of such structures is roughly the correlation length $\xi$, and if we use $\xi$ as the unit of a coarse-grained lattice, the paths contributing to the correlation function are directed.

Let me define "directed paths" more carefully: Between any pair of points on the lattice we can draw an imaginary line which I shall refer to as the "time" axis $t$. Transverse directions (perpendicular to the $t$ axis) are indicated by $\vec{x}$. Directed paths are similar to the worldlines of a particle $\vec{x}(t)$ in time; they exclude any path from the initial to the final point that has steps opposite to the main time direction. The question of the validity of this approximation, and the importance of the neglected loops continually comes up. This is possibly because it is more common to think about the vicinity of the critical 
point where loops of all sizes are present and equally important. Away from the critical point, we have to distinguish between properties at scales smaller and larger than the correlation length $\xi$; there is no other length scale (except for the lattice spacing) in the problem. Limiting the sum for the correlation function to directed paths is only useful for separations $t \gg \xi$. Loops, overhangs, and additional structures occur up to size $\xi$ (the only length scale present) and can be removed by coarse graining such that the lattice spacing is larger than or equal to $\xi$. This is automatically the case in a high temperature expansion since $\xi$ is initially less than a lattice spacing. By the same argument, we may also neglect the closed loops (vacuum bubbles) generated by the denominator of eq. (9).

In this section I shall demonstrate how sums over directed paths in the uniform system can be calculated exactly by transfer matrix methods. The method can also be generalized to random systems, providing an algorithm for summing all paths in polynomial time. For ease of visualization, I shall demonstrate this method in two dimensions; the results are easily generalizable to higher dimensions. Also to emphasize the general features of the transfer matrix method, we shall compare and contrast the behavior of correlations along the axis and the diagonal of the square lattice.

To calculate the correlation function $\left\langle\sigma_{0,0} \sigma_{0, t}\right\rangle$, on a non-random square lattice, we shall focus on directed paths oriented along the main axis of the square. These paths are specified by a set of transverse coordinates $\left(x_{0}, x_{1}, x_{2}, \cdots, x_{t}\right)$, with $x_{0}=x_{t}=0$. Of course, there is only one shortest path with all $x_{i}$ equal to zero, but we would like to explore the corrections due to longer directed paths. Consider the set of quantities

$$
\langle x, t|W| 0,0\rangle=\text { sum over paths from }(0,0) \text { to }(x, t) \equiv W(x, t)
$$

The calculation of $W(x, t)$ is easily accomplished by taking advantage of its Markovian property: Each step of a path proceeds from its last location and is independent of the previous steps. Hence $W$ can be calculated recursively from,

$$
\begin{aligned}
W(x, t+1) & =\tau\left[W(x, t)+\tau(W(x-1, t)+W(x+1, t))+\mathcal{O}\left(\tau^{2}\right)\right] \\
& \equiv \sum_{x^{\prime}}\left\langle x|T| x^{\prime}\right\rangle W\left(x^{\prime}, t\right)
\end{aligned}
$$

where we have introduced a transfer matrix,

$$
\left\langle x|T| x^{\prime}\right\rangle=\tau \delta_{x, x^{\prime}}+\tau^{2}\left(\delta_{x, x^{\prime}+1}+\delta_{x, x^{\prime}-1}\right)+\mathcal{O}\left(\tau^{3}\right) .
$$


If we treat the values of $W$ at a particular $t$ as a vector, eq. (44) can be iterated as,

$$
\underline{W}(t)=T \underline{W}(t-1)=\cdots=T^{t} \underline{W}(0),
$$

starting from

$$
\underline{W}(0)=\left(\begin{array}{c}
\vdots \\
\tau \\
1 \\
\tau \\
\vdots
\end{array}\right)
$$

The calculations are simplified by diagonalizing the matrix $T$, using the Fourier basis $\langle x \mid q\rangle=e^{i q \cdot x} / \sqrt{N}$, as

$$
T(q)=\tau(1+2 \tau \cos q+\cdots)=\tau \exp \left[2 \tau\left(1-\frac{q^{2}}{2}+\cdots\right)\right] .
$$

In this basis, $W$ is calculated as

$$
\begin{aligned}
W(x, t) & =\left\langle x\left|T^{t}\right| 0\right\rangle=\sum_{q}\langle x \mid q\rangle T(q)^{t}\langle q \mid 0\rangle \\
& =\tau^{t} e^{2 \tau t} \quad \int \frac{d q}{2 \pi} \exp \left[i q x-q^{2} \tau t+\cdots\right] \\
& =\exp \left[t\left(\ln \tau+2 \tau+\mathcal{O}\left(\tau^{2}\right)\right)\right] \times \frac{1}{\sqrt{4 \pi \tau t}} \exp \left[-\frac{x^{2}}{4 \tau t}\right]
\end{aligned}
$$

The result is proportional to a gaussian form in $x$ of width $\sqrt{2 \tau t}$. The exponential decay with $\xi^{-1}=\ln (1 / \tau)-2 \tau+\mathcal{O}\left(\tau^{2}\right)$ at $x=0$ is accompanied by a subleading $1 / \sqrt{t}$.

The corresponding calculation of paths along the diagonal, contributing to $\left\langle\sigma_{0,0} \sigma_{0, t}\right\rangle$, is even simpler. (Note that the $t$ and $x$ axes are rotated by $45^{\circ}$ compared to the previous example.) At each step the path may proceed up or down, leading to the recursion relation

$$
W(x, t+1)=\tau(W(x-1, t)+W(x+1, t)) \equiv \sum_{x^{\prime}}\left\langle x|T| x^{\prime}\right\rangle W\left(x^{\prime}, t\right) \quad,
$$

with the transfer matrix

$$
\left\langle x|T| x^{\prime}\right\rangle=\tau\left(\delta_{x, x^{\prime}+1}+\delta_{x, x^{\prime}-1}\right) \quad \Longrightarrow \quad T(q)=2 \tau \cos q
$$

The calculation of $W$ proceeds as before,

$$
\begin{aligned}
W(x, t) & =\left\langle x\left|T^{t}\right| 0\right\rangle=\sum_{q}\langle x \mid q\rangle T(q)^{t}\langle q \mid 0\rangle \\
& =\int \frac{d q}{2 \pi}(2 \tau)^{t}(\cos q)^{t} e^{i q x} \\
& \approx(2 \tau)^{t} \times \frac{1}{\sqrt{2 \pi t}} \exp \left[-\frac{x^{2}}{2 t}\right]
\end{aligned}
$$


where the final result is obtained by a saddle point evaluation of the integral, essentially replacing $\cos ^{t} q$ with $\exp \left(-q^{2} t / 2\right)$.

The similarity between eqs. (49) and (52) is apparent. Note that in both cases the leading exponential decay is determined by $T(q=0)$, i.e.

$$
W(0, t) \approx \lambda_{\max }^{t}=T(q=0)^{t}
$$

This is an example of the dominance of the largest eigenvalue in the product of a large number of matrices. There is a corresponding ground state dominance in the evolution of quantum systems. The similarities become further apparent by taking the continuum limit of the recursion relations, which are obtained by regarding $W(x, t)$ as a smooth function, and expanding in the derivatives. From eq. (44), we obtain

$$
W+\frac{\partial W}{\partial t}+\cdots=\tau W+\tau^{2}\left(2 W+\frac{\partial^{2} W}{\partial x^{2}}+\cdots\right)
$$

while eq. (50) leads to

$$
W+\frac{\partial W}{\partial t}+\cdots=2 \tau W+\tau \frac{\partial^{2} W}{\partial x^{2}}+\cdots
$$

For large $t$, the function $W$ decays slowly for adjacent points in the $x$ direction, and it is justified to only consider the lowest order derivatives with respect to $x$. The decay factor along the $t$ direction is, however, quite big and we shall keep track of all derivatives in this direction, leading to

$$
e^{\partial_{t}} W=\tau \exp \left[2 \tau+\tau \partial_{x}^{2}+\cdots\right] W
$$

and

$$
e^{\partial_{t}} W=2 \tau \exp \left[\frac{1}{2} \partial_{x}^{2}+\cdots\right] W,
$$

respectively. Both equations can be rearranged (and generalized in higher dimensions) into the differential form,

$$
\frac{\partial W}{\partial t}=-\frac{W}{\xi(\theta)}+\nu(\theta) \nabla^{2} W
$$

where $\xi(\theta)$ and $\nu(\theta)$ are the orientation dependent correlation length and dispersion coefficient. Eq. (58) can be regarded as a diffusion equation in the presence of a sink, or an imaginary-time Schrödinger equation. 
It is of course quite easy to integrate this linear equation to reproduce the results in eqs. (49) and (52). However, it is also possible 11 to express the solution in the form of a continuous path integral. The solution is trivial in Fourier space,

$$
\frac{\partial W(q)}{\partial t}=-\left(\xi^{-1}+\nu q^{2}\right) W \quad \Longrightarrow \quad W(q, t+\Delta t)=e^{-\left(\xi^{-1}+\nu q^{2}\right) \Delta t} W(q, t)
$$

while in real space,

$$
\begin{aligned}
W(x, t+\Delta t) & =\int \frac{d q}{2 \pi} e^{i q x} e^{-\left(\xi^{-1}+\nu q^{2}\right) \Delta t} W(q, t) \\
& =\int \frac{d q}{2 \pi} e^{i q x} e^{\left(-\xi^{-1}-\nu q^{2}\right) \Delta t} \int d x_{t} e^{-i q x_{t}} W\left(x_{t}, t\right) \\
& =\int d x_{t} \exp \left[-\frac{\Delta t}{\xi}-\frac{\left(x-x_{t}\right)^{2}}{4 \nu \Delta t}\right] W\left(x_{t}, t\right) \\
& =\int d x_{t} \exp \left[-\frac{\Delta t}{\xi}+\frac{\Delta t}{4 \nu}\left(\frac{x_{t+\Delta t}-x_{t}}{\Delta t}\right)^{2}\right] W\left(x_{t}, t\right)
\end{aligned}
$$

which is just a continuum version of eqs. (44) and (50). We can subdivide the interval $(0, t)$ into $N$ subintervals of length $\Delta t=t / N$. In the limit of $N \rightarrow \infty$, recursion of eq. (60) gives

$$
W(x, t)=\int_{(0.0)}^{(x, t)} \mathcal{D} x\left(t^{\prime}\right) \exp \left[\int_{0}^{t} d t^{\prime}\left(-\frac{1}{\xi(\theta)}-\frac{\dot{x}^{2}}{4 \nu(\theta)}\right)\right],
$$

where $\dot{x}=d x / d t^{\prime}$, and the integration is over all functions $x\left(t^{\prime}\right)$.

It is instructive to compare the above path integral with the partition function of a string stretched between $(0,0)$ and $(x, t)$,

$$
\begin{aligned}
Z(x, t) & =\int_{(0,0)}^{(x, t)} \mathcal{D} x\left(t^{\prime}\right) \exp \left[-\beta \sigma \int_{0}^{t} d t^{\prime} \sqrt{1+\dot{x}^{2}}\right] \\
& =\int_{(0,0)}^{(x, t)} \mathcal{D} x\left(t^{\prime}\right) \exp \left[-\int_{0}^{t} d t^{\prime}\left(\beta \sigma+\frac{\beta \sigma}{2} \dot{x}^{2}+\cdots\right)\right]
\end{aligned}
$$

where $\sigma$ is the line tension. Whereas for the string $\xi^{-1}=(2 \nu)^{-1}=\beta \sigma$, in general due to the anisotropy of the lattice these quantities need not be equal. By matching solutions at nearby angles of $\theta$ and $\theta+d \theta$, it is possible to obtain a relation between $\xi^{-1}(\theta)$ and $\nu^{-1}(\theta)$. (For a similar relation in the context of interfaces of Ising models, see ref. [6].) However, $\xi(\theta)^{-1}$ calculated from the shortest paths only is singular along the axis $\theta=0$. This is why to calculate the parameter $\nu$ along this direction it is necessary to include longer directed paths. 


\section{F. Moments of the Correlation Function}

We now return to the correlation functions in the presence of random bonds. In the high temperature limit, we can still set

$$
W(x, t) \equiv\left\langle\sigma_{0,0} \sigma_{x, t}\right\rangle=\sum_{P} \prod_{i=1}^{t} \tau_{P i},
$$

where the sum is over all the diagonally oriented directed paths $P$ from $(0,0)$ to $(x, t)$, and the $\tau_{P i}$ denote factors of $\tanh K$ encountered for the random bonds along each path. The $\tau_{i}$ are random variables, independently chosen for each bond. We shall assume that the probability distribution $p(\tau)$ is narrowly distributed around a mean value $\bar{\tau}$ with width $\sigma$. Clearly, $W(x, t)$ is itself a random variable and we would like to find its probability distribution. Rather than directly calculating $p(W)$, we shall first examine its moments $\overline{W^{n}}$.

Calculation of the first moment is trivial: Each factor of $\tau_{i}$ occurs at most once in eq. (63), and hence after averaging,

$$
\overline{W(x, t)} \equiv\left\langle\sigma_{0,0} \sigma_{x, t}\right\rangle=\sum_{P} \bar{\tau}^{t} .
$$

This is precisely the sum encountered in a non-random system, with $\bar{\tau}$ replacing $\tau$. For example, along the square diagonal,

$$
\overline{W(x, t)} \approx(2 \bar{\tau})^{t} \times \frac{1}{\sqrt{2 \pi t}} \exp \left[-\frac{x^{2}}{2 t}\right],
$$

and in general, in the continuum limit,

$$
\frac{\partial \bar{W}}{\partial t}=-\frac{\bar{W}}{\xi}+\nu \frac{\partial^{2} \bar{W}}{\partial x^{2}} .
$$

For the calculation of the second moment we need to evaluate

$$
\overline{W W}=\sum_{P, P^{\prime}} \prod_{i=1}^{t} \overline{\tau_{P i} \tau_{P^{\prime} i}} .
$$

For a particular $i$, there are two possible averages depending on whether or not the two paths cross the same bond,

$$
\overline{\tau_{P i} \tau_{P^{\prime} i}}=\left\{\begin{array}{ll}
\bar{\tau}^{2} & \text { if } P i \neq P^{\prime} i \\
\overline{\tau^{2}} & \text { if } P i=P^{\prime} i
\end{array} .\right.
$$


Since $\overline{\tau^{2}}>\bar{\tau}^{2}$ there is an additional weight for paths that intersect compared to those that don't. This can be regarded as an attraction between the two paths, represented by a Boltzmann weight,

$$
U=\frac{\overline{\tau^{2}}}{\bar{\tau}^{2}}=\frac{\bar{\tau}^{2}+\sigma^{2}}{\bar{\tau}^{2}}=1+\frac{\sigma^{2}}{\bar{\tau}^{2}} \approx e^{\sigma^{2} / \bar{\tau}^{2}}
$$

Including the attraction, the recursion relation for $\overline{W W}$ is,

$$
W_{2}\left(x_{1}, x_{2}, t\right) \equiv \overline{W\left(x_{1}, t\right) W\left(x_{2}, t\right)}=\sum_{x_{1}^{\prime} x_{2}^{\prime}}\left\langle x_{1} x_{2}\left|T_{2}\right| x_{1}^{\prime} x_{2}^{\prime}\right\rangle W\left(x_{1}^{\prime}, x_{2}^{\prime}, t-1\right),
$$

with the two body transfer matrix

$$
\left\langle x_{1} x_{2}\left|T_{2}\right| x_{1}^{\prime} x_{2}^{\prime}\right\rangle=\bar{\tau}^{2}\left(\delta_{x_{1}, x_{1}^{\prime}+1}+\delta_{x_{1}, x_{1}^{\prime}-1}\right)\left(\delta_{x_{2}, x_{2}^{\prime}+1}+\delta_{x_{2}, x_{2}^{\prime}-1}\right)\left(1+(U-1) \delta_{x_{1}, x_{2}} \delta_{x_{1}^{\prime}, x_{2}^{\prime}}\right) .
$$

The significance of the attraction in eq. (69) is as follows: In the random system the paths prefer to pass through regions with particularly favorable values of $\tau$. After performing the quench averaging the paths go through a uniform medium. The tendency for the original paths to bunch up through favorable spots is instead mimicked by the uniform attraction which tends to bundle together the paths representing the higher moments.

In the continuum limit, eq. (70) goes over to a differential equation of the form,

$$
\frac{\partial W_{2}\left(x_{1}, x_{2}, t\right)}{\partial t}=-\frac{2 W_{2}}{\xi}+\nu \frac{\partial^{2} W_{2}}{\partial x_{1}^{2}}+\nu \frac{\partial^{2} W_{2}}{\partial x_{2}^{2}}+u \delta\left(x_{1}-x_{2}\right) W_{2} \equiv-H_{2} W_{2}
$$

with $u \approx \sigma^{2} / \bar{\tau}^{2}$. Alternatively, we could have obtained eq. (72) from the continuum version of the path integral,

$$
\begin{array}{r}
W_{2}\left(x_{1}, x_{2}, t\right)=\int_{(0.0)}^{\left(x_{1}, t\right)} \mathcal{D} x_{1}\left(t^{\prime}\right) \int_{(0.0)}^{\left(x_{2}, t\right)} \mathcal{D} x_{2}\left(t^{\prime}\right) \exp \left[\int_{0}^{t} d t^{\prime} u \delta\left(x_{1}\left(t^{\prime}\right)-x_{2}\left(t^{\prime}\right)\right)\right] \\
\exp \left[\int_{0}^{t} d t^{\prime}\left(-\frac{1}{\xi}-\frac{\dot{x}_{1}^{2}}{4 \nu}\right)\right] \exp \left[\int_{0}^{t} d t^{\prime}\left(-\frac{1}{\xi}-\frac{\dot{x}_{2}^{2}}{4 \nu}\right)\right]
\end{array}
$$

Formally integrating eq. (72) yields $W_{2} \propto \exp \left(-t H_{2}\right)$, which can be evaluated in the basis of eigenvalues of $\mathrm{H}_{2}$ as

$$
W_{2}\left(x_{1}, x_{2}, t\right)=\left\langle x_{1} x_{2}\left|T_{2}^{t}\right| 00\right\rangle=\sum_{m}\left\langle x_{1} x_{2} \mid m\right\rangle e^{-\epsilon_{m} t}\langle m \mid 00\rangle \underset{t \rightarrow \infty}{\underset{\sim}{\approx}} e^{-\varepsilon_{0} t},
$$

where $\left\{\varepsilon_{m}\right\}$ are the eigenenergies of $H_{2}$, regarded as a quantum Hamiltonian. The exponential growth of $W_{2}$ for $t \rightarrow \infty$ is dominated by the ground state $\varepsilon_{0}$. 
The two body Hamiltonian depends only on the relative separation of the two particles. After transforming to the center of mass coordinates,

$$
\left\{\begin{array}{l}
r=x_{1}-x_{2} \\
R=\left(x_{1}+x_{2}\right) / 2
\end{array} \quad \Longrightarrow \quad \partial_{1}^{2}+\partial_{2}^{2}=\frac{1}{2} \partial_{R}^{2}+2 \partial_{r}^{2},\right.
$$

the Hamiltonian reads,

$$
H_{2}=\frac{2}{\xi}-\frac{\nu}{2} \partial_{R}^{2}-2 \nu \partial_{r}^{2}-u \delta(r)
$$

The relative coordinate describes a particle in a delta-function potential, which has a ground state wavefunction

$$
\psi_{0}(r, R) \propto e^{-\kappa|r|}
$$

The value of $\kappa$ is obtained by integrating $H_{2} \psi_{0}$ from $-\epsilon$ to $\epsilon$, and requiring the discontinuity in the logarithmic derivative of $\psi_{0}$ to match the strength of the potential; hence

$$
-2 \nu(-\kappa-\kappa)=u \quad \Longrightarrow \quad \kappa=\frac{u}{4 \nu} \approx \frac{\sigma^{2}}{2 \bar{\tau}^{2}}
$$

The ground state energy of this two particle system is

$$
\varepsilon_{0}=+\frac{2}{\xi}-2 \nu \kappa^{2} \approx \frac{2}{\xi}-\frac{u^{2}}{8 \nu}
$$

The inequality,

$$
\overline{W^{2}(t)}=\exp \left[-\frac{2 t}{\xi}+\frac{u^{2} t}{8 \nu}\right]=\overline{W(t)}^{2} \exp \left(\frac{u^{2} t}{8 \nu}\right) \gg \overline{W(t)}^{2},
$$

implies that the probability distribution for $W(t)$ is quite broad, and becomes progressively wider distributed as $t \rightarrow \infty$.

Higher moments of the sum are obtained from

$$
\overline{W^{n}}=\sum_{P_{1}, \cdots, P_{n}} \prod_{i=1}^{t} \overline{\tau_{P_{1} i} \cdots \tau_{P_{n}} i}
$$

At a particular "time" slice there may or may not be intersections amongst the paths. Let us assume that $\tau$ is Gaussian distributed with a mean $\bar{\tau}$, and a narrow width $\sigma$; then,

$$
\begin{aligned}
\overline{\tau^{m}} & \approx \int \frac{d x x^{m}}{\sqrt{2 \pi \sigma^{2}}} \exp \left[-\frac{(x-\bar{\tau})^{2}}{2 \sigma^{2}}\right] \quad(\text { set } x=\bar{\tau}+\epsilon \text { and expand in } \epsilon) \\
& \approx \int \frac{d \epsilon}{\sqrt{2 \pi \sigma^{2}}}\left(\bar{\tau}^{m}+m \bar{\tau}^{m-1} \epsilon+\frac{m(m-1)}{2} \bar{\tau}^{m-2} \epsilon^{2}+\cdots\right) \exp \left[-\frac{\epsilon^{2}}{2 \sigma^{2}}\right] \\
& \approx \bar{\tau}^{m}+\frac{m(m-1)}{2} \bar{\tau}^{m-2} \sigma^{2}+\cdots \approx \bar{\tau}^{m}\left(1+\frac{m(m-1)}{2} \frac{\sigma^{2}}{\bar{\tau}^{2}}+\cdots\right) \\
& \approx \bar{\tau}^{m} \exp \left[\frac{m(m-1)}{2} u\right]
\end{aligned}
$$


Since there are $m(m-1) / 2$ possibilities for pairing $m$ particles, the above result represents the Boltzmann factor for a pairwise attraction of $u$ for particles in contact. Since $\tau$ is bounded by unity, the approximations leading to eq. (82) must break down for sufficiently large $m$. This implies the presence of three and higher body interactions. Such interactions are usually of higher order and can be safely ignored. For a discussion of these higher order interactions in a similar context see ref. [7].

The continuum version of the resulting path integral is

$$
\begin{aligned}
& W_{n}\left(x_{1}, \cdots, x_{n}, t\right) \equiv \overline{W\left(x_{1}, t\right) \cdots W\left(x_{n}, t\right)}=\int_{(0,0, \cdots, 0)}^{\left(x_{1}, \cdots, x_{n}, t\right)} \mathcal{D} x_{1}\left(t^{\prime}\right) \cdots \mathcal{D} x_{n}\left(t^{\prime}\right) \\
& \exp \left[-\frac{n t}{\xi}-\int_{0}^{t} d t^{\prime}\left(\sum_{\alpha} \frac{\dot{x}_{\alpha}^{2}}{4 \nu}-\frac{u}{2} \sum_{\alpha \neq \beta} \delta\left(x_{\alpha}\left(t^{\prime}\right)-x_{\beta}\left(t^{\prime}\right)\right)\right)\right]
\end{aligned}
$$

and evolves according to

$$
\frac{\partial W_{n}}{\partial t}=-\frac{n W_{n}}{\xi}+\nu \sum_{\alpha=1}^{n} \frac{\partial^{2} W_{n}}{\partial x_{\alpha}^{2}}+\frac{u}{2} \sum_{\alpha \neq \beta} \delta\left(x_{\alpha}(\tau)-x_{\beta}(\tau)\right) W_{n} \equiv-H_{n} W_{n} .
$$

The asymptotic behavior of $W_{n}$ at large $t$ is controlled by the ground state of $H_{n}$. The corresponding wavefunction is obtained by a simple Bethe Ansatz [8], which generalizes eq. (77) to

$$
\psi_{0}\left(x_{1}, \cdots, x_{n}\right) \propto \exp \left[-\frac{\kappa}{2} \sum_{\alpha \neq \beta}\left|x_{\alpha}-x_{\beta}\right|\right] \quad \text { with } \quad \kappa=\frac{u}{4 \nu} .
$$

For each ordering of particles on the line the wave function can be expanded as $\psi_{0} \propto \exp \left[\kappa_{\alpha} x_{\alpha}\right]$, with the "momenta" $\kappa_{\alpha}$ getting permuted for different orderings. For example, if $x_{1}<x_{2}<\cdots<x_{n}$, the momenta are

$$
\kappa_{\alpha}=\kappa[2 \alpha-(n+1)],
$$

forming a so called $n$-string. The kinetic energy is proportional to

$$
\begin{aligned}
S & =\sum_{\alpha=1}^{n}[2 \alpha-(n+1)]^{2}=\sum_{\alpha=1}^{n}\left[(n+1)^{2}-4 \alpha(n+1)+4 \alpha^{2}\right] \\
& =n(n+1)^{2}-4(n+1) \cdot \frac{n(n+1)}{2}+\frac{4 n(n+1)(2 n+1)}{6} \\
& =n(n+1)\left[-(n+1)+\frac{2(2 n+1)}{3}\right]=\frac{n(n+1)(n-1)}{3}
\end{aligned}
$$


leading to the ground state energy

$$
\varepsilon_{0}=\frac{n}{\xi}-\nu \sum_{\alpha=1}^{n} \kappa_{\alpha}^{2}=\frac{n}{\xi}-\frac{\nu \kappa^{2}}{3} n\left(n^{2}-1\right)
$$

Thus the asymptotic behavior of moments of the sum has the form

$$
\lim _{t \rightarrow \infty} \overline{W^{n}(t)}=\exp \left[-\frac{n t}{\xi}+\frac{n\left(n^{2}-1\right) \nu \kappa^{2} t}{3}\right]=\overline{W(t)}^{n} \exp \left(\frac{n\left(n^{2}-1\right) u^{2} t}{48 \nu}\right)
$$

\section{G. The Probability Distribution in Two Dimensions}

It is tempting to use eq. (89) in conjunction with

$$
\lim _{n \rightarrow 0} \ln \left(\overline{W^{n}(t)}\right)=n \overline{\ln W}+\frac{n^{2}}{2} \overline{(\ln W)_{c}^{2}}+\cdots+\frac{n^{p}}{p !} \overline{(\ln W)_{c}^{p}}+\cdots,
$$

to read off the cumulants for the probability distribution for $\ln W$. The key point is the absence of the $n^{2}$ term and the presence of the $n^{3} t$ factor in the exponent of eq. (89), suggesting a third cumulant, and hence fluctuations in $\ln W$ that grow as $t^{1 / 3}$ [9]. However, as discussed before, there are subtleties in trying to deduce a probability distribution from the knowledge of its moments which we need to consider first. Since $W(t)$ is bounded by unity, eq. (89) cannot be valid for arbitrarily large $n$. Our first task is to identify the crossover point $n^{*}$ beyond which this result is no longer correct.

Eq. (88) is obtained for the ground state of $n$ particles subject to a two body interaction in the continuum limit. A simple argument can be used to understand the origin of the $n^{3}$ term in the energy, as well as the limitations of the continuum approach. Let us assume that the $n$ particles form a bound state of size $R$. For large $n$, the energy of such a state can be estimated as

$$
\varepsilon \approx \frac{n}{\xi}+\frac{\nu n}{R^{2}}-\frac{u n^{2}}{R}
$$

A variational estimate is obtained by minimizing the above expression with respect to $R$, resulting in $R \propto \nu /(u n)$ and $\varepsilon \propto u^{2} n^{3} / \nu$. The size of the bound state decreases with increasing $n$, and the continuum approximation breaks down when it becomes of the order of the lattice spacing for $n^{*} \propto \nu / u \approx \bar{\tau}^{2} / \sigma^{2}$. For $n \gg n^{*}$ all the paths collapse together and

$$
\lim _{n \rightarrow \infty} \overline{W^{n}(t)} \simeq\left(2 \overline{\tau^{n}}\right)^{t}
$$


This asymptotic behavior is non-universal and depends on the extreme values of the local probability distribution for $\tau$. Depending on the choice of parameters, $n^{*}$ can be large or small. However, as discussed in the context of the one dimensional problem, its value controls only the relative importance of the tail and the bulk of the probability distribution for $\ln W$. The behavior of the bulk of the distribution is expected to be universal. The crossover at $n^{*}$ is explicitly demonstrated in a simpler model in ref. [10].

Another important consideration is the order of limits. Eq. (89) is obtained by taking the $t \rightarrow \infty$ limit at fixed $n$, while the cumulant series in eq. (90) relies on an expansion around $n \rightarrow 0$ for fixed $t$. The two limits do not commute. In fact, we would naively deduce from eq. (89) that only the third cumulant of $\ln W$ is non-zero. This is incorrect as it is impossible to have a probability distribution with only a third cumulant [11]. The correct procedure [10] is to assume that the singular behavior associated with $n \rightarrow 0$ and $t \rightarrow \infty$ is described by a scaling function of the form $g_{s}\left(n t^{\omega}\right)$. (This is similar to the singularity of the free energy at a critical point with $n^{-1 / \omega}$ playing the role of a correlation length.) If $t \rightarrow \infty$ at fixed $n$, extensivity of the free energy of the $n$ particle system forces $\ln \overline{W^{n}(t)}$ to be proportional to $t$. At the other limit of $n \rightarrow 0$ at fixed $t$, the result is a power series in $n$, i.e.

$$
\ln \overline{W^{n}(t)}=a n t+g_{s}\left(n t^{\omega}\right)= \begin{cases}a n t+\rho n^{1 / \omega} t & \text { for } t \rightarrow \infty \text { at fixed } n \\ a n t+g_{1} n t^{\omega}+g_{2}\left(n t^{\omega}\right)^{2}+\cdots & \text { for fixed } t \text { as } n \rightarrow 0\end{cases}
$$

Note that I have included a non-singular term, ant. Similar considerations have been put forward in ref. [12]. Comparison with eq. (89) gives $\omega=1 / 3$, and we can read off the cumulants of $\ln W$ as

$$
\left\{\begin{aligned}
\overline{\ln W(t)} & =a t+g_{1} t^{1 / 3} \\
\overline{\ln W^{2}(t)_{c}} & =2 g_{2} t^{2 / 3} \\
& \vdots \\
\overline{\ln W^{p}(t)_{c}} & =p ! g_{p} t^{p / 3}
\end{aligned}\right.
$$

The existence of $t^{1 / 3}$ corrections to the quench averaged value of $\ln W(t)$ was first suggested by Bouchaud and Orland [13] and has been numerically verified [14]. The $t^{2 / 3}$ growth of the variance of the probability distribution was obtained by Huse and Henley [15] in the context of interfaces of Ising models at zero temperature where an optimal path dominates the sum. The results remain valid at finite temperatures [16]. Simulations are 
performed by implementing the transfer matrix method numerically. For example, along the diagonal of the square lattice, the recursion relation

$$
W(x, t+1)=\tau_{x, t,-} W(x-1, t)+\tau_{x, t,+} W(x+1, t)
$$

is iterated starting from $W(x, 0)=\delta_{x, 0}$. The random numbers $\tau_{x, t, \sigma}$ are generated as the iteration proceeds. The memory requirement (the arrays $W(x)$ ) depend on the final length $t$; each update requires $t$ operations, and the total execution time grows as $t^{2}$. Thus for a given realization of randomness, exact results are obtained in polynomial time. Of course the results have to be averaged over many realizations of randomness. The typical values of $t$ used in the transfer matrix simulations range from $10^{3}$ to $10^{4}$, with $10^{2}$ to $10^{3}$ realizations. Calculating higher cumulants becomes progressively more difficult. The existence of the third cumulant was verified by Halpin-Healy [17]. A fourth cumulant, growing as $t^{4 / 3}$ was observed by Kim et al. [18]. Starting from the replica result, Zhang [19] proposed an analytical form, $p(\ln W, t) \sim \exp \left(-a|\ln W-\overline{\ln W}|^{3 / 2} / t^{1 / 2}\right)$. While this form captures the correct scaling of free energy fluctuations, it is symmetric about the average value precluding the observed finite third cumulant. This deficiency was remedied by Crisanti et al. 20] who generalized the above probability to one with different coefficients $a_{ \pm}$on the two sides of the mean value.

So far, we focused on the asymptotic behavior of $W(x, t)$ at large $t$, ignoring the dependence on the transverse coordinate. For the pure problem, the dependence of $W$ on the transverse coordinate is a Gaussian, centered at the origin, with a width that grows as $t^{1 / 2}$. The full dependence is obtained in the pure problem by including the band of eigenvalues with energies close to the ground state. Unfortunately, determining the appropriate eigenvalues for the interacting problem is rather difficult. In addition to the eigenvalues obtained by simply multiplying eq. (85) by $\exp \left[i q\left(x_{1}+\cdots+x_{n}\right)\right]$, there are other states with broken replica symmetry [21]. A treatment by Bouchaud and Orland [13] includes some of the effects of such excitations but is not fully rigorous. It does predict that the extent of transverse fluctuations grows as $t^{\zeta}$ with $\zeta=2 / 3$ as observed numerically [15, 16]. There is in fact a relation between the exponents $\zeta$ and $\omega$ which follows from simple physical considerations [15]: By analogy with a string, the energy to stretch a path by a distance $x$ grows as $x^{2} / t$. The path wanders away from the origin, only if the cost of this stretching can be made up by favorable configurations of bonds. Since the typical fluctuations in (free) energy at scale $t$ grow as $t^{\omega}$, we have

$$
\frac{x^{2}}{t} \propto t^{\omega} \quad \Longrightarrow \quad \omega=2 \zeta-1 \text {. }
$$


This relation remains valid in higher dimensions and has been verified in many numerical simulations. The first (indirect) proof of $\omega=1 / 3$ was based on a replica analysis of a problem with many interacting paths [22]. It was soon followed by a more direct proof [23] based on a completely different approach: the Cole-Hopf transformation described in next section.

\section{H. Higher Dimensions}

The approach described in the previous sections is easily generalized to higher dimensions. The directed path in $d=D+1$ is described by $\vec{x}(t)$, where $\vec{x}$ is a $D$ dimensional vector. The recursion relation of eq. (95) is generalized to

$$
W(\vec{x}, t+1)=\sum_{i=1}^{d} \tau_{\vec{x}-\vec{e}_{i}, t} W\left(\vec{x}-\vec{e}_{i}, t\right)
$$

where $\vec{e}_{i}$ are unit vectors. The recursion relation is easily iterated on a computer, but the memory requirement and execution time now grow as $t^{D}$ and $t^{D+1}$ respectively. The continuum limit of this recursion relation is

$$
\frac{\partial W(\vec{x}, t)}{\partial t}=-\frac{W}{\xi}+\nu \nabla^{2} W+\mu(\vec{x}, t) W
$$

where $\mu(\vec{x}, t)$ represents the fluctuations of $\tau(\vec{x}, t)$ around its average. Thus it has zero mean, and a variance

$$
\overline{\mu(\vec{x}, t) \mu\left(\vec{x}^{\prime}, t^{\prime}\right)}=\sigma^{2} \delta^{D}\left(\vec{x}-\vec{x}^{\prime}\right) \delta\left(t-t^{\prime}\right)
$$

(In a more general anisotropic situation, eq. (98) has to be generalized to include different diffusivities $\nu_{\alpha}$ along different directions. Such anisotropy is easily removed by rescaling the coordinates $x_{\alpha}$.)

Eq. (98) can be regarded as the imaginary time Schrödinger equation for a particle in a random time dependent potential. It can be integrated to yield the continuous path integral

$$
W(\vec{x}, t)=\int_{(0,0)}^{(\vec{x}, t)} \mathcal{D} \vec{x}\left(t^{\prime}\right) \exp \left[-\int_{0}^{t} d t^{\prime}\left(\frac{1}{\xi}+\frac{\dot{\vec{x}}^{2}}{4 \nu}-\mu\left(\vec{x}\left(t^{\prime}\right), t^{\prime}\right)\right)\right]
$$


describing the fluctuations of a directed polymer in a random medium (DPRM) [24]. The $n^{\text {th }}$ moment of $W$ is computed by replicating the above path integral and averaging over $\mu(\vec{x}, t)$. It generalizes eq. (73) to

$$
\begin{aligned}
W_{n}\left(\left\{\vec{x}_{\alpha}\right\}, t\right)= & \int_{(\{\overrightarrow{0}\}, 0)}^{\left(\left\{\vec{x}_{\alpha}\right\}, t\right)} \mathcal{D} \vec{x}_{1}\left(t^{\prime}\right) \cdots \mathcal{D} \vec{x}_{n}\left(t^{\prime}\right) \\
& \exp \left[-\int_{0}^{t} d t^{\prime}\left(\sum_{\alpha} \frac{1}{\xi}+\frac{\dot{\vec{x}}_{\alpha}^{2}}{4 \nu}-\frac{u}{2} \sum_{\alpha \neq \beta} \delta^{D}\left(\vec{x}_{\alpha}\left(t^{\prime}\right)-\vec{x}_{\beta}\left(t^{\prime}\right)\right)\right)\right]
\end{aligned}
$$

with $u \propto \sigma^{2}$. The differential equation governing the evolution of $W_{n}(t)$ is,

$$
\frac{\partial W_{n}}{\partial t}=-\frac{n}{\xi} W_{n}+\nu \sum_{\alpha} \nabla_{\alpha}^{2} W_{n}+\frac{u}{2} \sum_{\alpha \neq \beta} \delta^{D}\left(\vec{x}_{\alpha}-\vec{x}_{\beta}\right) W_{n} \equiv-H_{n} W_{n}
$$

Evaluating the asymptotic behavior of $W_{n}(t)$ requires knowledge of the ground state energy of the Hamiltonian $H_{n}$. Unfortunately, the exact dependence of the bound state energy on $n$ is known only for $D=0(\varepsilon \propto n(n-1))$ and $D=1\left(\varepsilon \propto n\left(n^{2}-1\right)\right)$. As discussed earlier, these two results can then be used to deduce the behavior of the bulk of the probability distribution for $\ln W(t)$. Elementary results from quantum mechanics tell us that an arbitrarily small attraction leads to the formation of a bound state in $D \leq 2$, but that a finite strength of the potential is needed to form a bound state in $D>2$. Thus, in the most interesting case of $2+1$ dimensions we expect a non-trivial probability distribution, while the replica analysis provides no information on its behavior. In higher dimensions, there is a phase transition between weak and strong randomness regimes. For weak randomness there is no bound state and asymptotically $\overline{W^{n}(t)}=\overline{W(t)}^{n}$, indicating a sharp probability distribution. This statement has also been established by more rigorous methods [25]. There is another phase for strong randomness where the probability distribution for $W(t)$ becomes broad. The resulting bound state has been analytically studied in a $1 / D$ expansion valid for large $D$ [26]. The ground state wavefunction is rather complex, involving replica symmetry breaking. Note that the phase transition in the probability distribution of the correlation function occurs in the high temperature phase of the random Ising model. The implications of this phase transition for bulk properties are not known. As the stiffness associated with line tension decreases on approaching the order/disorder phase transition of the Ising model, close to this transition the probability distribution for $W(t)$ is likely to be broad. 
As one of the simplest models of statistical mechanics in random systems (a "toy" spin glass), the problem of DPRM has generated considerable interest [27]. The model has been generalized to manifolds of arbitrary internal dimensions in random media [28], and treated by functional RG methods [29]. The same model has also been studied by a variational approach that involves replica symmetry breaking [30]. The latter is also exact in the $D \rightarrow \infty$ limit. Directed paths have been examined on non-Euclidean lattices: In particular, the problem can be solved exactly on the Cayley tree [31], where it has a transition between a "free" and a glassy state. There are also quite a few treatments based on a position space renormalization group scheme [32] which becomes exact on a hierarchical lattice [33]. This lattice has no loops, and at the $m+1^{\text {th }}$ level is constructed by putting together $2^{D}$ branches, each containing two lattices of the $m^{\text {th }}$ level. Starting from a set of random bonds at the first level, the values of the sum $W\left(m=\log _{2} t\right)$ are constructed recursively from

$$
W(m+1, \beta)=\sum_{\alpha=1}^{2^{D}} W\left(m, \alpha_{1}\right) W\left(m, \alpha_{2}\right)
$$

where the greek indices are used to indicate specific bonds for a particular realization. Alternatively, these recursion relations can be used to study the evolution of the probability distribution for $W$ [34]. The exponent $\omega \approx 0.30$ for $D=1$ is not too far off from the exact value of $1 / 3$.

Additional information about the higher dimensional DPRM is obtained by taking advantage of a mapping to the nonequilibrium problem of kinetic roughening of growing interfaces. Using the Cole-Hopf transformation [35],

$$
W(\vec{x}, t)=\exp \left[-\frac{\lambda h(\vec{x}, t)}{2 \nu}\right],
$$

eq. (98) is transformed to the Kardar, Parisi, Zhang (KPZ) [36] equation,

$$
\frac{\partial h}{\partial t}=\frac{2 \nu}{\lambda \xi}+\nu \nabla^{2} h-\frac{\lambda}{2}(\nabla h)^{2}-\frac{2 \nu}{\lambda} \mu(\vec{x}, t),
$$

describing the fluctuations in height $h(\vec{x}, t)$ of a growing interface. A dynamical renormalization group ( $\mathrm{RG}$ ) analysis at the one-loop level [37,38] of this equation indicates that the effective coupling constant $g=4 \sigma^{2} / \nu$, satisfies the rescaling relation

$$
\frac{d g}{d \ell}=(2-D) g+\frac{K_{D}(2 D-3)}{D} g^{2},
$$


where $K_{D}$ is the $D$ dimensional solid angle divided by $(2 \pi)^{D}$. The RG equation merely confirms the expectations based on the replica analysis: there is flow to strong coupling for $D \leq 2$, while there is a transition between weak and strong coupling behavior in higher dimensions. The RG equation has been recently extended to two loops [39,40]. According to one calculation [40, there is no stable strong coupling fixed point for $D \geq 2$, and the nature of this phase remains a mystery.

Since there are several comprehensive reviews of the KPZ equation [41], I will not discuss its properties in any detail here. It suffices to say that there are many numerical models of growth that fall in the universality class of this equation. They are in complete agreement with the exactly known results for $D=1$. The estimates for the exponent $\zeta$ in higher dimensions are $\zeta=0.624 \pm 0.001$ for $D=2$ 42] and $\zeta \approx 0.59$ for $D=3$ [43]. The numerical results in higher dimensions are consistent with an exponent $\zeta$ that gets closer to $1 / 2$ as $D \rightarrow \infty$. It is not presently known whether there is a finite upper critical dimension [28,44] beyond which $\zeta=1 / 2$ exactly.

\section{Random Signs}

So far we focused on nearest neighbor bonds $\left\{K_{i j}\right\}$, which though random, were all positive. For such couplings the ground state is uniform and ferromagnetic. The study of low temperature states is considerably more complicated for the random spin glass which describes a mixture of ferromagnetic and antiferromagnetic bonds. The competition between the bonds leads to frustration effects, resulting in quite complicated landscapes

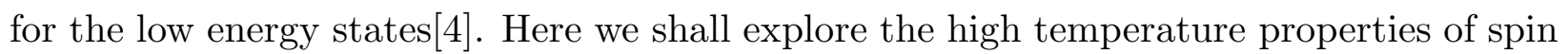
glass models. To focus on the effects of the randomness in sign, we study a simple binary probability distribution in which negative and positive bonds of equal magnitude occur with probabilities $p$ and $1-p$ respectively.

The computation of the high temperature series for the correlation function (along the diagonal) proceeds as before, and

$$
W(\vec{x}, t) \equiv\left\langle\sigma_{0,0} \sigma_{\vec{x}, t}\right\rangle=\tau^{t} \sum_{P} \prod_{i=1}^{t} \eta_{P i}
$$

where $\tau$ indicates the fixed magnitude of $\tanh K$, while $\eta_{P i}= \pm 1$ are random signs. Since the elements of the sum can be both positive and negative, the first question is whether the system maintains a coherence in sign (at least for small $p$ ), i.e. what is the likelihood 
that the two spins separated by a distance $t$ have a preference to have the same sign. This question can be answered only in one and high dimensions.

For the one dimensional chain the moments of $W(t)$ are easily calculated as

$$
\overline{W^{n}(t)}=\tau^{n t} \times \begin{cases}(1-2 p)^{t} & \text { for } n \text { odd } \\ 1 & \text { for } n \text { even }\end{cases}
$$

As all odd moments asymptotically decay to zero, at large distances $W(t)$ is equally likely to be positive or negative. This is because the sign of the effective bond depends only on the product of the intermediate bonds and the possibility of a few negative bonds is sufficient to remove any information about the overall sign. From eq. (108), we can define a characteristic sign correlation length $\xi_{s}=-1 / \ln (1-2 p)$.

There is also a "mean-field" type of approach to the sign coherence problem [45] which is likely to be exact in high dimensions. For paths along the diagonal of the hypercubic lattice, the mean value of $W(t)$ is

$$
\overline{W(t)} \approx[d \tau(1-2 p)]^{t}
$$

Calculating the variance of $W$ is complicated due to the previously encountered problem of intersecting paths. We can approximately evaluate it by considering a subset of paths contributing to the second moment as,

$$
\begin{aligned}
\overline{W^{2}} & \approx[d \tau(1-2 p)]^{2 t}+\left(d \tau^{2}\right)[d \tau(1-2 p)]^{2(t-1)}+\left(d \tau^{2}\right)^{2}[d \tau(1-2 p)]^{2(t-2)}+\cdots+\left(d \tau^{2}\right)^{t} \\
& =\tau^{2 t} \frac{[d(1-2 p)]^{2(t+1)}-d^{t+1}}{[d(1-2 p)]^{2}-d} .
\end{aligned}
$$

The first term in the above sum comes from two distinct paths between the end points; the second term from two paths that have their first step in common and then proceed independently. The $m^{\text {th }}$ term in the series describes two paths that take $m$ steps together before becoming separated. The underlying assumption is that once the two paths have separated they will not come back together again. This Independent Path Approximation (IPA) is better justified in higher dimensions and leads to

$$
\frac{\overline{W^{2}(t)}}{\overline{W^{2}(t)}}=\frac{d(1-2 p)^{2}-\left[d(1-2 p)^{2}\right]^{-t}}{d(1-2 p)^{2}-1} .
$$

For small $p$ such that $d(1-2 p)^{2}>1$, the above ratio converges to a constant as $t \rightarrow \infty$; the distribution is asymptotically sharp and the correlations preserve sign information. 
However, if the concentration of negative bonds exceeds $p_{c}=(1-1 / \sqrt{d}) / 2$, the ratio diverges exponentially in $t$, indicating a broad distribution. This has been interpreted [46] as signalling a "sign transition". This argument suggests that there is a finite $p_{c}$ for all $d>1$. However, it is important to note that the IPA ignores important correlations between the paths. Shapir and Wang [47] criticize the assumption of independent paths and suggest that as intersections are important for $d \leq 3$, there should be no phase transition in these dimensions. However, the identification of the lower critical dimension for the sign transition is not completely settled. Numerical simulations based on the transfer matrix method for $t$ of up to 600 [14], as well as exact enumeration studies [48] for $t \leq 10$, fail to find a phase transition in $d=2$. The results suggest that if there is a phase transition in $d=2$ it occurs for $p_{c}<0.05$. The phase diagram of a generalized model with complex phases has also been studied in higher dimensions [49,50].

For $p>p_{c}$ the information on sign is lost beyond a coherence length $\xi_{s}$. If the system is coarse grained beyond this scale, the effective bonds are equally likely to be positive or negative. Thus we shall concentrate on the symmetric case of $p=1 / 2$ in the rest of this section. This corresponds to the much studied $\pm J$ Ising spin glass [51 which will be discussed in more detail later on. We performed [14] transfer matrix computations on systems of up to size $t=2000$, and averaged over 2000 realizations of randomness, on a VaxStationII. The random numbers $(+1$ or -1$)$ were generated by a well tested random number generator [52]. Since $W$ grows exponentially in $t, \ln |W|$ has a well defined probability distribution; we examined its mean $\overline{\ln |W(t)|}$, and variance $\overline{\ln |W(t)|^{2}}-\overline{\ln |W(t)|^{2}}$, for $p=1 / 2$ (both signs equally probable). We also computed the typical excursions of the paths in the lateral direction as defined by

$$
\overline{\left[x(t)^{2}\right]_{a v}} \equiv \frac{\overline{\sum_{x} x^{2}|W(x, t)|^{2}}}{\sum_{x}|W(x, t)|^{2}},
$$

and

$$
\overline{[x(t)]_{a v}^{2}} \equiv \overline{\left(\frac{\sum_{x} x|W(x, t)|^{2}}{\sum_{x}|W(x, t)|^{2}}\right)^{2}},
$$

where $[\cdot]_{a v}$ denotes an average over the lateral coordinate at a fixed $t$, using a weight $|W(x, t)|^{2}$.

We confirmed that the average of $\ln |W(t)|$ is extensive $(\overline{\ln |W(t)|}=(0.322 \pm 0.001) t)$, while its fluctuations satisfy a power law growth $t^{\omega}$, with $\omega=0.33 \pm 0.05$. For several choices of $t$ we also checked in detail that $W(t)$ is positive or negative with equal probability. 
For lateral excursions, we examined simulations with $t=4000$, and with 200 realizations of randomness (reasonable data for fluctuations of $\ln |W(t)|$ are only obtained from higher averaging). The results for $\overline{\left[x^{2}\right]_{a v}}$ and $\overline{[x]_{a v}^{2}}$ appear to converge to a common asymptotic limit; fitted to a power law $t^{2 \zeta}$ with $\zeta=0.68 \pm 0.05$. The scaling properties of $|W(x, t)|$ thus appear identical to those of directed polymers with positive random weights! It should be noted, however, that using a similar procedure, Zhang [53] concluded from fits to his numerical results a value of $\zeta=0.74 \pm 0.01$. Using a variety of theoretical arguments [53], he suggests $\omega=1 / 2$ and $\zeta=3 / 4$. The exponent $\omega=1 / 2$ is clearly inconsistent with our data, while $\zeta=3 / 4$ can be obtained if one fits only to $\overline{[x]_{a v}^{2}}$. Two subsequent, rather extensive, numerical studies [54,55] shed more light on this problem. Both simulations seem to equivocally point to the importance of including corrections to scaling in the fits. In $1+1$ dimensions they indeed find $\omega=1 / 3$ for the variance, and $\zeta=2 / 3$ (with a large correction to scaling term) for transverse fluctuations.

The similarity in the probability distributions of random weight and random sign problems can be understood by examination of the moments. The terms in $W^{n}$ correspond to the product of contributions from $n$ independent paths. Upon averaging, if $m$ paths cross a particular bond $(0 \leq m \leq n)$, we obtain a factor of $\left[1+(-1)^{m}\right] / 2$, which is 0 or 1 depending on the parity of $m$. For odd $n$ there must be bonds with $m$ odd, and hence $\overline{W^{2 n+1}}=0$; which of course implies and follows from the symmetry $p(W)=p(-W)$. For even moments $\overline{W^{2 n}}$, the only configurations that survive averaging are those in which the $2 n$ replicated paths are arranged such that each bond is crossed an even number of times. The simplest configurations satisfying this constraint correspond to drawing $n$ independent paths between the end points and assigning two replica indices to each. The above constraint is also satisfied by forming groups of four or higher even numbers, but such configurations are entropically unlikely and we shall henceforth consider only paired paths. There is an important subtlety in calculating $\overline{W^{2 n}}$ from the $n$ paired-paths: After two such paths cross, the outgoing pairs can either carry the same replica labels as the ingoing ones, or they can exchange one label (e.g. $(12)(34) \rightarrow(12)(34),(13)(24)$, or $(14)(23))$. Therefore, after summing over all possible ways of labelling the paired paths, there is a multiplicity of three for each intersection. The $n$ paired paths attract each other through the exchange of replica partners! 
Although the origin of the attraction between paths is very different from the case of random weights, the final outcome is the same. The even moments in $1+1$ dimension are related by an expression similar to eq. (89),

$$
\lim _{t \rightarrow \infty} \overline{W^{2 n}(t)}={\overline{W(t)^{2}}}^{n} \exp \left[\rho n\left(n^{2}-1\right) t\right]
$$

and the conclusions regarding $\ln W(t)$ are the same as before. If, rather than having only one possible value for the magnitude of the random bond, we start with a symmetric distribution $p(\tau)$, there will be an additional attraction between the paired paths coming from the variance of $\tau^{2}$. This increases the bound state energy (and the factor $\rho$ ) in eq. (114) but does not affect the universal properties.

\section{J. Other Realizations of DPRM}

So far we focused on sums over DPRM as encountered in high temperature series of Ising models. In fact several other realizations of such paths have been discussed in the literature, and many more are likely to emerge in the future. One of the original motivations was to understand the domain wall of an Ising model in the presence of random bond impurities [15]. As mentioned in the previous section, if all the random bonds are ferromagnetic, in the ground state all spins are up or down. Now consider a mixed state in which a domain wall is forced into the system by fixing spins at opposite edges of the lattice to + and - . Bonds are broken at the interface of the two domains, and the total energy of the defect is twice the sum of all the $K_{i j}$ crossed by the interface. In the Solid-On-Solid approximation, configurations of the domain wall are restricted to directed paths. The resulting partition function $Z(t)$, can be computed by exactly the same transfer matrix method used to calculate $W(t)$. Rather than looking at the finite temperature partition function, Huse and Henley [15] worked directly with the zero temperature configuration of the interface.

Denoting by $E(x, t)$ the minimum in the energy of all paths connecting $(0,0)$ to $(x, t)$, oriented along the diagonal of the square lattice, it is possible to construct the recursion relation,

$$
E(x, t+1)=\min \left\{E(x-1, t)-2 J_{x-1, t}, E(x+1, t)-2 J_{x+1, t}\right\} \quad,
$$

closely related to eq. (95). To find the actual configuration of the path, it is also necessary to store in memory one bit of information at each point $(x, t)$, indicating whether the 
minimum in eq. (115) comes from the first or second term. This bit of information indicates the direction of arrival for the optimal path at $(x, t)$. After the recursion relations have been iterated forward to "time" step $t$, the optimal location is obtained from the minimum of the array $\{E(x, t)\}$. From this location the optimal path is reconstructed by stepping backward along the stored directions. This is how the pictures of optimal paths in refs. [24, 38] were constructed. These optimal paths have a beautiful ultrametric structure that resembles the deltas of river basins, and many other natural branching patterns. Finding the optimal interface is reminiscent of the travelling salesman problem. However, in this case, although the number of possible paths grow as $2^{t}$, their directed nature allows us to find the best solution in polynomial time.

The statistics of the $E(x, t)$ at $T=0$ are identical to those of $\ln W(x, t)$ : the optimal path wanders as $t^{2 / 3}$, while the fluctuations in $E(t)$ scale as $t^{1 / 3}$ [15]. It is frequently assumed that these fluctuations also set the scale of energy barriers that the interface must cross from one optimal state to another. Since such barriers grow with $t$, any activated process is slowed down to a logarithmic crawl [15].

It has been suggested that optimal paths are also relevant to fracture and failure phenomena [56]. Imagine a two dimensional elastic medium with impurities, e.g. a network of springs of different strengths and extensions [57]. If the network is subjected to external shear, a complicated stress field is set up in the material. It is possible that non-linear effects in combination with randomness enhance the stress field along particular paths in the medium. Such bands of enhanced stress are visible by polarized light in a sheet of plexiglass. The localization of deformation is nicely demonstrated in a two dimensional packing of straws [58]. The roughness of the localization band is characterized by the exponent $\zeta=0.73 \pm 0.07$, not inconsistent with the value of $2 / 3$ for DPRM. The experiment was inspired by random fuse models [59] which apply a similar procedure to describe the failure of an electrical network. Hansen et al. [60] suggest that at the threshold in all such models, failure occurs along an optimal path with statistics similar to a DPRM. Their numerical results obtain a roughness exponent of $\zeta=0.7$ for the crack interface with a precision of about $10 \%$.

In fact, the minimal directed path was proposed in 1964 [61] as a model for tensile rupture of paper. The variations in brightness of a piece of paper held in front of a light source are indicative of nonuniformities in local thickness and density $\rho(\mathbf{x})$. Tydeman and Hiron suggested that rupture occurs along the weakest line for which the sum of $\rho(\mathbf{x})$ is minimum. This is clearly just a continuum version of the optimal energy path in a random 
medium. (Since the average of $\rho(\mathbf{x})$ is positive, the optimal path will be directed.) This model was tested by Kertész et al. 62 who used a tensile testing machine to gradually tear apart many sheets of paper. They found that the resulting rupture lines are self-affine, characterized by $0.63<\zeta<0.72$.

The three dimensional DPRM was introduced [24] as a model for a polyelectrolyte in a gel matrix. Probably a better realization is provided by defect lines, such as dislocations or vortices, in a medium with impurities. There has been a resurgence of interest in this problem since it was realized that flux lines in high temperature ceramic superconductors are highly flexible, and easily pinned by the oxygen impurities that are usually present in these materials [63]. Pinning by impurities is in fact crucial for any application, as otherwise the flux lines drift due to the Lorentz force giving rise to flux flow resistivity [64.

\section{K. Quantum Interference of Strongly Localized Electrons}

The wavefunctions for non-interacting electrons in a regular solid are extended Bloch states. In the presence of disorder and impurities, gradually more and more of these states become localized. This was first pointed out by Anderson 65 who studied a random tight-binding Hamiltonian

$$
\mathcal{H}=\sum_{i} \varepsilon_{i} a_{i}^{+} a_{i}+\sum_{<i j>} V_{i j} a_{i}^{+} a_{j}
$$

Here $\varepsilon_{i}$ are the site energies and $V_{i j}$ represent the nearest neighbor couplings or transfer terms. For simplicity we shall focus on

$$
V_{i j}= \begin{cases}V & \text { if } i, j \text { are nearest neighbors } \\ 0 & \text { otherwise }\end{cases}
$$

so that all the randomness is in the site energies. This is just a discretized version of the continuum Hamiltonian $H=\nu \nabla^{2}+\varepsilon(\vec{x})$, for a quantum particle in a random potential $\varepsilon(\vec{x})$. For a uniform $\varepsilon$, the Hamiltonian is diagonalized by extended Fourier modes $a_{\vec{q}}^{\dagger}=$ $\sum_{\vec{x}} \exp (i \vec{q} \cdot \vec{x}) a_{\vec{x}}^{\dagger} / \sqrt{N}$, resulting in a band of energies $\varepsilon(\vec{q})=\varepsilon+2 V\left(\cos q_{1}+\cos q_{2}+\cdots+\right.$ $\cos q_{d}$ ). (The lattice spacing has been set to unity.) As long as the fermi energy falls within this band of excited states the system is metallic.

In the random system the wave functions become distorted, and localized to the vicinity of low energy impurities 65. This localization starts with the states at the edge 
of the band and proceeds to include all states as randomness is increased. In fact in $d \leq 2$, as the diffusing path of a non-localized electron will always encounter an impurity, all states are localized by even weak randomness. The original ideas of Anderson localization [65], and a heuristic scaling approach by Thouless [66], have been placed on more rigorous footing by perturbative RG studies [67 69]. The perturbative approach emphasizes the importance of quantum interference effects in the weakly disordered metal. Weak localization phenomena include the effects of magnetic fields, spin-orbit (SO) scattering (corresponding respectively to interactions breaking time reversal and spin space symmetries) on the conductivity [70], as well as predicting a universal value of the order of $e^{2} / \hbar$ for conductance fluctuations [71,72. These phenomena can be traced to the quantum interference of time reversed paths in backscattering loops and their suppression by magnetic fields and SO [73]: In the of absence SO, a magnetic field causes an increase in the localization length, and a factor of 2 decrease in the conductance fluctuations; with SO, it has the opposite effect of decreasing the localization length, while still reducing the conductance fluctuations [74, 75]. An alternative description of these phenomena is based on the theory of random matrices [76], where the only input is the symmetries of the underlying Hamiltonian and their modification by a magnetic field. Mesoscopic devices at low temperature have provided many experimental verifications of weak localization theory 73,77 and there are many excellent reviews on the subject [70,78].

When the electronic states at the fermi energy are localized, the material is an insulator and there is no conductivity at zero temperature. However, at finite temperatures there is a small conductivity that originates from the quantum tunneling of electrons between localized states, described by Mott's variable range hopping (VRH) process [79]: The probability for tunneling a distance $t$ is the product of two factors

$$
p(t) \propto \exp \left(-\frac{2 t}{\xi}\right) \times \exp \left(-\frac{\delta \varepsilon}{k_{B} T}\right) .
$$

The first factor is the quantum tunneling probability and assumes that the overlap of the two localized states decays with a characteristic localization length $\xi$. The second factor recognizes that the different localized states must have different energies $\delta \varepsilon$ (otherwise a new state is obtained by their mixture using degenerate perturbation theory). The difference in energy must be provided by inelastic processes such as phonon scattering, and is governed by the Boltzmann weight at temperature $T$. The most likely tunneling sites must be close in energy. If there is a uniform density of states $N\left(\varepsilon_{f}\right)$ in the vicinity 
of the fermi energy, there are roughly $N\left(\varepsilon_{f}\right) t^{d}$ candidate states in a volume of linear size $t$ in $d$ dimensions, with the smallest energy difference of the order of $\delta \varepsilon \propto\left(N\left(\varepsilon_{f}\right) t^{d}\right)^{-1}$. Thus the two exponential factors in eq. (117) oppose each other, encouraging the electron to travel shorter and longer distances respectively. The optimal distance scales as

$$
t \approx \xi\left(T_{0} / T\right)^{\frac{1}{d+1}}
$$

with $T_{0} \propto\left(k_{B} N\left(\varepsilon_{f}\right) \xi^{d}\right)^{-1}$, diverging at zero temperature.

In the strongly localized regime, the optimal hopping length is many times greater than the localization length $\xi$. The localized sites are then assumed to be connected by a classical random resistor network [80]. Since the individual resistors are taken from a very wide distribution, it is then argued [81] that the resistance of the whole sample is governed by the critical resistor that makes the network percolate. This leads to a dependence

$$
\sigma(T)=\sigma_{0} \exp \left[-\left(T_{0} / T\right)^{\frac{1}{d+1}}\right]
$$

for the conductivity. This behavior has been verified experimentally both in two and three dimensions [82]. Due to the difficulty of measuring variations in the much smaller conductivities of insulators, there have been relatively few studies of the conductivity and its fluctuations for strongly localized electrons. Nonetheless, recent experiments [83] find a positive $\mathrm{MC}$ in $\mathrm{Si}$-inversion layers, $\mathrm{GaAs}$ and $\mathrm{In}_{2} \mathrm{O}_{3-x}$ films. Furthermore, the observed reproducible conductance fluctuations are quite suggestive of quantum interference (QI) effects. However, the magnitudes of these fluctuations grow with lowering temperature, and are about 100 times larger than $e^{2} / \hbar$ at the lowest temperature.

Clearly a different theory is needed to account for QI effects in the strong localization regime. The most natural candidate is the quantum overlap factor in eq. (117). Nguyen, Spivak, and Shklovskii (NSS) have proposed a model that accounts for QI of multiply scattered tunneling paths in the hopping probability: In between the phonon assisted tunneling events the electron preserves its phase memory. However, at low temperatures it tunnels over very large distances according to eq. (118), and encounters many impurities. The overall tunneling amplitude is then obtained from the sum over all trajectories between the initial and final sites. NSS emphasized that since the contribution of each trajectory is exponentially small in its length, the dominant contributions to the sum come from the shortest or forward scattering paths. The traditional explanations of weak localization phenomena which rely on the QI of back scattering paths are therefore inappropriate to this regime. This picture is clearly reminiscent of the directed paths and will be developed more formally in the next section. 


\section{The Locator Expansion and Forward Scattering Paths}

The overlaps in the insulating regime can be studied by performing a "locator" expansion [65]; valid in the limit $\left|V_{i j}\right|=V \ll\left(E-\varepsilon_{i}\right)$, where $E$ is the electron energy. Indeed, for $V=0$, the eigenfunctions are just the single site states, and the localization length is zero (no transfer term). For $V /\left(E-\varepsilon_{i}\right) \ll 1$, various quantities can be obtained perturbatively around this solution, as expressed by the Lippman-Schwinger equation 84.

$$
\left|\Psi^{+}\right\rangle=|\Phi\rangle+\frac{1}{E-H_{0}+i \delta} \mathcal{V}\left|\Psi^{+}\right\rangle
$$

The bare Hamiltonian

$$
H_{0}=\sum_{i} \varepsilon_{i} a_{i}^{+} a_{i}
$$

has no nearest-neighbor coupling, while the perturbation

$$
\mathcal{V}=\sum_{<i j>} V_{i j} a_{i}^{+} a_{j}
$$

describes the small transfer terms. $|\Phi\rangle$ represents the state with a localized electron at the initial site (or incident wave), $\left|\Psi^{+}\right\rangle$the state where a localized electron is at the final site. In the coordinate representation, the wavefunctions are exponentially localized around the impurity sites and there are no propagating waves since electrons can only tunnel under a potential barrier. (This situation was first addressed in detail by Lifshits and Kirpichenko 855.) We can now iterate this implicit equation to obtain an expansion in powers of the ratio $\mathcal{V} /\left(E-\varepsilon_{i}\right)$ as

$$
\left|\Psi^{+}\right\rangle=|\Phi\rangle+\frac{1}{E-H_{0}+i \delta} \mathcal{V}|\Phi\rangle+\frac{1}{E-H_{0}+i \delta} \mathcal{V} \frac{1}{E-H_{0}+i \delta} \mathcal{V}|\Phi\rangle+\cdots .
$$

Acting with $\left\langle\Psi^{+}\right|$on the left and taking $\delta$ to zero, we obtain the overlap between the two states

$$
\left\langle\Psi^{+} \mid \Psi^{+}\right\rangle=\left\langle\Psi^{+} \mid \Phi\right\rangle+\left\langle\Psi^{+}\left|\frac{1}{E-H_{0}} \mathcal{V}\right| \Phi\right\rangle+\left\langle\Psi^{+}\left|\frac{1}{E-H_{0}} \mathcal{V} \frac{1}{E-H_{0}} \mathcal{V}\right| \Phi\right\rangle+\cdots
$$

For a more general transfer term $\mathcal{V}$ connecting all sites, the first term represents an electron starting from the initial site and ending at the final site without scattering (the overlap $\left.\left\langle\Psi^{+} \mid \Phi\right\rangle\right)$; the second term represents electrons scattering once off intermediate sites, the third, scattering twice, etc.. The operator $\mathcal{V}$ acting on $|\Phi\rangle$ produces a factor $V$ for each segment crossed, and $H_{0}$ acting on a particular site $i$ results in $\varepsilon_{i}$, the bare site energy. 
Thus we finally arrive at a simple expression for the amplitude or the Green's function between the initial and final states as

$$
\left\langle\Psi^{+} \mid \Psi^{+}\right\rangle=\left\langle\Phi|G(E)| \Psi^{+}\right\rangle=V \sum_{\Gamma} \prod_{i_{\Gamma}} \frac{V}{E-\varepsilon_{i_{\Gamma}}} .
$$

The terms in the above perturbation series correspond to all paths $\Gamma$ connecting the end points; $i_{\Gamma}$ label the sites along each path. Except that the random variables appear on the sites rather than the bonds of the lattice, this sum over paths is quite reminiscent of the corresponding one for the correlation functions of the random bond Ising model. There is, however, one complication that distinguishes the localization problem: The energy denominators in eq. (123) may accidentally be zero, invalidating the perturbation series. Physically, this corresponds to intermediate sites that are at the same energy as the external points. Presumably in this case a degenerate perturbation theory has to be used to construct the wavefunction. NSS [46] circumvent this issue by considering initial and final sites of approximately the same energy $\varepsilon_{F}=E=0$, while the intermediate sites have energies $\varepsilon_{i}= \pm U$ with equal probability. All the energy denominators in eq. (123) now contribute the same finite magnitude $U$, but random signs $\eta_{i_{\Gamma}}=\varepsilon_{i_{\Gamma}} / U$. The justification is that the Mott argument implicitly assumes that the lowest energy $\delta \varepsilon$ occurs at a distance $t$, and that there are no intermediate sites that are more favorable. However, it is not clear that due to the very same considerations, we should not include some dependence of the effective energy gap $U$ on $t$. We shall set aside such considerations and focus on the properties of the NSS model in the remainder.

A path of length $\ell$ now contributes an amplitude $U(V / U)^{\ell}$ to the sum, as well as an overall sign. In the localized regime the sum is rapidly convergent, dominated by its lowest order terms 65]. In general, the sum is bounded by one in which all terms make a positive contribution, i.e. by a lattice random walk which is convergent for $z(V / U)<1$, where $z$ is the lattice coordination number. This provides a lower bound for the delocalization transition, and the series is certainly convergent for smaller values of $V / U$. As in the Ising model we expect loops to become important only after the transition, while in the localized phase typical paths are directed beyond the localization length $\xi$. For $(V / U) \ll 1$, the localization length is less than a single lattice spacing, and only directed (forward scattering) paths need to be considered. Loops (back scattering paths) are irrelevant in the 
renormalization group sense. For sites separated by a distance $t+1$ along a diagonal of the square lattice, eq. (123) is now simplified to

$$
\langle i|G(E)| f\rangle=V\left(\frac{V}{U}\right)^{t} \sum_{P} \prod_{i=1}^{t} \eta_{P i}
$$

which is identical to eq. (107) with $(V / U)$ replacing $\tau$. The diagonal geometry maximizes possible interference by having a large number of shortest paths. For tunneling along the axes rather than the diagonal of a square lattice there is only one shortest path. Then, including longer paths with kinks is essential to the interference phenomena. However, the analogy to previous results suggests that the universal behavior is the same in the two cases while the approach to asymptotic behavior is much slower in the latter.

Using the equivalence to eq. (114), in conjunction with eq. (93), results in

$$
\lim _{t \rightarrow \infty} \overline{\ln |\langle i|G| f\rangle|^{2}}=\ln \left[2\left(\frac{V}{U}\right)^{2}\right] t-\rho t \equiv-2 t\left(\xi_{0}^{-1}+\xi_{g}^{-1}\right)
$$

where we have defined local and global contributions to the effective localization length, respectively given by

$$
\xi_{0}=\left[\ln \left(\frac{U}{\sqrt{2} V}\right)\right]^{-1} \quad, \quad \text { and } \quad \xi_{g}^{-1}=\frac{\rho}{2}
$$

The QI information is encoded in $2 \xi_{g}^{-1}=\rho$. Numerical estimates indicate that for the NSS model $\xi_{g} \approx 40$, and confirm that the width of the distribution scales as

$$
\delta \ln |\langle i|G| f\rangle| \sim\left|\frac{t}{\xi_{g}}\right|^{1 / 3}
$$

Since $t \propto T^{-1 / 3}$ in Mott VRH, we expect fluctuations in log-conductivity to grow as $T^{-1 / 9}$ for $T \rightarrow 0$, in qualitative agreement with the experimental results of ref. 83. (A quantitative test of this dependence has not yet been performed.)

\section{Magnetic Field Response}

All that is needed to include a magnetic field $B$ in the tight binding Hamiltonian of eq. (116) is to multiply the transfer elements $V_{i j}$ by $\exp \left(A_{i j}\right)$, where $A_{i j}$ is the line integral of the gauge field along the bond from $i$ to $j$. Due to these factors, the Hamiltonian becomes 
complex and is no longer time reversal symmetric $\left(H^{*} \neq H\right)$. In the parlance of random matrix theory [76], the Hamiltonian with $B=0$ belongs to the orthogonal ensemble, while a finite field places it in the unitary ensemble. Actually, random matrix theory recognizes a third (symplectic) ensemble of Hamiltonians which are time reversal symmetric, but not invariant under rotations in spin space. Up to this point we had not mentioned the spin of the electron: The states of eq. (116) are thus doubly degenerate and can be occupied by (non-interacting) up or down spin states. We can remove this degeneracy by including spin-orbit (SO) scattering, which rotates the spin of the electron as it moves through the lattice.

The generalized tight binding Hamiltonian that includes both the effects of SO scattering and magnetic field is

$$
H=\sum_{i, \sigma} \varepsilon_{i} a_{i, \sigma}^{\dagger} a_{i, \sigma}+\sum_{<i j>, \sigma \sigma^{\prime}} V_{i j, \sigma \sigma^{\prime}} e^{i A_{i j}} a_{i, \sigma}^{\dagger} a_{j, \sigma^{\prime}}
$$

The constant, nearest-neighbor only hopping, elements $V$ in eq. (116) are no longer diagonal in spin space. Instead, each is multiplied by $\mathcal{U}_{i j}$, a randomly chosen $S U(2)$ matrix which describes the spin rotation due to strong SO scatterers on each bond [76]. Eq. (123) for the overlap of wavefunctions at the two end-points must now include the initial and final spins, and eq. (124) for the sum of directed paths generalizes to

$$
\mathcal{A}=\left\langle i \sigma|G(0)| f \sigma^{\prime}\right\rangle=V(V / U)^{t} J(t) \quad ; \quad J(t)=\sum_{P} \prod_{j=1}^{t} \eta_{P j} e^{i A_{P j, P(j+1)}} \mathcal{U}_{P j, P(j+1)}
$$

After averaging over the initial spin, and summing over the final spin, the tunneling probability is

$$
T=\frac{1}{2} \operatorname{tr}\left(\mathcal{A}^{\dagger} \mathcal{A}\right)=V^{2}(V / U)^{2 t} I(t) \quad ; \quad I(t)=\frac{1}{2} \operatorname{tr}\left(J^{\dagger} J\right) \quad .
$$

We numerically studied the statistical properties of $I(t)$, using a transfer matrix method to exactly calculate $I$ up to $t=1000$, for over 2000 realizations of the random Hamiltonian. We again found that the distribution is broad (almost log-normal), and that the appropriate variable to consider is $\ln I(t)$. In all cases the mean of $\ln I(t)$ scaled linearly with $t$, while its fluctuations scaled as $t^{\omega}$ with $\omega \approx 1 / 3$ [86 88. For the sake of comparison with experiments we define a log-magnetoconductance (MC) by

$$
M C(t, B) \equiv \overline{\ln I(t, B)}-\overline{\ln I(t, 0)}
$$


We find numerically that the magnetic field always causes an enhancement in tunneling (a positive MC), but that the asymptotic behavior is quite distinct in the presence or absence of SO scattering.

(1) In the absence of $\mathrm{SO}$, the $\mathrm{MC}$ is unbounded and grows linearly with $t$. This can be interpreted as an increase in the global contribution to the localization length. The numerical results indicate that for small $B$, the change in slope is proportional to $B^{1 / 2}$. Indeed the data for different $t$ and $B$ can be collapsed together, using the fit

$$
M C(t, B)=(0.15 \pm 0.03)\left(\frac{\phi}{\phi_{0}}\right)^{1 / 2} t
$$

where $\phi=B a^{2}$ is the flux per plaquette, and $\phi_{0}$ is the elementary flux quantum.

(2) In the presence of $\mathrm{SO}$, the MC quickly saturates with $t$ and there is no change in the localization length. The data can still be collapsed, but by using $B t^{3 / 2}$ as the scaling argument, and we find

$$
M C_{S O}(t, B)=\left\{\begin{array}{ll}
c B^{2} t^{3} & \text { if } B^{2} t^{3}<1 \\
C \approx 0.25 & \text { if } B^{2} t^{3}>1
\end{array} .\right.
$$

We can gain some analytic understanding of the distribution function for $I(t, B)$ by examining the moments $\overline{I(t)^{n}}$. From eqs. (129) and (130) we see that each $I(t)$ represents a forward path from $i$ to $f$, and a time reversed path from $f$ to $i$. For $\overline{I(t)^{n}}$, we have to average over the contributions of $n$ such pairs of paths. Averaging over the random signs of the site energies forces a pairing of the $2 n$ paths (since any site crossed by an odd number of paths leads to a zero contribution) [86]. To understand the MC, it is useful to distinguish two classes of pairings: (1) Neutral paths in which one member is selected from $J$ and the other from $J^{\dagger}$. Such pairs do not feel the field since the phase factors of $e^{i A}$ picked up by one member on each bond are canceled by the conjugate factors $e^{-i A}$ collected by its partner. (2) Charged paths in which both elements are taken from $J$ or from $J^{\dagger}$. Such pairs couple to the magnetic field like particles of charge $\pm 2 e$. In the presence of $\mathrm{SO}$, we must also average over the random $S U(2)$ matrices. From the orthogonality relation for group representations [89], we have

$$
\int \Gamma^{k}(g)_{i j}^{*} \Gamma^{k^{\prime}}(g)_{i^{\prime} j^{\prime}} W\left(\alpha_{1}, \cdots, \alpha_{n}\right) d \alpha_{1} \cdots d \alpha_{n}=\frac{\delta_{i i^{\prime}} \delta_{j j^{\prime}} \delta_{k k^{\prime}}}{\lambda_{k}} \int W\left(\alpha_{1}, \cdots, \alpha_{n}\right) d \alpha_{1} \cdots d \alpha_{n},
$$

where $\Gamma^{k}(g)_{i j}$ is the $i j$ matrix element of a representation of the group element $g$, $W\left(\alpha_{1}, \cdots, \alpha_{n}\right)$ is an appropriate weight function so that the matrix space is sampled 
uniformly as the continuous parameters $\alpha_{1}, \cdots, \alpha_{n}$ vary (e.g. Euler angles for a representation of $S U(2))$. Finally $\lambda_{k}$ is the order of the representation $k$. Choosing the Euler angle parametrization of $S U(2)$ it can be shown that the only nonzero paired averages are

$$
\overline{\mathcal{U}_{\alpha \beta} \mathcal{U}_{\alpha \beta}^{*}}=\frac{1}{2} \quad, \quad \overline{\mathcal{U}_{\uparrow \uparrow} \mathcal{U}_{\downarrow \downarrow}}=\frac{1}{2} \quad, \quad \overline{\mathcal{U}_{\uparrow \downarrow} \mathcal{U}_{\downarrow \uparrow}}=-\frac{1}{2}
$$

and their complex conjugates. Thus SO averaging forces neutral paths to carry parallel spins, while the spins on the two partners of charged paths must be antiparallel.

We next consider the statistical weights associated with the intersections of paths. These weights depend crucially on the symmetries of the Hamiltonian in eq. (128): For $B=0$ and without SO, the Hamiltonian has orthogonal symmetry. All pairings are allowed and the attraction factor is 3 , since an incoming (12)(34) can go to (12)(34), (13)(24), or $(14)(23)$. Note that even if both incoming paths are neutral, one of the exchanged configurations is charged. A magnetic field breaks time reversal symmetry, discourages charged configurations, and reduces the exchange attraction. The limiting case of a 'large' magnetic field is mimicked by replacing the gauge factors with random phases. In this extreme, the Hamiltonian has unitary symmetry and only neutral paths are allowed. The exchange factor is now reduced to 2 ; from $\left(11^{*}\right)\left(22^{*}\right) \rightarrow\left(11^{*}\right)\left(22^{*}\right)$, or $\left(12^{*}\right)\left(21^{*}\right)$. With SO averaging, we must also take into account the allowed spin exchanges: Two neutral paths entering the intersection can have indices $(\alpha \alpha),(\alpha \alpha)$ or $(\alpha \alpha),(\overline{\alpha \alpha})$; there are 2 possibilities for the first $(\alpha=\uparrow$ or $\downarrow$ ) and two for the second $(\bar{\alpha})$ is antiparallel to $\alpha$ ). In the former case, however, there are two exchanges preserving neutrality, while in the latter only one exchange is possible satisfying this constraint. Hence an overall multiplicity of $[2 \times 2+2 \times 1] \times(1 / 2)^{2}=3 / 2$ is obtained, where the $(1 / 2)^{2}$ comes from the averages in eq. (135). Thus the intersection of two paired paths results in an exchange attraction of $3 / 2$; a signature of the symplectic symmetry.

Based on the above symmetry dependent statistical attraction factors, we can provide an understanding of the numerical results for MC. The sum over $n$ attracting paths again leads to

$$
\left\langle I(t)^{n}\right\rangle=A(n) 2^{n t} \exp \left[\rho n\left(n^{2}-1\right) t\right]
$$

where we have also included an overall $n$-dependent amplitude. Without SO, the magnetic field gradually reduces the attraction factor from 3 to 2 leading to the increase in slope. Addition of SO to the Hamiltonian has the effect of suddenly decreasing the attraction factor to $3 / 2$. Why does the addition of the magnetic field lead to no further change in $\rho$ in 
the presence of SO? Without SO, the origin of the continuous change in the attraction factor is a charged bubble that may appear in between successive intersections of two neutral paths. In the presence of SO, from the averages in eq. (135) we find the contribution of such configurations to be zero. To produce intermediate charged paths (with their antiparallel spins), the entering pair must have indices of the type $(i i),(\bar{i} \bar{i})$ (where $\bar{\downarrow}=\uparrow, \quad$ and $\quad \bar{\uparrow}=\downarrow$ ). Within the bubble we can have intermediate sites labeled $(j \bar{j})$ and $(k \bar{k})$ which must be summed over due to matrix contractions. It is easy to check that, independent of the choice of $j$, if the incoming and outgoing spins $(i$ and $m$ ) are the same on a branch it contributes a positive sign, while if they are opposite the overall sign is negative. However, for any choice of $i$ and $m$, one may choose similar (e.g. $i \rightarrow m$ on both branches), or opposite (e.g. $i \rightarrow m$ on top and $i \rightarrow \bar{m}$ on lower branch) connections. The difference in sign between the two choices thus cancels their overall contributions. Hence the neutral paths traverse the system without being affected by charged segments. In a magnetic field, their attraction factor stays at $3 / 2$ and $\rho=\xi_{g}^{-1}$ is unchanged.

The smaller positive MC observed in the simulations is due to changes in the amplitude $A(n)$ in eq. (136). This originates from the charged paths that contribute to tunneling at small $B$ but are quenched at higher $B$. However, due to their lack of interactions, we may treat the charged and neutral paths as independent. At zero field any of the pairings into charged and neutral paths is acceptable, while at finite fields only neutral pairs survive. This leads to a reduction in the amplitude $A(n)$ for $n \geq 2$, but an increase in $\ln I$ (a positive MC). The typical value of $\ln I$ thus increases by a $t$ independent amount. This behavior is similar to the predictions of IPA, and is indeed due to the independence of charged and neutral paths. Since the typical scale of decay for charged paths depends on the combination $B t^{3 / 2}$ (typical flux through a random walk of length $t$ ), we can explain the scaling obtained numerically in eq. (133).

The exchange attraction between neutral paths can also be computed for (unphysical) $S U(n)$ impurities and equals $1+1 / n$, which reproduces 2 for $U(1)$ or random phases, and $3 / 2$ for $S U(2)$ or $\mathrm{SO}$ scattering. The attraction vanishes in the $n \rightarrow \infty$ limit, where the paths become independent. The statistical exchange factors are thus universal numbers, simply related to the symmetries of the underlying Hamiltonian. The attractions in turn are responsible for the formation of bound states in replica space, and the universal scaling of the moments in eq. (136). In fact, since the single parameter $\rho$ completely characterizes the distribution, the variations in the mean and variance of $\ln I(t)$ should be perfectly correlated. This can be tested numerically by examining respectively coefficients of the 
mean and the variance for different cases. All results do indeed fall on a single line, parametrized by $\rho$. The largest value corresponds to the NSS model for $B=0$ and no SO (orthogonal symmetry, exchange attraction 3). Introduction of a field gradually reduces $\rho$ until saturated at the limit of random phases (unitary symmetry, exchange attraction 2). SO scattering reduces $\rho$ further (symplectic symmetry, exchange attraction $3 / 2$ ).

\section{N. Unitary Propagation}

We can put together the results discussed so far by generalizing eq. (98) to allow for complex (and matrix valued) parameters. In the originally encountered directed polymer (DP), the parameters $\nu>0$ and $\mu$ appearing in this equation were both real. To discuss the wavefunction in a magnetic field, we have to allow $\mu$ to take complex values. Finally, SO scattering is included by generalizing $W$ to a two component spinor, and using matrix valued $\mu$. We found that in all these cases the statistical behavior of $\ln W(\mathbf{x}, t)$ is the same. Is this a general property of eq. (98), independent of the choice of parameters? A special limit of this equation is when both $\mu \rightarrow-i \mu$ and $\nu \rightarrow-i \nu$ are purely imaginary. Then eq. (98) reduces to the Schrödinger equation

$$
i \frac{\partial W}{\partial t}=\left[\nu \nabla^{2}+\mu(\mathbf{x}, t)\right] W
$$

for a particle in a random time dependent potential. This equation has been considered in the context of particle diffusion in crystals at finite temperature [90 92], and to model the environment of a light test particle in a gas of much heavier particles [93]. Several authors 94 96] have also suggested that the diffusion of directed wave fronts in disordered media are described by eq. (137).

The path-integral solution to eq. (137) is [1,97.

$$
W(\mathbf{x}, t)=\int_{(\mathbf{0}, 0)}^{(\mathbf{x}, t)} \mathcal{D} \mathbf{x}\left(t^{\prime}\right) \exp \left\{-i \int_{0}^{t} d t^{\prime}\left[\frac{1}{4 \nu}\left(\frac{d \mathbf{x}}{d t^{\prime}}\right)^{2}+\mu\left(\mathbf{x}\left(t^{\prime}, t^{\prime}\right)\right]\right\},\right.
$$

where $\mathbf{x}\left(t^{\prime}\right)$ now describes a path in $d-1$ dimensions. In writing eq. (138), we have chosen the standard initial condition that at time $t=0$, the "wave function" is localized at the origin. The beam positions $\overline{\left\langle\mathbf{x}^{2}\right\rangle}$ and $\overline{\langle\mathbf{x}\rangle^{2}}$ characterize the transverse fluctuations of a directed beam $W$ about the forward path of least scattering. Here we use $\langle\cdots\rangle$ to indicate an average with the weight $|W(x, t)|^{2}$ for a given realization, and $\cdots$ to indicate quenched averaging over all realizations of randomness. Roughly speaking, $\overline{\langle\mathbf{x}\rangle^{2}}$ describes 
the wandering of the beam center, while $\overline{\left.\left\langle\mathbf{x}^{2}\right\rangle-<\mathbf{x}\right\rangle^{2}}$ provides a measure of the beam width.

A special property of eq. (137) which is valid only for real $\nu$ and $\mu$ is unitarity, i.e. the norm $\int d \mathbf{x}|W(\mathbf{x}, t)|^{2}$ is preserved at all times. (In the DP and tunnelling problems, the norm clearly decays with the length $t$.) This additional conservation law sets apart the random directed wave problem from DP, and in a sense makes its solution more tractable. This unitarity is of course a natural consequence of particle conservation for the Schrödinger equation, but has no counterpart for directed wave propagation. It is likely that a beam of light propagating in a random medium will suffer a loss of intensity, due to either back-reflection, inelastic scattering, or localization phenomena 98].

A number of efforts at understanding unitary propagation in random media have focused on the scaling behavior of the beam positions $\overline{\left\langle\mathbf{x}^{2}\right\rangle}$ and $\overline{\langle\mathbf{x}\rangle^{2}}$ at large $t$. Lattice models have been used here with some success. It has been shown using density-matrix techniques, for instance, that $\overline{\left\langle\mathrm{x}^{2}\right\rangle}$ scales linearly in time as a consequence of unitarity[91]; recent numerical simulations [99,100] also support this view. The scaling behavior of $\overline{\langle\mathbf{x}\rangle^{2}}$ at large $t$, however, is somewhat more complicated. An early numerical simulation in this area in ref. [96], employed a discretization procedure in which the norm of the wave function was not strictly preserved. In $2 d$, they found that $\overline{|\langle\mathbf{x}\rangle|}$ grew superdiffusively as $t^{\zeta}$ with $\zeta \approx 3 / 4$, while in $3 d$, they found a phase transition separating regimes of weak and strong disorder. Subsequent numerical studies [99 on directed waves in $2 d$ cast doubt on the validity of these results when the time evolution is strictly unitary, indicating that $\overline{\langle\mathbf{x}\rangle^{2}}$ scales subdiffusively in $2 d$ with $\zeta \approx 0.3$.

Somewhat surprising is the fact that a continuum formulation of the wave problem leads to different results. An exact treatment of the continuum Schrödinger equation(137) has been given by Jayannavar and Kumar [92]. They show that for a random potential $\delta$-correlated in time, $\overline{\left\langle\mathbf{x}^{2}\right\rangle} \sim t^{3}$ as $t \rightarrow \infty$. This behavior is modified when there are shortrange correlations in time 93, but the motion remains non-diffusive in that the particle is accelerated indefinitely as $t \rightarrow \infty$. Lattice models introduce a momentum cutoff $p_{\max } \sim$ $a^{-1}$, where $a$ is the lattice spacing, and therefore do not exhibit this effect. The momentum cutoff generated by the lattice discretization is in some sense artificial. Nevertheless, in a real fluctuating medium, we do expect on large time scales to recover the lattice result, i.e. normal diffusion. The reason is that dissipative effects do generate an effective momentum cutoff in most physical systems. (Strictly speaking, even in the absence of dissipation, relativistic constraints lead to a velocity cutoff $v=c$.) The presence of such a cutoff for 
the wave propagation problem, and hence the physical relevance of lattice versus continuum models, is still a matter of debate. While there is no underlying lattice, one suspects on physical grounds that there does exist an effective momentum cutoff for propagating waves, related to the speed of light in the background medium.

Previous numerical investigations of this problem start with a discretization of the parabolic wave equation in eq. (137). By contrast, we assume that the path integral representation is more fundamental and provide a direct discretization of eq. (138) that preserves unitarity [101]. For concreteness, we introduce the model in $2 d$. A discussion of its generalization to higher dimensions is taken up later. As usual, we identify the time axis with the primary direction of propagation and orient it along the diagonal of the square lattice. The wave function is defined on the bonds of this lattice. We use $W_{ \pm}(x, t)$ to refer to the amplitude for arriving at the site $(x, t)$ from the $\pm x$ direction. At $t=0$, the wave function is localized at the origin, with $W_{ \pm}(0,0)=1 / \sqrt{2}$. Transfer matrix techniques are now used to simulate diffusion in the presence of disorder. At time $t$, we imagine that a random scattering event occurs at each site on the lattice at which either $W_{+}(x, t)$ or $W_{-}(x, t)$ is non-zero. We implement these events by assigning to each scattering site a $2 \times 2$ unitary matrix $S(x, t)$. The values of the wave function at time $t+1$ are then computed from the recursion relation:

$$
\left(\begin{array}{l}
W_{-}(x+1, t+1) \\
W_{+}(x-1, t+1)
\end{array}\right)=\left(\begin{array}{ll}
S_{11}(x, t) & S_{12}(x, t) \\
S_{21}(x, t) & S_{22}(x, t)
\end{array}\right)\left(\begin{array}{l}
W_{-}(x, t) \\
W_{+}(x, t)
\end{array}\right)
$$

The $S$-matrices are required to be unitary in order to locally preserve the norm of the wave function. As a particular realization, we may consider the rotation matrix

$$
S(\theta, \phi)=\left(\begin{array}{cc}
\cos (\theta / 2) e^{i \phi} & \sin (\theta / 2) e^{-i \phi} \\
-\sin (\theta / 2) e^{i \phi} & \cos (\theta / 2) e^{-i \phi}
\end{array}\right)
$$

A physical realization of this model is obtained by placing semi polished mirrors of variable thickness, parallel to the $t$ axis, on the sites of a square lattice. Within this framework, it should be clear that the value of $W_{ \pm}(x, t)$ is obtained by summing the individual amplitudes of all directed paths which start at the origin and arrive at the point $(x, t)$ from the $\pm x$ direction. We thus have a unitary discretization of the path integral in eq. (138) in which the phase change from the potential $\mu(x, t)$ is replaced by an element of the matrix $S(x, t)$. A lattice $S$-matrix approach for the study of electron localization and the quantum Hall effect has been used by Chalker and Coddington [102]. A related model has also been recently proposed [103] to investigate the localization of wave packets in random media. These models also include back scattering and hence involve a larger matrix at each site. 


\section{O. Unitary Averages}

A particularly nice feature of unitary propagation is that the weights $W(x, t)$ are automatically normalized. In particular, we are interested in the beam positions

$$
\overline{<x^{2}(t)>}=\sum_{x} \overline{P(x, t)} x^{2}
$$

and

$$
\overline{<x(t)>^{2}}=\sum_{x_{1}, x_{2}} \overline{P\left(x_{1}, t\right) P\left(x_{2}, t\right)} x_{1} x_{2} \quad,
$$

where $P(x, t)$ is the probability distribution function on the lattice at time $t$, defined by

$$
P(x, t)=\left|W_{+}(x, t)\right|^{2}+\left|W_{-}(x, t)\right|^{2} \quad .
$$

(Defining the weights directly on the bonds does not substantially change the results.) Note that unlike the DP problem, $P(x, t)$ is properly normalized, i.e.

$$
\sum_{x} P(x, t)=1
$$

and eqs. (141) and (142) are not divided by normalizations such as $\sum_{x} P(x, t)$. This simplification is a consequence of unitarity and makes the directed wave problem tractable.

The average $\cdots$, in eqs. (141) and (142) is to be performed over a distribution of $S$-matrices that closely resembles the corresponding distribution for $\mu$ in the continuum problem. However, by analogy to the DP problem we expect any disorder to be relevant. Hence, to obtain the asymptotic scaling behavior, we consider the extreme limit of strong scattering in which each matrix $S(x, t)$ is an independently chosen random element of the group $U(2)$. With such a distribution we lose any pre-asymptotic behavior associated with weak scattering [93]. The results are expected to be valid over a range of length scales $a \ll x \ll \xi$, where $a$ is a distance over which the change of phase due to randomness is around $2 \pi$, and $\xi$ is the characteristic length for the decay of intensity and breakdown of unitarity. In the language of path integrals, the quantity $\overline{P(x, t)}$ represents the average over a conjugate pair of paths (from $W_{ \pm}$and $W_{ \pm}^{*}$ respectively.) As in the random sign problem, the paths must be exactly paired to make a non-zero contribution (since $\overline{S_{\alpha \beta}}=0$. In the strong disorder limit, each step along the paired paths contributes a factor of $1 / 2$. (It can be easily checked from eq. (140) that $\overline{\left|S_{\alpha \beta}\right|^{2}}=\overline{\cos ^{2}(\theta / 2)}=\overline{\sin ^{2}(\theta / 2)}=1 / 2$.) Thus, in this limit, the effect of an impurity at $(x, t)$ is to redistribute the incident probability 
flux $P(x, t)$ at random in the $+x$ and $-x$ directions. On average, the flux is scattered symmetrically so that the disorder-averaged probability describes the event space of a classical random walk, i.e.

$$
\overline{P(x, t)}=\frac{t !}{\left(\frac{t-x}{2}\right) !\left(\frac{t+x}{2}\right) !} .
$$

Substituting this into eq. (141), we find $\overline{\left\langle x^{2}(t)>\right.}=t$, in agreement with previous studies 91.

Consider now the position of the beam center $\overline{\langle x(t)\rangle^{2}}$, given by eq. (142). Unlike $\overline{P(x, t)}$, the correlation function $\overline{P\left(x_{1}, t\right) P\left(x_{2}, t\right)}$ does not have a simple form. It involves a sum over four paths, collapsed into two pairs by randomness averaging. The center of mass coordinate $R=\left(x_{1}+x_{2}\right) / 2$, performs a random walk with $\overline{R^{2}}=t / 2$. Let us define a new correlation function for the relative coordinate $r=x_{2}-x_{1}$, as

$$
W_{2}(r, t)=\sum_{R} \overline{P(R-r / 2, t) P(R+r / 2, t)}
$$

with the initial condition

$$
W_{2}(r, t=0)=\delta_{r, 0}
$$

The value of $W_{2}(r, t)$ is the disorder-averaged probability that two paired paths, evolved in the same realization of randomness, are separated by a distance $r$ at time $t$, and can be computed as a sum over all configurations that meet this criteria. Consider now the evolution of two such pairs from time $t$ to $t+1$. Clearly, at times when $r \neq 0$, the two pairs behave as independent random walks. On the other hand, when $r=0$, there is an increased probability that the paths move together as a result of participating in the same scattering event. An event in which the pairs stay together is enhanced (since $\overline{\left|S_{\alpha \beta}\right|^{4}}=$ $\overline{\cos ^{4}(\theta / 2)}=\overline{\sin ^{4}(\theta / 2)}=3 / 8$ ), while one in which the pairs separate is diminished (since $\left.\overline{\sin ^{2}(\theta / 2) \cos ^{2}(\theta / 2)}=1 / 8\right)$. These observations lead to the following recursion relation for the evolution of $W_{2}(r, t)$,

$W_{2}(r, t+1)=\left(\frac{1+\epsilon \delta_{r, 0}}{2}\right) W_{2}(r, t)+\left(\frac{1-\epsilon \delta_{r, 2}}{4}\right) W_{2}(r-2, t)+\left(\frac{1-\epsilon \delta_{r,-2}}{4}\right) W_{2}(r+2, t)$.

The parameter $\epsilon \geq 0$ measures the tendency of the paths to stick together on contact. (If the $S$-matrix is uniformly distributed over the group $U(2)$, then $\epsilon=1 / 4$.) Note that $\sum_{r} W_{2}(r)$ is preserved, as required by unitarity. 
Using eq. (147), we evolved $W_{2}(r, t)$ numerically for various values of $0<\epsilon<1$ up to $t \leq 15000$. The position of the beam center was then calculated from

$$
\overline{<x(t)>^{2}}=\sum_{R, r}\left(R^{2}-\frac{r^{2}}{4}\right) \overline{P(R-r / 2, t) P(R+r / 2, t)}=\frac{t}{2}-\frac{1}{4} \sum_{r} W_{2}(r, t) r^{2}
$$

The results suggest quite unambiguously that $\overline{\langle x(t)\rangle^{2}}$ scales as $t^{2 \zeta}$, with $\zeta=1 / 4$. We emphasize here the utility of the $S$-matrix model for directed waves in random media. Not only does our final algorithm compute averages over disorder in an exact way, but it requires substantially less time to do so than simulations which perform averages by statistical sampling as in DPRM. We have in fact confirmed our $2 d$ results with these slower methods on smaller lattices $(t<2000)$.

The model is easily extended to higher dimensions. The wave function takes its values on the bonds of a lattice in $d$ dimensions. Random $d \times d$ dimensional $S$-matrices are then used to simulate scattering events at the sites of the lattice. When the matrices $S(\mathbf{x}, t)$ are distributed uniformly over the group $U(d)$, the same considerations as before permit one to perform averages over disorder in an exact way. In addition, one obtains the general result for $d \geq 2$ that $\overline{\left\langle\mathbf{x}^{2}\right\rangle}$ scales linearly in time. The computation of $\overline{\langle\mathbf{x}\rangle^{2}}$ in $\left.d\right\rangle 2$, of course, requires significantly more computer resources. We have computed $\overline{<\mathbf{x}\rangle^{2}}$ on a $d=3$ body-centered cubic lattice, starting from the appropriate generalization of eq. (147). The results for $t<3000$, indicate that $\overline{\langle\mathbf{x}\rangle^{2}}$ scales logarithmically in time.

The above numerical results can be understood by appealing to some well-known properties of random walks. Consider a random walker on a $D=d-1$ dimensional hypercubic lattice. We suppose, as usual, that the walker starts out at the origin, and that at times $t=0,1,2, \cdots$, the walker has probability $0<p \leq 1 / 2 D$ to move one step in any lattice direction and probability $1-2 D p$ to pause for a rest. The mean time $t_{0}$ spent by the walker at the origin grows as 104

$$
t_{0} \sim\left\{\begin{array}{rl}
t^{\frac{1}{2}} & (D=1) \\
\ln t & (D=2) \\
\text { constant } & (D=3)
\end{array} .\right.
$$

The numerical results indicate a similar scaling for the wandering of the beam center $\overline{<\mathbf{x}>^{2}}$ in $d=D+1$ dimensions, for $d=2$ and $d=3$. We now show that this equivalence is not coincidental; moreover, it strongly suggests that $d_{u}=3$ is a critical dimension for directed 
waves in random media. To this end, let us consider a continuum version of eq. (147), which in general dimensions takes the form

$$
W_{2}(\mathbf{r}, t+1)=W_{2}(\mathbf{r}, t)+\nabla^{2}\left[W_{2}\left(1-\epsilon \delta^{D}(\mathbf{r})+\cdots\right)\right]
$$

The asymptotic solution for $\epsilon=0$ is just a gaussian packet of width $\overline{r^{2}}=2 t$. We can next perform a perturbative calculation in $\epsilon$. However, simple dimensional analysis shows the corrections scale as powers of $\epsilon / r^{D} \sim \epsilon t^{-D / 2}$, and thus

$$
\lim _{t \rightarrow \infty} W_{2}(\mathbf{r}, t)=\frac{1}{(4 \pi t)^{D / 2}} \exp \left(-\frac{r^{2}}{4 t}\right)\left[1+\mathcal{O}\left(\epsilon t^{-(d-1) / 2}\right)\right] .
$$

Applying the above results to the continuum version of eq. (147), gives

$$
\begin{aligned}
\overline{\langle x\rangle_{t+1}^{2}}-\overline{\langle x\rangle_{t}^{2}} & =\frac{1}{2}-\frac{1}{4} \sum_{r}\left[W_{2}(r, t+1)-W_{2}(r, t)\right] r^{2} \\
& \simeq \frac{1}{2}-\frac{1}{4} \int d^{D} \mathbf{r} \nabla^{2}\left[W_{2}\left(1-\epsilon \delta^{D}(\mathbf{r})+\cdots\right)\right] \\
& \simeq \frac{1}{2}-\frac{1}{2} \int d^{D} \mathbf{r} W_{2}\left(1-\epsilon \delta^{D}(\mathbf{r})\right)=\epsilon W_{2}(0, t)
\end{aligned}
$$

Summing both sides of this equation over $t$, one finds

$$
\overline{<x(t+1)\rangle^{2}}=\epsilon \sum_{t^{\prime}=0}^{t} W_{2}\left(0, t^{\prime}\right) \approx \int_{0}^{t} d t^{\prime}\left(4 \pi t^{\prime}\right)^{-D / 2}
$$

The final integral is proportional to the time a random walker spends at the origin, and reproduces the results in eq. (149).

We can also regard $W_{2}(r, t)$ as a probability distribution function for the relative coordinate between two interacting random walkers. In this interpretation, the value of $\epsilon$ in eq. (147) parametrizes the strength of a contact interaction between the walkers. If $\epsilon=0$, the walkers do not interact at all; if $\epsilon=1$, the walkers bind on contact. According to eq. (153), the wandering of the beam center $\overline{\langle x(t)\rangle^{2}}$ is proportional to the mean number of times that the paths of these walkers intersect during time $t$. If $\epsilon=0$, the number of intersections during time $t$ obeys the scaling law in eq. (149), since in this case, the relative coordinate between the walkers performs a simple random walk. Numerical results indicate that the same scaling law applies when $0<\epsilon<1$ : the contact attraction does not affect the asymptotic properties of the random walk. In summary, three classes of behavior are possible in this model. For $\epsilon=0$, i.e. no randomness, the incoming beam stays centered 
at the origin, while its width grows diffusively. For $0<\epsilon<1$, the beam center, $\overline{<\mathbf{x}>^{2}}$, also fluctuates, but with a dimension dependent behavior as in eq. (149). In the limit of $\epsilon=1$, interference phenomena disappear completely. In this case, the beam width is zero and the beam center performs a simple random walk.

We conclude by comparing the situation here to that of DPRM. In the replica approach to DPRM, the $n$-th moment of the weight $W(x, t)$ is obtained from the statistics of $n$ directed paths. Disorder-averaging produces an attractive interaction between these paths with the result that they may form a bound state. In $d \leq 2$, any amount of randomness (and hence attraction) leads to the formation of a bound state. The behavior of the bound state energy can then be used to extract an exponent of $\zeta=2 / 3$ for superdiffusive wandering. By contrast, the replicated paths encountered in the directed wave problem (such as the two paths considered for eq. (145)), although interacting, cannot form a bound state [99], as it is inconsistent with unitarity. This result also emerges in a natural way from our model of directed waves. In $d=2$, for instance, it is easy to check that $W_{2}(\mathbf{r}) \sim\left(1-\epsilon \delta_{r, 0}\right)^{-1}$ is the eigenstate of largest eigenvalue for the evolution of the relative coordinate. Hence, as $t \rightarrow \infty$, for randomness $\delta$-correlated in space and time, there is no bound state. This result holds in $d \geq 2$ and is not modified by short-range correlations in the randomness. The probability-conserving nature of eq. (147) is crucial in this regard 105 as it precludes a $u \delta^{D}(\mathbf{r})$ attraction in eq. (150). Small perturbations that violate the conservation of probability lead to the formation of a bound state. In the language of the renormalization group, the scaling of directed waves in random media is governed by a fixed point that is unstable with respect to changes that do not preserve a strictly unitary evolution.

Subsequently, a number of authors have obtained additional results from the random $S$-matrix model. Following our work, Friedberg and Yu 106 calculated the leading terms in the scaling laws for the beam center in $d \geq 2$, and also the next-order corrections. The analytical results are in agreement with those presented above. Cule and Shapir [107] extended the methods of this section to compute the higher moments of the probability distribution for directed waves in random media. If this probability distribution is multifractal, as claimed in ref. [100], the higher moments should obey new scaling laws whose exponents are not simply related to those of the lower moments. Within the framework of the $S$-matrix model, Cule and Shapir did not find evidence for multifractal scaling, while suggesting that certain aspects of the scaling behavior may be sensitive to details of the unitary time evolution. 


\section{P. Summing all Paths in High Dimensions}

In the next few sections we shall return to the non-random Ising model. The high temperature series can be approximately summed so as to reproduce mean-field (gaussian) behavior. This correspondence provides a better understanding of why such behavior is applicable in high dimensions, and also prepares the way for the exact summation of the series in two dimensions (next section). We shall then use these methods to look at two dimensional random Ising models. The high temperature series for the partition function of the non-random Ising model on a $d$-dimensional hypercubic lattice is

$$
Z=\sum_{\left\{\sigma_{i}\right\}} e^{K \sum_{\langle i j\rangle} \sigma_{i} \sigma_{j}}=2^{N} \cosh ^{d N} K \times S,
$$

where $S$ is the sum over all allowed graphs on the lattice, each weighted by $\tau$ raised to the number of bonds in the graph, with $\tau \equiv \tanh K$. The allowed graphs have an even number of bonds per site. The simplest graphs have the topology of a single closed loop. There are also graphs composed of disconnected closed loops. Keeping in mind cumulant expansions, we set

$$
\Xi=\text { sum over contribution of all graphs with one loop , }
$$

and introduce another sum,

$$
\begin{aligned}
S^{\prime}=\exp (\Xi) & =1+\Xi+\frac{1}{2}(\Xi)^{2}+\frac{1}{6}(\Xi)^{3}+\cdots \\
& =1+(1 \text { loop graphs })+(2 \text { loop graphs })+(3 \text { loop graphs })+\cdots
\end{aligned}
$$

Despite their similarities, the sums $S$ and $S^{\prime}$ are not identical in that $S^{\prime}$ includes additional graphs where a particular bond contributes more than once. (In the original sum $S$, each lattice bond contributes a factor of 1 or $\tau$. After raising $\Xi$ to a power $n$, a particular bond may contribute up to $\tau^{n}$.) In a similar approximation, we shall allow additional closed paths in $\Xi$ in which a particular bond is traversed more than once in completing the loop. Qualitatively, $S$ is the partition function of a gas of self-avoiding polymer loops with a monomer fugacity of $\tau$. The self-avoiding constraint is left out in the partition function $S^{\prime}$, which thus corresponds to a gas of phantom polymer loops. The corresponding free energy is

$$
\begin{aligned}
\ln S^{\prime} & =\text { sum over all closed random walks on the lattice } \times \tau^{\text {length of walk }} \\
& =N \sum_{\ell} \frac{\tau^{\ell}}{\ell} \text { (number of closed walks of } \ell \text { steps starting and ending at } \mathbf{0} \text { ), }
\end{aligned}
$$


where extensivity is guaranteed since (up to boundary effects) the same loop can be started from any point on the lattice. The additional factor of $1 / \ell$ accounts for the $\ell$ possible starting points for a loop of length $\ell$. To count the number of paths we introduce a set of $N \times N$ matrices,

$$
\langle\mathbf{i}|W(\ell)| \mathbf{j}\rangle \equiv \text { number of walks from } \mathbf{j} \text { to } \mathbf{i} \text { in } \ell \text { steps }
$$

Then

$$
\frac{\ln S^{\prime}}{N}=\sum_{\ell} \frac{\tau^{\ell}}{\ell}\langle\mathbf{0}|W(\ell)| \mathbf{0}\rangle
$$

Similarly, the spin-spin correlation function

$$
\langle\sigma(\mathbf{0}) \sigma(\mathbf{r})\rangle=\frac{1}{Z} \sum_{\left\{\sigma_{i}\right\}} \sigma(\mathbf{0}) \sigma(\mathbf{r}) \prod_{\langle i j\rangle}\left(1+\tau \sigma_{i} \sigma_{j}\right)
$$

is related to the sum over all paths connecting the points $\mathbf{0}$ and $\mathbf{r}$ on the lattice. In addition to the simple paths that directly connect the two points, there are disconnected graphs that contain additional closed loops. In the same approximation of allowing all intersections between paths, the partition function $S^{\prime}$ can be factored out of the numerator and denominator of eq. (160), and

$$
\left\langle\sigma(\mathbf{0}) \sigma(\mathbf{r}\rangle \approx \sum_{\ell} \tau^{\ell}\langle\mathbf{r}|W(\ell)| \mathbf{0}\rangle\right.
$$

As the walks are Markovian, their number can be calculated recursively. First note that any walk from $\mathbf{0}$ to $\mathbf{r}$ in $\ell$ steps can be regarded as a walk from $\mathbf{0}$ to some other point $\mathbf{r}^{\prime}$ in $\ell-1$ steps, followed by a single step from $\mathbf{r}^{\prime}$ to $\mathbf{r}$. Summing over all possible locations of the intermediate point leads to

$$
\begin{aligned}
\langle\mathbf{r}|W(\ell)| \mathbf{0}\rangle & =\sum_{\mathbf{r}^{\prime}}\left\langle\mathbf{r}|W(1)| \mathbf{r}^{\prime}\right\rangle \times\left\langle\mathbf{r}^{\prime}|W(\ell-1)| \mathbf{0}\right\rangle \\
& =\langle\mathbf{r}|T W(\ell-1)| \mathbf{0}\rangle \quad,
\end{aligned}
$$

where the sum corresponds to the product of two matrices and we have defined $T \equiv W(1)$. The recursion process can be continued and

$$
W(\ell)=T W(\ell-1)=T^{2} W(\ell-2)^{2}=\cdots=T^{\ell}
$$

Thus all lattice random walks are generated by the transfer matrix $T$, whose elements are

$$
\left\langle\mathbf{r}|T| \mathbf{r}^{\prime}\right\rangle=\left\{\begin{array}{ll}
1 & \text { if } \mathbf{r} \text { and } \mathbf{r}^{\prime} \text { are nearest neighbors } \\
0 & \text { otherwise }
\end{array} .\right.
$$


For example in $d=2$,

$$
\left\langle x, y|T| x^{\prime}, y^{\prime}\right\rangle=\delta_{y, y^{\prime}}\left(\delta_{x, x^{\prime}+1}+\delta_{x, x^{\prime}-1}\right)+\delta_{x, x^{\prime}}\left(\delta_{y, y^{\prime}+1}+\delta_{y, y^{\prime}-1}\right)
$$

and successive actions of $T$ on a walker starting at the origin, $\mid x, y>=\delta_{x, 0} \delta_{y, 0}$, generate the patterns

$$
\begin{array}{llllll}
0 & 0 & 0 \\
0 & 1 & 0 \\
0 & 0 & 0
\end{array} \longrightarrow \begin{array}{lll}
0 & 1 & 0 \\
1 & 0 & 1 \\
0 & 1 & 0
\end{array} \longrightarrow \begin{array}{lllll}
0 & 0 & 1 & 0 & 0 \\
0 & 2 & 0 & 2 & 0 \\
1 & 0 & 4 & 0 & 1 \\
0 & 2 & 0 & 2 & 0 \\
0 & 0 & 1 & 0 & 0
\end{array} \longrightarrow \cdots
$$

The value at each site is the number of walks ending at that point after $\ell$ steps.

Various properties of random walks can be deduced from diagonalizing the matrix $T$. Due to the translational symmetry of the lattice, this is achieved in the Fourier basis $\langle\mathbf{r} \mid \mathbf{q}\rangle=e^{i \mathbf{q} \cdot \mathbf{r}} / \sqrt{N}$. For example, in $d=2$ starting from eq(165), it can be checked that

$$
\begin{aligned}
\left\langle x, y|T| q_{x}, q_{y}\right\rangle & =\sum_{x^{\prime}, y^{\prime}}\left\langle x, y|T| x^{\prime}, y^{\prime}\right\rangle\left\langle x^{\prime}, y^{\prime} \mid q_{x}, q_{y}\right\rangle \\
& =\frac{1}{\sqrt{N}}\left[e^{i q_{y} y}\left(e^{i q_{x}(x+1)}+e^{i q_{x}(x-1)}\right)+e^{i q_{x} x}\left(e^{i q_{y}(y+1)}+e^{i q_{y}(y-1)}\right)\right] \\
& =\frac{1}{\sqrt{N}} e^{i\left(q_{x} x+q_{y} y\right)}\left[2 \cos q_{x}+2 \cos q_{y}\right]=T\left(q_{x}, q_{y}\right)\left\langle x, y \mid q_{x}, q_{y}\right\rangle
\end{aligned}
$$

The generalized eigenvalue for a $d$-dimensional hypercubic lattice is

$$
T(\mathbf{q})=2 \sum_{\alpha=1}^{d} \cos q_{\alpha} .
$$

The correlation function in eq. (161) is now evaluated as

$$
\begin{aligned}
\langle\sigma(\mathbf{r}) \sigma(\mathbf{0})\rangle & \approx \sum_{\ell}^{\infty} \tau^{\ell}\langle\mathbf{r}|W(\ell)| \mathbf{0}\rangle=\sum_{\ell}^{\infty}\left\langle\mathbf{r}\left|(\tau T)^{\ell}\right| \mathbf{0}\right\rangle \\
= & \left\langle\mathbf{r}\left|\frac{1}{1-\tau T}\right| \mathbf{0}\right\rangle=\sum_{\mathbf{q}}\langle\mathbf{r} \mid \mathbf{q}\rangle \frac{1}{1-\tau T(\mathbf{q})}\langle\mathbf{q} \mid \mathbf{0}\rangle \\
= & N \int \frac{d^{d} \mathbf{q}}{(2 \pi)^{d}} \frac{e^{i \mathbf{q} \cdot \mathbf{r}}}{N} \frac{1}{1-2 \tau \sum_{\alpha=1}^{d} \cos q_{\alpha}}=\int \frac{d^{d} \mathbf{q}}{(2 \pi)^{d}} \frac{e^{i \mathbf{q} \cdot \mathbf{r}}}{1-2 \tau \sum_{\alpha} \cos q_{\alpha}} .
\end{aligned}
$$

For $\tau \rightarrow 0$, the shortest path costs least energy and $\langle\sigma(\mathbf{0}) \sigma(\mathbf{r})\rangle \sim \tau^{|\mathbf{r}|}$. As $\tau$ increases, larger paths dominate the sum because they are more numerous (i.e. entropically favored). Eventually there is a singularity for $1-\tau T(\mathbf{0})=0$, i.e. at $2 d \times \tau_{c}=1$, when arbitrarily 
long paths become important. For $\tau<\tau_{c}$, the partition function is dominated by small loops, and a polymer connecting two far away points is stretched by its line tension. When the fugacity exceeds $\tau_{c}$, the line tension vanishes and loops of arbitrary size are generated. Clearly the neglect of intersections (which leads to a finite density) is no longer justified in this limit. This transition is the manifestation of Ising ordering in the language of paths representing the high temperature series. On approaching the transition from the high temperature side, the sums are dominated by very long paths. Accordingly, the denominator of eq. (168) can be expanded for small $\mathbf{q}$ as

$$
1-\tau T(\mathbf{q})=1-2 \tau \sum_{\alpha=1}^{d} \cos q_{\alpha} \approx(1-2 d \tau)+\tau q^{2}+\mathcal{O}\left(q^{4}\right) \approx \tau_{c}\left(\xi^{-2}+q^{2}+\mathcal{O}\left(q^{4}\right)\right),
$$

where

$$
\xi \equiv\left(\frac{1-2 d \tau}{\tau_{c}}\right)^{-1 / 2}
$$

The resulting correlation functions, $\langle\sigma(\mathbf{0}) \sigma(\mathbf{r})\rangle \propto \int \frac{d^{d} \mathbf{q}}{(2 \pi)^{d}} e^{i \mathbf{q} \cdot \mathbf{r}} /\left(q^{2}+\xi^{-2}\right)$, are identical to those obtained from a free (gaussian) field theory, and

$$
\langle\sigma(\mathbf{0}) \sigma(\mathbf{r})\rangle \propto\left\{\begin{array}{rr}
\frac{1}{r^{d-2}} & \text { for } r \ll \xi \\
\frac{e^{-r / \xi}}{r^{(d-1) / 2}} & \text { for } r \gg \xi
\end{array} .\right.
$$

The correlation length in eq. (170) diverges as $\xi \sim\left(\tau_{c}-t\right)^{-\nu}$, with the exponent $\nu=1 / 2$.

We can also calculate the free energy in eq. (159) as

$$
\begin{aligned}
\frac{\ln S^{\prime}}{N} & =\sum_{\ell}^{\infty} \frac{\tau^{\ell}}{\ell}\langle\mathbf{0}|W(\ell)| \mathbf{0}\rangle=\left\langle\mathbf{0}\left|\sum_{\ell}^{\infty} \frac{\tau^{\ell} T^{\ell}}{\ell}\right| \mathbf{0}\right\rangle \\
& =-\langle\mathbf{0}|\ln (1-\tau T)| \mathbf{0}\rangle=-N \int \frac{d^{d} \mathbf{q}}{(2 \pi)^{d}}\langle\mathbf{0} \mid \mathbf{q}\rangle \ln (1-\tau T(\mathbf{q}))\langle\mathbf{q} \mid \mathbf{0}\rangle \\
& =-\int \frac{d^{d} \mathbf{q}}{(2 \pi)^{d}} \ln \left(1-2 \tau \sum_{\alpha=1}^{d} \cos q_{\alpha}\right) .
\end{aligned}
$$

In the vicinity of the critical point at $\tau_{c}=1 /(2 d)$ the argument of the logarithm is proportional to $\left(q^{2}+\xi^{-2}\right)$ from eq. (169). This is precisely the free energy of a free field theory, and scales as

$$
f_{\text {sing }} \propto \xi^{-d} \propto\left(\tau_{c}-\tau\right)^{d / 2} .
$$


The singular part of the heat capacity, obtained after taking two derivatives, is governed by the exponent $\alpha=2-d / 2$. Note that in evaluating the sums appearing in eqs. (168) and (172) the lower limit for $\ell$ is not treated carefully. The series in eq. (168) is assumed to start from $\ell=0$, and that of eq. (172) from $\ell=1$. In fact the first few terms of both series may be zero because the number of steps is not sufficient to reach $\mathbf{0}$ from $\mathbf{r}$, or to from a closed loop. This is not a serious omission in that the singular behavior of a series is not effected by its first few terms. Treating the first few terms properly can only add analytic corrections to the singular forms calculated in eqs. (168) and (172).

The equivalence of these results to a free field theory is a manifestation of field-particle duality. In a field theoretical description, (imaginary) time appears as an additional dimension, and the two point correlations describe the probability of propagating a particle from one point in space-time to another. In a wave description, this probability is calculated by evolving the wave function using the Schrödinger equation. Alternatively, the probability can be calculated as the sum over all (Feynman) paths connecting the two points, each path weighted with the correct action. The second sum is similar to the above calculation of $\langle\sigma(\mathbf{r}) \sigma(\mathbf{0})\rangle$.

This approach provides an interesting geometrical interpretation of the phase transition. The establishment of long range order implies that all parts of the system have selected the same state. This information is carried by the bonds connecting nearest neighbors, and can be passed from the origin to a point $\mathbf{r}$ through all paths connecting these two points. The fugacity $\tau$ is a measure of the reliability of information transfer between neighboring sites. Along a one dimensional chain, unless $\tau=1$, the transferred information decays at large distances and it is impossible to establish long range order. In higher dimensions there are many more paths, and by accumulating the information from all paths, it is possible to establish order at $\tau_{c}<1$. Since the number of paths of length $\ell$ grows as $(2 d)^{\ell}$ while their information content decays as $\tau^{\ell}$, the transition occurs at $\tau_{c}=1 /(2 d)$. (A better approximation is obtained by including some of the constraints by noting that the random walk cannot back track. In this case the number of walks grows as $(2 d-1)^{\ell}$.) The total information from paths of length $\ell$ is weighted by $(2 d \tau)^{\ell}$, and decays exponentially for $\tau<\tau_{c}$. The characteristic path length, $\bar{\ell}=-1 / \ln (2 d \tau)$, diverges as $\left(\tau_{c}-\tau\right)^{-1}$ on approaching the transition. For paths of size $\ell \ll \bar{\ell}$ there is very good information transfer. Such paths execute random walks on the lattice and cover a distance $\xi \approx \bar{\ell}^{1 / 2}$. The divergence of $\nu$ with an exponent of $1 / 2$ is thus a consequence of the random walk nature of the paths. 
Why does the classical picture fail for $d \leq 4$ ? Let us focus on the dominant paths close to the phase transition. Is it justified to ignore the intersections of such paths? Random walks can be regarded as geometrical entities of fractal (Hausdorf) dimension $d_{f}=2$. This follows from the general definition of dimension relating the mass and extent of an object by $M \propto R^{d_{f}}$. The size of a random walk $(R \propto \xi)$ is the square root of its length $(M \propto \ell)$. Two geometrical entities of dimensions $d_{1}$ and $d_{2}$ will generally intersect in $d$-dimensional space if $d_{1}+d_{2} \geq d$. Thus our random walkers are unlikely to intersect in $d \geq d_{u}=2+2=4$, and the results obtained by neglecting intersections are asymptotically valid. Below the upper critical dimension of 4 , random walks have frequent encounters and their intersections must be treated correctly. The diagrams obtained in the perturbative calculation of the propagator in a $\phi^{4}$ theory correspond precisely to taking into account the intersections of paths. (Each vertex corresponds to one intersection.) It is now clear that the constraint of self-avoidance will swell the paths beyond their random walk size leading to an increase in the exponent $\nu$. Below the transition, the length of dominant paths grows without bound and the self-avoiding constraint is always necessary.

\section{Q. The Ising Model on a Square Lattice}

As indicated in eq. (154), the Ising partition function is related to a sum $S$, over collections of paths on the lattice. The allowed graphs for a square lattice have 2 or 4 bonds per site. Each bond can appear only once in each graph, contributing a factor of $\tau \equiv \tanh K$. While it is tempting to replace $S$ with the exactly calculable sum $S^{\prime}$, of all loops of random walks on the lattice, this leads to an overestimation of $S$. The differences between the two sums arise from intersections of random walks and can be divided into two categories:

(a) There is an over-counting of graphs which intersect at a site, i.e. with 4 bonds through a point. Consider a graph composed of two loops meeting at a site. Since a walker entering the intersection has three choices, this graph can be represented by three distinct random walks. One choice leads to two disconnected loops; the other two are single loops with or without a self-crossing in the walker's path.

(b) The independent random walkers in $S^{\prime}$ may go through a particular lattice bond more than once.

Including these constraints amounts to introducing interactions between paths. The resulting interacting random walkers are non-Markovian, as each step is no longer independent of previous ones and of other walkers. While such interacting walks are not in 
general amenable to exact treatment, in two dimensions an interesting topological property allows us to make the following assertion:

$$
\begin{gathered}
S=\sum \text { collections of loops of random walks with no U turns } \\
\times \tau^{\text {number of bonds }} \times(-1)^{\text {number of crossings }} .
\end{gathered}
$$

The negative signs for some terms reduce the overestimate and render the exact sum.

Proof: We shall deal in turn with the two problems mentioned above

(a) Consider a graph with many intersections and focus on a particular one. A walker must enter and leave such an intersection twice. This can be done in three ways, only one of which involves the path of the walker crossing itself (when the walker proceeds straight through the intersection). This configuration carries an additional factor of (-1) according to eq. (174). Thus, independent of other crossings, these three configurations sum up to contribute a factor of 1 . By repeating this reasoning at each intersection we see that the over-counting problem is removed, and the sum over all possible ways of tracing the graph leads to the correct factor of one.

(b) Consider a bond that is crossed by two walkers (or twice by the same walker). We can imagine the bond as an avenue with two sides. For each configuration in which the two paths enter and leave on the same side of the avenue, there is another one in which the paths go to the opposite side. The latter involves a crossing of paths and hence carries a minus sign with respect to the former. The two possibilities thus cancel out! The reasoning can be generalized to multiple passes through any bond. The only exception is when the doubled bond is created as a result of a U-turn. This is why such backward steps are explicitly excluded from eq. (174).

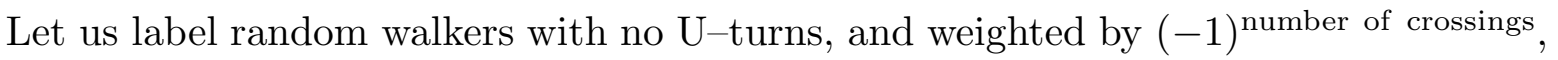
as $\mathrm{RW}^{*}$ s. Then as in eq. (156) the terms in $S$ can be organized as

$$
\begin{aligned}
S & =\sum\left(\mathrm{RW}^{*} \mathrm{~s} \text { with } 1 \text { loop }\right)+\sum\left(\mathrm{RW}^{*} \mathrm{~s} \text { with } 2 \text { loops }\right)+\sum\left(\mathrm{RW}^{*} \text { s with } 3 \text { loops }\right)+\cdots \\
& =\exp \left[\sum\left(\mathrm{RW}^{*} \text { s with } 1 \text { loop }\right)\right] .
\end{aligned}
$$

The exponentiation of the sum is justified since the only interaction between $\mathrm{RW}^{*} \mathrm{~s}$ is the sign related to their crossings. As two RW* loops always cross an even number of times, this is equivalent to no interaction at all. Using eq. (154), the full Ising free energy is calculated as

$$
\ln Z=N \ln 2+2 N \ln \cosh K+\sum\left(\mathrm{RW}^{*} \mathrm{~s} \text { with } 1 \text { loop } \times \tau^{\# \text { of bonds }}\right)
$$


Organizing the sum in terms of the number of bonds, and taking advantage of the translational symmetry of the lattice (up to corrections due to boundaries),

$$
\frac{\ln Z}{N}=\ln \left(2 \cosh ^{2} K\right)+\sum_{\ell}^{\infty} \frac{\tau^{\ell}}{\ell}\left\langle\mathbf{0}\left|W^{*}(\ell)\right| \mathbf{0}\right\rangle
$$

where

$$
\begin{aligned}
\left\langle\mathbf{0}\left|W^{*}(\ell)\right| \mathbf{0}\right\rangle= & \text { number of closed loops of } \ell \text { steps, with no } U \text { turns, from } \mathbf{0} \text { to } \mathbf{0} \\
& \times(-1)^{\# \text { of crossings }} .
\end{aligned}
$$

The absence of U-turns, a local constraint, does not complicate the counting of walks. On the other hand, the number of crossings depends on all sites previously crossed by the walker and is a non-Markovian property. Fortunately, in two dimensions it is possible to obtain the parity of the number of crossings from local considerations. The first step is to make the walks oriented by placing an arrow along the direction that the path is traversed. Since any path can be traversed in two directions,

$$
\left\langle\mathbf{0}\left|W^{*}(\ell)\right| \mathbf{0}\right\rangle=\frac{1}{2} \sum \text { oriented } \mathrm{RW}^{*} \text { loops of } \ell \text { steps, no } \mathrm{U} \text { turns, from } \mathbf{0} \text { to } \mathbf{0} \times(-1)^{n_{c}},
$$

where $n_{c}$ is the number of self-crossings of the loop. We can now take advantage of the following topological result 108:

Whitney's Theorem: The number of self-crossings of a planar loop is related to the total angle $\Theta$, through which the tangent vector turns in going around the loop by

$$
\left(n_{c}\right)_{\bmod 2}=\left(1+\frac{\Theta}{2 \pi}\right)_{\bmod 2} .
$$

This theorem can be checked by a few examples. A single loop corresponds to $\Theta= \pm 2 \pi$, while a single intersection results in $\Theta=0$.

Since the total angle $\Theta$ is the sum of the angles through which the walker turns at each step, the parity of crossings can be obtained using local information alone, as

$$
(-1)^{n_{c}}=e^{i \pi n_{c}}=\exp \left[i \pi\left(1+\frac{\Theta}{2 \pi}\right)\right]=-e^{\frac{i}{2} \sum_{j=1}^{\ell} \theta_{j}},
$$

where $\theta_{j}$ is the angle through which the walker turns on the $j^{\text {th }}$ step. Hence

$$
\begin{aligned}
\left\langle\mathbf{0}\left|W^{*}(\ell)\right| \mathbf{0}\right\rangle= & -\frac{1}{2} \sum \text { oriented } \mathrm{RW}^{*} \text { loops of } \ell \text { steps, with no } \mathrm{U} \text { turns, from } \mathbf{0} \text { to } \mathbf{0} \\
& \times \exp \left(\frac{1}{2} \sum \text { local change of angle by the tangent vector }\right)
\end{aligned}
$$


The angle turned can be calculated at each site if we keep track of the directions of arrival and departure of the path. To this end, we introduce a label $\mu$ for the 4 directions going out of each site, e.g. $\mu=1$ for right, $\mu=2$ for up, $\mu=3$ for left, and $\mu=4$ for down. We next introduce a set of $4 N \times 4 N$ matrices generalizing eq. (158) to

$\left\langle x_{2} y_{2}, \mu_{2}\left|W^{*}(\ell)\right| x_{1} y_{1}, \mu_{1}\right\rangle=\sum$ oriented random walks of $\ell$ steps, with no $\mathrm{U}$ turns, departing $\left(x_{1}, y_{1}\right)$ along $\mu_{1}$, proceeding along $\mu_{2}$ after reaching $\left(x_{2}, y_{2}\right) \times e^{\frac{i}{2} \sum_{j=1}^{\ell} \theta_{j}}$

Thus $\mu_{2}$ specifies a direction taken after the walker reaches its destination. It serves to exclude some paths (e.g., arriving along $-\mu_{2}$ ), and leads to an additional phase. As in eq. (162), due to their Markovian property, these matrices can be calculated recursively as

$$
\begin{aligned}
& \left\langle x_{2} y_{2}, \mu_{2}\left|W^{*}(\ell)\right| x_{1} y_{1}, \mu_{1}\right\rangle= \\
& \sum_{x^{\prime} y^{\prime}, \mu^{\prime}}\left\langle x_{2} y_{2}, \mu_{2}\left|T^{*}\right| x^{\prime} y^{\prime}, \mu^{\prime}\right\rangle\left\langle x^{\prime} y^{\prime}, \mu^{\prime}\left|W^{*}(\ell-1)\right| x_{1} y_{1}, \mu_{1}\right\rangle= \\
& \left\langle x_{2} y_{2}, \mu_{2}\left|T^{*} W^{*}(\ell-1)\right| x_{1} y_{1}, \mu_{1}\right\rangle=\left\langle x_{2} y_{2}, \mu_{2}\left|T^{* \ell}\right| x_{1} y_{1}, \mu_{1}\right\rangle,
\end{aligned}
$$

where $T^{*} \equiv W^{*}(1)$ describes one step of the walk. The direction of arrival uniquely determines the nearest neighbor from which the walker departed, and the angle between the two directions fixes the phase of the matrix element. We thus generalize eq. (165) to a $4 \times 4$ matrix that keeps track of both connectivity and phase between pairs of sites, i.e.

$$
\begin{aligned}
& \left\langle x^{\prime} y^{\prime}\left|T^{*}\right| x y\right\rangle= \\
& {\left[\begin{array}{cccc}
\left\langle x^{\prime} y^{\prime} \mid x+1 y\right\rangle & \left\langle x^{\prime} y^{\prime} \mid x+1 y\right\rangle e^{\frac{i \pi}{4}} & 0 & \left\langle x^{\prime} y^{\prime} \mid x+1 y\right\rangle e^{-\frac{i \pi}{4}} \\
\left\langle x^{\prime} y^{\prime} \mid x y+1\right\rangle e^{-\frac{i \pi}{4}} & \left\langle x^{\prime} y^{\prime} \mid x y+1\right\rangle & \left\langle x^{\prime} y^{\prime} \mid x y+1\right\rangle e^{\frac{i \pi}{4}} & 0 \\
0 & \left\langle x^{\prime} y^{\prime} \mid x-1 y\right\rangle e^{-\frac{i \pi}{4}} & \left\langle x^{\prime} y^{\prime} \mid x-1 y\right\rangle & \left\langle x^{\prime} y^{\prime} \mid x-1 y\right\rangle e^{\frac{i \pi}{4}} \\
\left\langle x^{\prime} y^{\prime} \mid x y-1\right\rangle e^{\frac{i \pi}{4}} & 0 & \left\langle x^{\prime} y^{\prime} \mid x y-1\right\rangle e^{-\frac{i \pi}{4}} & \left\langle x^{\prime} y^{\prime} \mid x y-1\right\rangle
\end{array}\right],}
\end{aligned}
$$

where $<x y \mid x^{\prime} y^{\prime}>\equiv \delta_{x, x^{\prime}} \delta_{y, y^{\prime}}$

Because of its translational symmetry, the $4 N \times 4 N$ matrix takes a block diagonal form in the Fourier basis $\left\langle x y \mid q_{x} q_{y}\right\rangle=e^{i\left(q_{x} x+q_{y} y\right)} / \sqrt{N}$, i.e.

$$
\sum_{x y}\left\langle x^{\prime} y^{\prime}, \mu^{\prime}\left|T^{*}\right| x y, \mu\right\rangle\left\langle x y \mid q_{x} q_{y}\right\rangle=\left\langle\mu^{\prime}\left|T^{*}(\mathbf{q})\right| \mu\right\rangle\left\langle x^{\prime} y^{\prime} \mid q_{x} q_{y}\right\rangle
$$

Each $4 \times 4$ block is labelled by a wavevector $\mathbf{q}=\left(q_{x}, q_{y}\right)$, and takes the form

$$
T^{*}(\mathbf{q})=\left[\begin{array}{cccc}
e^{-i q_{x}} & e^{-i\left(q_{x}-\frac{\pi}{4}\right)} & 0 & e^{-i\left(q_{x}+\frac{\pi}{4}\right)} \\
e^{-i\left(q_{y}+\frac{\pi}{4}\right)} & e^{-i q_{y}} & e^{-i\left(q_{y}-\frac{\pi}{4}\right)} & 0 \\
0 & e^{i\left(q_{x}-\frac{\pi}{4}\right)} & e^{i q_{x}} & e^{i\left(q_{x}+\frac{\pi}{4}\right)} \\
e^{i\left(q_{y}+\frac{\pi}{4}\right)} & 0 & e^{i\left(q_{y}-\frac{\pi}{4}\right)} & e^{i q_{y}}
\end{array}\right] .
$$


To ensure that a path that starts at the origin completes a loop properly, the final arrival direction at the origin must coincide with the original one. Summing over all 4 such directions, the total number of such loops is obtained from

$$
\left\langle\mathbf{0}\left|W^{*}(\ell)\right| \mathbf{0}\right\rangle=\sum_{\mu=1}^{4}\left\langle 00, \mu\left|T^{* \ell}\right| 00, \mu\right\rangle=\frac{1}{N} \sum_{x y, \mu}\left\langle x y, \mu\left|T^{* \ell}\right| x y, \mu\right\rangle=\frac{1}{N} \operatorname{tr}\left(T^{* \ell}\right)
$$

Using eq. (177), the free energy is calculated as

$$
\begin{aligned}
\frac{\ln Z}{N} & =\ln \left(2 \cosh ^{2} K\right)-\frac{1}{2} \sum_{\ell} \frac{\tau^{\ell}}{\ell}\left\langle\mathbf{0}\left|W^{*}(\ell)\right| \mathbf{0}\right\rangle=\ln \left(2 \cosh ^{2} K\right)-\frac{1}{2 N} \operatorname{tr}\left[\sum_{\ell} \frac{T^{* \ell} \tau^{\ell}}{\ell}\right] \\
& =\ln \left(2 \cosh ^{2} K\right)+\frac{1}{2 N} \operatorname{tr} \ln \left(1-\tau T^{*}\right) \\
& =\ln \left(2 \cosh ^{2} K\right)+\frac{1}{2 N} \sum_{\mathbf{q}} \operatorname{tr} \ln \left(1-\tau T^{*}(\mathbf{q})\right) .
\end{aligned}
$$

But for any matrix $M$, with eigenvalues $\left\{\lambda_{\alpha}\right\}$,

$$
\operatorname{tr} \ln M=\sum_{\alpha} \ln \lambda_{\alpha}=\ln \prod_{\alpha} \lambda_{\alpha}=\ln \operatorname{det} M
$$

Converting the sum over $\mathbf{q}$ in eq. (189) to an integral leads to

$$
\begin{aligned}
& \frac{\ln Z}{N}=\ln \left(2 \cosh ^{2} K\right)+ \\
& \quad \frac{1}{2} \int \frac{d^{2} \mathbf{q}}{(2 \pi)^{2}} \ln \left\{\operatorname{det}\left|\begin{array}{cccc}
1-\tau e^{-i q_{x}} & -\tau e^{-i\left(q_{x}-\frac{\pi}{4}\right)} & 0 & -\tau e^{-i\left(q_{x}+\frac{\pi}{4}\right)} \\
-\tau e^{-i\left(q_{y}+\frac{\pi}{4}\right)} & 1-\tau e^{-i q_{y}} & -\tau e^{-i\left(q_{y}-\frac{\pi}{4}\right)} & 0 \\
0 & -\tau e^{i\left(q_{x}-\frac{\pi}{4}\right)} & 1-\tau e^{i q_{x}} & -\tau e^{i\left(q_{x}+\frac{\pi}{4}\right)} \\
-\tau e^{i\left(q_{y}+\frac{\pi}{4}\right)} & 0 & -\tau e^{i\left(q_{y}-\frac{\pi}{4}\right)} & 1-\tau e^{i q_{y}}
\end{array}\right|\right\}
\end{aligned}
$$

Evaluation of the above determinant is straightforward, and the final result is

$$
\frac{\ln Z}{N}=\ln \left(2 \cosh ^{2} K\right)+\frac{1}{2} \int \frac{d^{2} \mathbf{q}}{(2 \pi)^{2}} \ln \left[\left(1+\tau^{2}\right)^{2}-2 \tau\left(1-\tau^{2}\right)\left(\cos q_{x}+\cos q_{y}\right)\right]
$$

Taking advantage of trigonometric identities, the result can be simplified to

$$
\frac{\ln Z}{N}=\ln 2+\frac{1}{2} \int_{-\pi}^{\pi} \frac{d q_{x} d q_{y}}{(2 \pi)^{2}} \ln \left[\cosh ^{2}(2 K)-\sinh (2 K)\left(\cos q_{x}+\cos q_{y}\right)\right] \quad .
$$

While it is possible to obtain a closed form expression by performing the integrals exactly, the final expression involves a hypergeometric function and is not any more illuminating. 


\section{R. Singular Behavior}

To uncover the singularity in the free energy of the two dimensional Ising model in eq. (191), we start with the simpler expression obtained by the unrestricted sum over random walks in eq. (172), (specializing to $d=2$ )

$$
f_{G}=\ln \left(2 \cosh ^{2} K\right)-\int \frac{d q_{x} d q_{y}}{(2 \pi)^{2}} \ln \left[1-2 \tau\left(\cos q_{x}+\cos q_{y}\right)\right] .
$$

Apart from the argument of the logarithm, this expression is similar to the exact result. Is it possible that such similar functional forms lead to distinct singular behaviors? The singularity results from the vanishing of the argument of the logarithm at $\tau_{c}=1 / 4$. In the vicinity of this point we make an expansion as in eq. (169),

$$
A_{G}(\tau, \mathbf{q})=(1-4 \tau)+\tau q^{2}+\mathcal{O}\left(q^{4}\right) \approx \tau_{c}\left(q^{2}+4 \frac{\delta \tau}{\tau_{c}}\right)
$$

where $\delta \tau=\tau_{c}-\tau$. The singular part of eq. (193) can be obtained by focusing on the behavior of the integrand as $\mathbf{q} \rightarrow \mathbf{0}$, and replacing the square Brillouin zone for the range of the integral with a circle of radius $\Lambda \approx 2 \pi$,

$$
\begin{aligned}
f_{\text {sing. }} & =-\int_{0}^{\Lambda} \frac{2 \pi q d q}{4 \pi^{2}} \ln \left(q^{2}+4 \frac{\delta \tau}{\tau_{c}}\right) \\
& =-\frac{1}{4 \pi}\left[\left(q^{2}+4 \frac{\delta \tau}{\tau_{c}}\right) \ln \left(\frac{q^{2}+4 \delta \tau / \tau_{c}}{e}\right)\right]_{0}^{\Lambda} .
\end{aligned}
$$

Only the expression evaluated at $q=0$ is singular, and

$$
f_{\text {sing. }}=-\frac{1}{\pi}\left(\frac{\delta \tau}{\tau_{c}}\right) \ln \left(\frac{\delta \tau}{\tau_{c}}\right)
$$

The resulting heat capacity $C_{G} \propto \partial^{2} f_{G} / \partial^{2} t$, diverges as $1 / \delta \tau$. Since eq. (193) is not valid for $\tau>\tau_{c}$, we cannot obtain the behavior of heat capacity on the low temperature side.

For the exact result of eq. (191), the argument of the logarithm is

$$
A^{*}(\tau, \mathbf{q})=\left(1+\tau^{2}\right)^{2}-2 \tau\left(1-\tau^{2}\right)\left(\cos q_{x}+\cos q_{y}\right) .
$$

The minimum value of this expression, for $\mathbf{q}=\mathbf{0}$, is

$$
A^{*}(\tau, \mathbf{0})=\left(1+\tau^{2}\right)^{2}-4 \tau\left(1-\tau^{2}\right)=\left(1-\tau^{2}\right)^{2}+4 \tau^{2}-4 \tau\left(1-\tau^{2}\right)=\left(1-\tau^{2}-2 \tau\right)^{2} .
$$


Since this expression (and hence the argument of the logarithm) is always non-negative, the integral exists for all values of $\tau$. As required, unlike eq. (193), the exact result is valid at all temperatures. There is a singularity when the argument vanishes for

$$
\tau_{c}^{2}+2 \tau_{c}-1=0 \quad \Longrightarrow \quad \tau_{c}=-1 \pm \sqrt{2} .
$$

The positive solution describes a ferromagnet and leads to a value of $K_{c}=\ln (\sqrt{2}+1) / 2$. Setting $\delta \tau=\tau-\tau_{c}$, and expanding eq. (197) in the vicinity of $\mathbf{q} \rightarrow 0$ gives

$$
\begin{aligned}
A^{*}(\tau, \mathbf{q}) & \approx\left[\left(-2 \tau_{c}-2\right) \delta \tau\right]^{2}+\tau_{c}\left(1-\tau_{c}^{2}\right) q^{2}+\cdots \\
& \approx 2 \tau_{c}^{2}\left[q^{2}+4\left(\frac{\delta \tau}{\tau_{c}}\right)^{2}\right] .
\end{aligned}
$$

The important difference from eq. (194) is that $\left(\delta \tau / \tau_{c}\right)$ appears at quadratic order. Following the steps in eqs. (195) and (196), the singular part of the free energy is

$$
\begin{aligned}
\left.\frac{\ln Z}{N}\right|_{\text {sing. }} & =\frac{1}{2} \int_{0}^{\Lambda} \frac{2 \pi q d q}{4 \pi^{2}} \ln \left[q^{2}+4\left(\frac{\delta \tau}{\tau_{c}}\right)^{2}\right] \\
& =\frac{1}{8 \pi}\left[\left(q^{2}+4\left(\frac{\delta \tau}{\tau_{c}}\right)^{2}\right) \ln \left(\frac{q^{2}+4\left(\delta \tau / \tau_{c}\right)^{2}}{e}\right)\right]_{0}^{\Lambda} \\
& =\frac{1}{\pi}\left(\frac{\delta \tau}{\tau_{c}}\right)^{2} \ln \left|\frac{\delta \tau}{\tau_{c}}\right| .
\end{aligned}
$$

The heat capacity is obtained by taking two derivatives and diverges as $C(\delta \tau)_{\text {sing. }}=$ $A_{ \pm} \ln |\delta \tau|$. The logarithmic singularity corresponds to the limit $\alpha=0$; the peak is symmetric and characterized by the amplitude ratio of $A_{+} / A_{-}=1$.

The graphical method presented in this section was originally developed by Kac and Ward [109]. The main ingredient of the derivation is the result that the correct accounting of the paths can be achieved by including a factor of $(-1)$ for each intersection. (This was originally a conjecture by Feynman [110], later proved by Sherman [111].) The change of sign is reminiscent of the exchange factor between fermions, and indeed the final result can be obtained by mappings to free fermions [112].

In addition to the partition function, the correlation functions $\left\langle\sigma_{\mathbf{i}} \sigma_{\mathbf{j}}\right\rangle$ can also be calculated by summing over paths [113]. Since the combination $q^{2}+4\left(\delta \tau / \tau_{c}\right)^{2}$ in eq. (200) describes the behavior of these random walks, we expect a correlation length $\xi \sim\left|\tau_{c} / \delta \tau\right|$, i.e. diverging with an exponent $\nu=1$ on both sides of the phase transition, with an amplitude ratio of unity. The exponents $\alpha$ and $\nu$ are related by the hyperscaling identity $\alpha=2-2 \nu$. The critical correlations at $\tau_{c}$ are more subtle and decay as $\left\langle\sigma_{\mathbf{i}} \sigma_{j}\right\rangle_{c} \sim$ $1 /|\mathbf{i}-\mathbf{j}|^{\eta}$ with $\eta=1 / 4$. Integrating the correlation functions yields the susceptibility, which diverges as $\chi_{ \pm} \simeq C_{ \pm}|\delta \tau|^{-\gamma}$, with $\gamma=7 / 4$ and $C_{+} / C_{-}=1$. 


\section{S. The Two Dimensional Spin Glass}

The key to the exact solution of the non-random two dimensional Ising model presented in the previous section is the reduction of the graphical expansion to sums over non-interacting (fermionic) random walks. This reduction depends on the geometrical properties of paths in $d=2$ and is independent of the uniformity of the bonds $J_{i j}$. The same method can be applied to the random bond problem, reducing the problem to (fermionic) random walks in a random medium. This is an undirected version of the problem of DPRM, extensively mentioned in these notes. Due to randomness, we can no longer diagonalize the transfer matrix that generates these walks by Fourier transformation. However, we can still examine such walks numerically by successive multiplications of the transfer matrix. Thus all random bond Ising models in $d=2$ can in principle be solved exactly in polynomial time in their size $L$. This is not true for the three dimensional versions.

We shall demonstrate the potentials of such exact methods by developing an algorithm for calculating the partition function of the $\pm J$ spin glass [51]. This is the model introduced in sec. I, where its high temperature correlation functions were examined with directed paths. The advantage of the model (or any other random mixture of $+J,-J$, and absent bonds) is that all computations can be performed in integer form, thus avoiding any floating point errors. Of course the main interest in the spin glass problem stems from the complexity of its low temperature states. Despite the great deal of work on spin glasses over the past decades [4, 114 [116], the description of the phase transition and the nature of the glassy state remain controversial subjects [117,118. Interactions with infinite-range [119] lead to a solution with broken replica symmetry [4. It is not known, however, to what extent this mean-field result captures the behavior of short-range interactions [118, 120]. Monte Carlo simulations of spin glass problems are notoriously difficult due the ease with which the system can get trapped in long-lived metastable states. It is thus quite valuable to provide some exact information about the equilibrium low temperature behavior of spin glasses, even if that knowledge is limited to two dimensions.

We start with the Edwards-Anderson (EA) Hamiltonian [121]

$$
H=\sum_{\langle i j\rangle} J_{i j} \sigma_{i} \sigma_{j},
$$


where the nearest neighbor quenched random bonds $J_{i j}$ are chosen from the bimodal distribution

$$
p\left(J_{i j}\right)=\frac{1}{2} \delta\left(J_{i j}-J\right)+\frac{1}{2} \delta\left(J_{i j}+J\right),
$$

with $J>0$. On a lattice with periodic boundary conditions (BCs), there are exactly $2 N$ bonds, with $N=L^{2}$ the total number of spins. The high temperature expansion for the partition function takes the form

$$
Z=2^{N} \cosh ^{2 N} K \sum_{\ell=0}^{2 N} A_{\ell} \tau^{\ell}
$$

where the coefficients $A_{l}$ are pure integers. Note that $A_{\ell}=0$ for odd values of $\ell$ since closed loops on the square lattice necessarily traverse an even number of bonds.

We can use the diagrammatic method introduced in the previous sections to transform the problem of summing the high temperature series into one of evaluating a local random walk. Every step proceeds exactly as before up to eq. (162). However, the $4 N \times 4 N$ transfer matrix in eq. (185) has to be modified to take care of the randomness in bonds. Each element of the matrix that connects sites $i$ and $j$ has to be multiplied with the reduced bond variable $s_{i j}=s_{j i}=J_{i j} / J$, equal to +1 for ferromagnetic bonds and -1 for antiferromagnetic bonds. There is an additional complication in dealing with finite lattice size. For simplicity we shall use periodic boundary conditions on an $L \times L$ lattice. We must then take proper account of diagrams which wrap around the lattice. The correct result, based on the combinatorics of closed loops on periodic lattices 122 is $Z=\left(-Z_{1}+\right.$ $\left.Z_{2}+Z_{3}+Z_{4}\right) / 2$ with

$$
Z_{\lambda}=2^{N} \cosh ^{2 N} K \sqrt{\operatorname{det}\left[1-T_{\lambda}^{*} \tau\right]}
$$

Here, $T_{1}^{*}$ is the original $4 N \times 4 N$ transfer matrix, while $T_{2}^{*}, T_{3}^{*}$, (and $T_{4}^{*}$ ) are obtained respectively by changing the sign of a horizontal, vertical, (or both) column of bonds. The linear combination $\left(-Z_{1}+Z_{2}+Z_{3}+Z_{4}\right) / 2$ ensures that all diagrams, including those which loop the entire lattice, are weighted correctly in the final expression for the partition function.

We have implemented [123] this algorithm on the computer as follows. Given a set of bonds $\left\{J_{i j}\right\}$, we first construct the $4 N \times 4 N$ matrices $T_{\lambda}^{*}$ and compute the traces $\operatorname{tr}\left(T_{\lambda}^{* \ell}\right)$ for $\ell \leq N$. This step of the algorithm is the most computationally intensive. The coefficients of the series expansions for $\ln Z_{\lambda}$ are related to the traces by

$$
\frac{\ln Z}{N}=\ln \left[2 \cosh ^{2} K\right]-\frac{1}{2 N} \sum_{\ell=0}^{\infty} \frac{1}{\ell} \operatorname{tr}\left(T^{* \ell}\right) \tau^{\ell} .
$$


Next, we compute the high temperature series for $Z$. This is done by exponentiating the series for $\ln Z_{\lambda}$, followed by taking the linear combination that incorporates periodic boundary conditions. The high temperature expansion for $Z$ is a polynomial in $\tau$ with integer coefficients; the last term, of order $2 N$, is derived from the graph that traverses every bond on the square lattice. These $2 N$ coefficients have an end-to-end symmetry that enables one to compute them from the first $N$ powers of the transfer matrix. Finally, we expand powers of $\cosh K$ and $\tanh K$ and rewrite $Z$ as a polynomial in $e^{-\beta J}$; the end result $Z=\sum_{E} g(E) e^{-\beta E}$ yields the density of states. For an Ising model with $\pm J$ bonds, we can perform all these operations using only integer arithmetic.

The algorithm has several desirable features. First, it returns the partition function $Z$ as an exact integer result. In this way, it not only avoids the statistical uncertainties inherent in Monte Carlo simulation; it also avoids the floating point errors that creep into numerically "exact" calculations of $Z$ in large systems at low temperatures. The algorithm thus provides us with an efficient and reliable way to investigate energies and entropies at low temperatures. This is particularly important in a system that exhibits a phase transition at $T=0$, such as the $\pm J$ spin glass. We can also calculate other quantities, such as the roots of the partition function in the complex plane, or the number of lowlevel excitations, that are otherwise inaccessible. Unfortunately, the necessity of handling large integers (of order $2^{N}$ ) complicates what would otherwise be a rather straightforward algorithm to implement.

A second advantage of the algorithm is that it executes in polynomial time. We estimate the algorithm's performance as follows. Computing the traces requires $O\left(N^{3}\right)$ arithmetic operations on integers of order $2^{N}$, while in general, the power series manipulations take much less time. We therefore expect the computation time to scale as $\tau \sim N^{\delta}$, with $3<\delta<4$. This stands in contrast to the numerical column to column transfer matrix method of Morgenstern and Binder [124] which has time and memory requirements that grow exponentially with system size. We obtained most of our results on dedicated DEC 3100 workstations. The largest lattice that we examined had $N=36 \times 36$ spins. Finally, we mention that the computation of the traces can be broken down into $O(N)$ independent computations, so that a faster, parallel implementation of the algorithm (on a supercomputer or spread across several workstations) should be possible. 


\section{T. Results for the Two Dimensional Spin Glass}

We examined the $\pm J$ spin glass on lattices of size $L=4$ to $L=36$. Several realizations of randomness were studied: 8000 for $L=4,6,8 ; 2000$ for $L=10,12,14 ; 800$ for $L=16$, 18; 80 for $L=20,22,24$; and 4 for $L=32,36$. We performed quenched averages by assigning an equal probability to each random sample: $\bar{\theta}=(1 / S) \sum_{s} \theta_{s}$. To reduce the amount of statistical error, we only considered lattices in which exactly half the plaquettes were frustrated [124]. We also found it quite revealing to compare our results with those for the fully frustrated Ising model [125, 126], as both models undergo phase transitions at $T=0$. The typical output of our algorithm is a set of integers $g(E)$ for the number of states. Using the density of states we can perform calculations in either the microcanonical or canonical ensemble. In the limit of infinite size, of course, the two ensembles should yield identical results.

We used the algorithm first to study the thermodynamic properties of the ground state, fitting the data for the ground-state energy and entropy to the finite-size form $f_{L}=f_{\infty}+a / L^{2}$. Extrapolating to infinite lattice size, we estimate $\overline{e_{0}} / J=-1.404 \pm 0.002$ and $\overline{s_{0}}=0.075 \pm 0.002$. These results are consistent with previous MC [127, 128] and column to column transfer matrix [129] estimates. We also used the algorithm to study the number of low-level excitations. On a lattice with periodic BCs, the lowest excited state has an energy $4 J$ above the ground state. The quantity $e^{\Delta S}=g\left(E_{0}+4 J\right) / g\left(E_{0}\right)$ measures the degeneracy ratio of these excited states. We find that $\overline{\Delta S}_{S G}$ grows faster than $\ln N$ indicating that the low-lying excitations of the $\pm J$ spin glass involve spin flips on large length scales.

The abundance of low-lying excitations affects the low-temperature behavior of the heat capacity. In a finite system with energy gap $4 J$, the heat capacity vanishes as $C \sim$ $\beta^{2} e^{-4 \beta J}$. As pointed out by Wang and Swendsen [127], this behavior can break down in the thermodynamic limit. The 1D Ising model with periodic BCs shows how this can happen: the energy gap is $4 J$, but the heat capacity of an infinite system vanishes as $C_{1 D} \sim \beta^{2} e^{-2 \beta J}$. The anomalous exponent reflects the fact that the number of lowest excited states grows as $N^{2}$. From Monte Carlo and column to column transfer matrix studies, Wang and Swendsen [127] conclude that $C_{S G} \sim \beta^{2} e^{-2 \beta J}$ for the $2 \mathrm{D} \pm J$ spin glass as well. Our results find a disagreement in slope between $\Delta S_{1 D}$ and $\overline{\Delta S}_{S G}$ versus $N$, leading us to suggest a different form for $C_{S G}$. As motivation, we appeal to another exactly soluble model with a phase transition at $T=0$ : the fully frustrated (FF) Ising 
model on a square lattice 125]. On a periodic lattice, the lowest excited states of the FF model have energy $4 J$ above the ground state. The large number of low-lying excitations, however, causes the heat capacity to vanish as $C_{F F} \sim \beta^{3} e^{-4 \beta J}$. Note the extra power of temperature. Our data compare much better to $\Delta S_{F F}$ than to $\Delta S_{1 D}$, suggesting a similar behavior may describe the $\pm J$ spin glass, e.g. $C_{S G} \sim \beta^{2+\rho} e^{-4 \beta J}$ with $\rho \neq 0$. As we shall see below, there are other reasons to favor this form.

One way to investigate phase transitions is to examine the roots of the partition function $Z$ in the complex plane. This was first done by Fisher [130 in a study of the $2 \mathrm{D}$ Ising model with uniform $+J$ bonds. Fisher calculated the distribution of roots of the partition function in the complex $z=e^{-2 \beta J}$ plane. In the limit of infinite lattice size, he showed that the roots condense onto two circles centered at $z= \pm 1$, and related the singular behavior in the free energy to the distribution of roots in the vicinity of the positive real axis. Since a system of finite size does not exhibit non-analytic behavior, it is clear that the roots of the partition function can only close in on the positive real axis in the thermodynamic limit. The zeros of partition functions are thus subject to finite-size scaling [131]. At a finite-temperature phase transition, the complex zero $T(L)$ closest to the positive real axis obeys $\left|T(L)-T_{c}\right| \sim L^{-y_{t}}$; likewise, the correlation length diverges as $\xi \sim\left(T-T_{c}\right)^{-\nu}$ with $\nu=1 / y_{t}$. On the other hand, at a $T=0$ phase transition, such as occurs in the 1D Ising model, one finds $|z(L)| \sim L^{-y_{z}}$ with $\xi \sim z^{-1 / y_{z}}$, where $z(L)$ is the smallest root in the complex $z=e^{-2 \beta J}$ plane. For example, the partition function of a 1D Ising chain with periodic boundary conditions in eq. (28),

$$
Z=2^{L} \cosh ^{L}(\beta J)\left[1+\tanh ^{L}(\beta J)\right]
$$

has its smallest root at $\tanh (\beta J)=e^{ \pm i \pi / L}$, or $z(L)= \pm i \tan (\pi / 2 L)$. As $L \rightarrow \infty$, the magnitude of the root scales as $z(L) \sim L^{-1}$, consistent with the fact that the correlation length in the $1 \mathrm{D}$ Ising model diverges as $\xi \sim e^{2 \beta J}$.

In the $\pm J$ spin glass, we observed that, for most realizations of randomness, the smallest root $z(L)$ falls on the imaginary axis. One might expect that the probability distribution for the magnitude of this root assumes a scale-invariant form as $L \rightarrow \infty$. We were unable to verify this hypothesis due to insufficient data on large lattices. Instead, we examined the statistics of $u(L)$, where $u=z^{2}=e^{-4 \beta J}$. On a square lattice with periodic boundary conditions, the partition function for a $\pm J$ spin glass is polynomial in $e^{-4 \beta J}$. We therefore looked at the scaling of roots in the complex $u=e^{-4 \beta J}$ plane. The 
results could be fitted to $|u(L)| \sim L^{-2.2}$ with $y_{u}=2.2 \pm 0.1$; this suggests to us that the correlation length in the $\pm J$ spin glass diverges as $\xi \sim e^{2 \beta J}$. Additional powers of temperature and/or finite-size effects might explain the slight deviation from $y_{u}=2$. Note that this behavior for the correlation length is consistent with the hyperscaling relation $\ln Z_{\text {singular }} \propto \xi^{-2}$, and our claim that, up to powers of temperature, the heat capacity diverges as $C \sim e^{-4 \beta J}$. This result disagrees with previous studies [127,129, 132, that report $\xi \sim T^{-\nu}$, with $\nu \approx 2.6-2.8$. An analytical approach, closely related to averaging the fermionic path integrals [133], does support exponential correlations, but with a gap of $4 J$.

A great deal of information on spin glasses has been obtained by examining 'defects' (droplets) in finite systems. The cost of a defect of size $L$ is related to the difference in free energies with periodic and anti-periodic BCs. At $T=0$, this reduces to the difference in energy between the ground states. On an $L \times L$ lattice, the defect energy measures the effective block coupling [134, 117, 118, 120, 1355 $J^{\prime}$ on length scale $L$. Let $p(L)$ be the fraction of $L \times L$ blocks for which $J^{\prime} \neq 0$. Scaling arguments [135] suggest that $p(L) \sim L^{-\eta}$, where $\eta$ is the critical exponent that characterizes the power law decay of correlations $\overline{\left.<\sigma_{0} \sigma_{L}\right\rangle^{2}}$ at $T=0$. Plotting $p(L)$ versus $L$, we find $\eta=0.22 \pm 0.06$ in agreement with previous results [135, 127]. Besides the defect energy, we also looked at the defect entropy $\delta S_{L}$, i.e. the difference in zero-temperature entropies with periodic and anti-periodic BCs. The data could be fitted to $\overline{\delta S_{L}^{2}} \sim L^{2 y_{S}}$ with $y_{S}=0.49 \pm 0.02$. This is curiously close to the result $\delta S_{L} \sim L^{1 / 2}$, expected if entropy changes due to reversing the different bonds along the boundary are statistically independent. The defect entropy in the fully frustrated Ising model approaches a constant value with $1 / L^{2}$ corrections. It is straightforward, moreover, to show that in the $1 \mathrm{D}$ Ising model the defect entropy scales as $\delta S_{L} \sim \ln L$. Both these behaviors are markedly different from the spin glass. We do not know any obvious relation between the finite-size scaling of the defect entropy and other quantities at $T=0$. More details of the algorithm and results can be found in ref. 123. 


\section{References}

[1] R. P. Feynman and A. R. Hibbs, Quantum Mechanics and Path Integrals (McGrawHill, New York, 1965).

[2] F. W. Wiegel, Introduction to Path-Integral Methods in Physics and Polymer Science (World Scientific, Singapore, 1986).

[3] N. I. Akhiezer, The Classical Moment Problem (Oliver and Boyd, London, 1965).

[4] M. Mézard, G. Parisi, and M. A. Virasoro, Spin Glass Theory and Beyond (World Scientific, Singapore, 1987).

[5] B. Z. Spivak, H. L. Zhao and S. Feng, preprint (1993).

[6] M. E. Fisher, J. Stat. Phys. 34, 667 (1984).

[7] M. Lässig, Phys. Rev. Lett. 73, 561 (1994).

[8] H. B. Thacker, Rev. Mod. Phys. 53, 253 (1981).

[9] M. Kardar, Nucl. Phys. B 290, 582 (1987).

[10] E. Medina and M. Kardar, J. Stat. Phys. 71, 967 (1992).

[11] W. Feller, An Introduction to Probability Theory and its Applications (Wiley, 1971).

[12] R. Friedberg and Y.-K. Yu, Phys. Rev. E 49, 4157 (1994).

[13] J.-P. Bouchaud and H. Orland, J. Stat. Phys. 61, 877 (1990).

[14] E. Medina and M. Kardar, Phys. Rev. B 46, 9984 (1992).

[15] D. A. Huse and C. L. Henley, Phys. Rev. Lett. 54, 2708 (1985).

[16] M. Kardar, Phys. Rev. Lett. 55, 2923 (1985).

[17] T. Halpin-Healy, Phys. Rev. A 45, 638 (1992); J. Krug, P. Meakin and T. HalpinHealy, Phys. Rev. A, 45638 (1992).

[18] J. M. Kim, M. A. Moore, and A. J. Bray, Phys. Rev. A 44, 2345 (1991).

[19] Y.-C. Zhang, Phys. Rev. Lett. 62, 979 (1989); Europhys. Lett. 9, 113 (1989); J. Stat. Phys. 57, 1123 (1989).

[20] A. Crisanti, G. Paladin, H.-J. Sommers, and A. Vulpiani, J. Phys. I (France) 2, 1325 (1992).

[21] G. Parisi, J. Physique (France) 51, 1595 (1990).

[22] M. Kardar and D. R. Nelson, Phys. Rev. Lett. 55, 1157 (1985).

[23] D. A. Huse, C. L. Henley, and D. S. Fisher, Phys. Rev. Lett. 55, 2924 (1985).

[24] M. Kardar and Y.-C. Zhang, Phys., Rev. Lett. 58, 2087 (1987).

[25] J. Imbrie and T. Spencer, J. Stat. Phys. 52, 609 (1988).

[26] Y. Y. Goldschmidt, Nucl. Phys. B 393, 507 (1993).

[27] D. S. Fisher and D. A. Huse, Phys. Rev. B 43, 10728 (1991).

[28] T. Halpin-Healy, Phys. Rev. Lett. 62, 442 (1989).

[29] L. Balents and D. S. Fisher, Phys. Rev. B 48, 5949 (1993).

[30] M. Mèzard and G. Parisi, J. Physique I 1, 809 (1991); J. Phys. A 23, L1229 (1990);

J. Physique I 2, 2231 (1992). 
[31] B. Derrida and H. Spohn, J. Stat. Phys. 51, 817 (1988).

[32] B. Derrida and R. B. Griffiths, Europhys. Lett. 8, 111 (1989); J. Cook and B. Derrida, J. Stat. Phys. 57, 89 (1989).

[33] See A. N. Berker S. Ostlund, J. Phys. C 12, 4961 (1979); and references therein.

[34] S. Roux, A. Hansen, L. R. di Silva, L. S. Lucena, and R. B. Pandey, J. Stat. Phys. 65, 183 (1991).

[35] E. Hopf, Comm. Pure Appl. Math. 3, 201 (1950); J. D. Cole, Quart. Appl. Math. 9, 225 (1951).

[36] M. Kardar, G. Parisi, and Y.-C. Zhang, Phys. Rev. Lett. 56, 889 (1986).

[37] D. Forster, D. R. Nelson, and M. J. Stephen, Phys. Rev. A 16, 732 (1977).

[38] E. Medina, T. Hwa, M. Kardar, and Y.-C. Zhang, Phys. Rev. A 39, 3053 (1989).

[39] T. Sun and M. Plischke, Phys. Rev. E 49, 5046 (1994).

[40] E. Frey and U. C. Täuber, Phys. Rev. E 50, 1024 (1994).

[41] See, e.g., Dynamics of Fractal Surfaces, edited by F. Family and T. Vicsek (World Scientific, Singapore, 1991); J. Krug and H. Spohn, in Solids Far From Equilibrium: Growth, Morphology and Defects, edited by C. Godreche (Cambridge University Press, Cambridge, 1991). A very enjoyable review article by T. Halpin-Healy and Y.-C. Zhang is currently under press; and a book by A. L. Barabasi and H. E. Stanley is also in preparation.

[42] B. M. Forrest and L.-H. Tang, Phys. Rev. Lett. 64, 1405 (1990).

[43] J. M. Kim, A. J. Bray, and M. A. Moore, Phys. Rev. A 44, 2345 (1991).

[44] M. A. Moore, T. Blum, J. P. Doherty, J.-P. Bouchaud, and P. Claudin, preprint (1994).

[45] See the contribution by S. Obukhov in Hopping Conduction in Semiconductors, by M. Pollak and B.I. Shklovskii (North Holland, 1990).

[46] V. L. Nguyen, B. Z. Spivak, and B. I. Shklovskii, Pis'ma Zh. Eksp. Teor. Fiz. 41, 35 (1985) [JETP Lett. 41, 42 (1985)]; Zh. Eksp. Teor. Fiz. 89, 11 (1985) [JETP Sov. Phys. 62, 1021 (1985)].

[47] Y. Shapir and X.-R. Wang, Europhys. Lett. 4, 1165 (1987).

[48] X.-R. Wang, Y. Shapir, E. Medina, and M. Kardar, Phys. Rev. B 42, 4559 (1990).

[49] J. Cook and B. Derrida, J. Stat. Phys. 61, 961 (1990).

[50] Y. Y. Goldschmidt and T. Blum, J. Physique I 2, 1607 (1992); T. Blum and Y. Y. Goldschmidt, J. Phys. A: Math. Gen. 25, 6517 (1992).

[51] G. Toulouse, Commun. Phys. 2, 115 (1977).

[52] W. H. Press, B.P. Flannery, S. A. Teukolsky, and W. T. Vetterling, Numerical Recipes, (Cambridge University Press 1986).

[53] Y.-C. Zhang, Phys. Rev. Lett. 62, 979 (1989); Europhys. Lett. 9, 113 (1989); J. Stat. Phys. 57, 1123 (1989).

[54] M. P. Gelfand, Physica A 177, 67 (1991).

[55] Y. Y. Goldschmidt and T. Blum, Nucl. Phys. B 380, 588 (1992). 
[56] Disorder and Fracture, edited by J. C. Charmet, S. Roux, and E. Guyon, (Plenum Press, New York, 1990).

[57] See, e.g., the article by H. J. Herrmann and L. de Arcangelis, in ref. [56]; and references therein.

[58] C. Poirier, M. Ammi, D. Bideau, and J.-P. Troadec, Phys. Rev. Lett. 68, 216 (1992).

[59] B. Kahng, G. G. Batrouni, S. Redner, L. de Arcangelis, and H. J. Herrmann, Phys. Rev. B 37, 7625 (1988).

[60] A. Hansen, E. L. Hinrichsen, and S. Roux, Phys. Rev. Lett. 66, 2476 (1991).

[61] P. A. Tydeman and A. M. Hiron, B. P. \& B.I.R.A. Bulletin 35, 9 (1964).

[62] J. Kertész, V. K. Horváth, and F. Weber, Fractals 1, 67 (1993).

[63] D. R. Nelson, Phys. Rev. Lett. 60, 1973 (1988); D. R. Nelson and H. S. Seung, Phys. Rev. B 39, 9153 (1989).

[64] See, for example, G. Blatter et. al., ETH preprint, and references therein.

[65] P. W. Anderson, Phys. Rev. 109, 1492 (1958).

[66] D. J. Thouless, Phys. Rep. 13, 93 (1974); Phys. Rev. Lett. 39, 1167 (1977).

[67] E. Abrahams, P. W. Anderson, D. C. Licciardello, and T. V. Ramakrishnan, Phys. Rev. Lett. 42, 673 (1979).

[68] L. P. Gor'kov, A. I. Larkin, and D. E. Khmel'nitskii, Zh. Eksp. Teor. Fiz. Pis'ma Red. 30, 248 (1979) [JETP Lett. 30, 248 (1979).].

[69] F. J. Wegner, Z. Phys. B 35, 207 (1979).

[70] P. A. Lee and T. V. Ramakrishnan, Rev. Mod. Phys. 57, 287 (1985).

[71] B. L. Altshuler, JEPT Lett. 41, 648 (1985).

[72] P. A. Lee and A. D. Stone Phys. Rev. Lett. 55, 1622 (1985).

[73] G. Bergmann, Phys. Rep. 107, 1 (1984).

[74] B. L. Altshuler and B. I. Shklovskii, Zh. Eksp. Teor. Fiz. 91, 220 (1986) [Sov. Phys. JETP 64, 127 (1986)].

[75] P. A. Lee, A. D. Stone, and H. Fukuyama, Phys. Rev. B 35, 1039 (1987).

[76] N. Zannon and J.-L. Pichard, J. Phys. (Paris) 49, 907 (1988).

[77] R. A. Webb and S. Washburn, Physics Today 41, No. 12, 46 (1988).

[78] P. A. Lee and B. L. Altshuler, Physics Today 41, No. 12, 36 (1988).

[79] N. F. Mott, J. Non-Cryst. Solids 1, 1 (1968).

[80] A. Miller and E. Abrahams, Phys. Rev. 120, 745 (1960).

[81] V. Ambegaokar, B. I. Halperin, and J. S. Langer, Phys. Rev. B 4, 2612 (1971).

[82] M. Pollak and B. I. Shklovskii, Hopping Conduction in Semiconductors (North Holland, 1990).

[83] O. Faran and Z. Ovadyahu, Phys. Rev. B38, 5457 (1988).

[84] K. Gottfried, Quantum Mechanics (Addison-Wesley, 1990).

[85] I. M. Lifshits and V. Ya. Kirpichenko, Sov. Phys. JETP 50, 499 (1979).

[86] E. Medina, M. Kardar, Y. Shapir, and X.-R. Wang, Phys. Rev. Lett. 62, 941 (1989). 
[87] E. Medina, M. Kardar, Y. Shapir, and X.-R. Wang, Phys. Rev. Lett. 64, 1816 (1990).

[88] E. Medina, and M. Kardar, Phys. Rev. Lett. 66, 3187 (1991).

[89] H. F. Jones, Groups, Representations and Physics (Adam Hilger, Bristol and New York, 1990).

[90] J.-P. Bouchaud, Europhys. Lett. 11, 505 (1990).

[91] A. A. Ovchinnikov and N. S. Erikhman, Sov. Phys. JETP 40, 733 (1975).

[92] A. M. Jayannavar and N. Kumar, Phys. Rev. Lett. 48, 553 (1982).

[93] L. Golubovic, S. Feng, and F. Zeng, Phys. Rev. Lett. 67, 2115 (1991).

[94] R. Dashen, J. Math Phys. 20, 894 (1979).

[95] H. de Raedt, A. Lagendijk, and P. de Vries, Phys. Rev. Lett. 62, 47 (1988).

[96] S. Feng, L. Golubovic, and Y.-C. Zhang, Phys. Rev. Lett. 65, 1028 (1990).

[97] R. Tao, Phys. Rev. A 43, 5284 (1991).

[98] For a review of localization of light, see S. John, Physics Today, 32 (May 1991).

[99] E. Medina, M. Kardar, and H. Spohn, Phys. Rev. Lett. 66, 2176 (1991).

[100] J.-P. Bouchaud, D. Touati, and D. Sornette, Phys. Rev. Lett. 68, 1787 (1992).

[101] L. Saul, M. Kardar, and N. Read, Phys. Rev. A 45, 8859 (1992).

[102] J. T. Chalker and P. D. Coddington, J. Phys. C21, 2665 (1988).

[103] C. Vanneste, P. Sebbah, and D. Sornette, Europhys. Lett. 17, 715 (1992).

[104] J.-P. Bouchaud and A. Georges, Phys. Rep. 195, 128 (1990).

[105] F. Igloi, Europhys. Lett. 16, 171 (1991).

[106] R. Friedberg and Y.-K. Yu, Phys. Rev. E 49, 5755 (1994).

[107] D. Cule and Y. Shapir, Phys. Rev. B 50, 5119 (1994).

[108] H. Whitney, Comp. Math. 4, 276 (1937).

[109] M. Kac and J. C. Ward, Phys. Rev. 88, 1332 (1952).

[110] R. P. Feynman, Statistical Mechanics (Addison-Wesley, Reading, MA, 1972).

[111] S. Sherman, J. Math. Phys. 1, 202 (1960).

[112] T. Schultz, D. Mattis, and E. Lieb, Rev. Mod. Phys. 36, 856 (1964).

[113] C. Itzykson and J.-M. Drouffe, Statistical field theory: 1 (Cambridge University Press, New York, 1989).

[114] K. Binder and A. P. Young, Rev. Mod. Phys. 58, 801 (1986).

[115] D. Chowdhury, Spin Glasses and Other Frustrated Systems (Princeton University Press, Princeton, NJ, 1986).

[116] K. H. Fischer and J. A. Hertz, Spin Glasses (Cambridge University Press, Cambridge, 1991).

[117] A. J. Bray and M. A. Moore, Phys. Rev. Lett. 58, 57 (1987).

[118] D. S. Fisher and D. A. Huse, Phys. Rev. B 38, 386 (1988).

[119] D. Sherrington and S. Kirkpatrick, Phys. Rev. Lett. 35, 192 (1975).

[120] S. R. McKay, A. N. Berker, and S. Kirkpatrick, Phys. Rev. Lett. 48, 767 (1982).

[121] S. F. Edwards and P. W. Anderson, J. Phys. F 5, 965 (1975). 
[122] R. B. Potts and J. C. Ward, Progr. Theoret. Phys. (Kyoto) 13, 38 (1955).

[123] L. Saul and M. Kardar, Phys. Rev. E 48, R3221 (1993); Nucl. Phys. B, in press (1994).

[124] I. Morgenstern and K. Binder, Phys. Rev. B 22, 288 (1980).

[125] J. Villain, J. Phys. C 10, 1717 (1977).

[126] G. Andre, R. Bidaux, J. P. Carten, R. Conte, and L. de Seze, J. Phys. (Paris) 40, 479 (1979).

[127] J.-S. Wang and R. H. Swendsen, Phys. Rev. B 38, 4840 (1988).

[128] B. A. Berg and T. Celik, Phys. Rev. Lett. 69, 2292 (1992).

[129] H.-F. Cheung and W. L. McMillan, J. Phys. C. 16, 7027 (1983).

[130] M. E. Fisher, Lectures in Theoretical Physics (University of Colorado Press, Boulder, 1964), Vol. 7c.

[131] C. Itzykson, R. B. Pearson, and J. B. Zuber, Nuclear Physics B220, 415 (1983).

[132] W. L. McMillan, J. Phys. C. 17, 3179 (1984).

[133] G. Jug, Phys. Rev. Lett. 53, 9 (1984); and private communications.

[134] W. L. McMillan, Phys. Rev. B 28, 5216 (1983).

[135] A. J. Bray and M. A. Moore, in Heidelberg Colloquium on Glassy Dynamics, edited by J. L. van Hemmen and I. Morgenstern (Springer-Verlag, Heidelberg, 1986). 